

\section{Near-Sun observations of an F-corona decrease and K-corona fine structure}

https://doi.org/10.1038/s41586-019-1807-x

Received: 28 June 2019

Accepted: 8 November 2019

Published online: 4 December 2019

\author{
R. A. Howard ${ }^{1 *}$, A. Vourlidas ${ }^{2}$, V. Bothmer ${ }^{3}$, R. C. Colaninno', C. E. DeForest ${ }^{4}$, B. Gallagher', \\ J. R. Hall ${ }^{5}$, P. Hess', A. K. Higginson' ${ }^{2}$, C. M. Korendyke', A. Kouloumvakos ${ }^{6}$, P. L. Lamy', \\ P. C. Liewer ${ }^{5}$, J. Linker ${ }^{8}$, M. Linton' ${ }^{1}$, P. Penteado ${ }^{5}$, S. P. Plunkett ${ }^{9}$, N. Poirier ${ }^{6}$, N. E. Raouafi ${ }^{2}$, \\ N. Rich', P. Rochus ${ }^{10}$, A. P. Rouillard ${ }^{6}$, D. G. Socker', G. Stenborg' ${ }^{1}$, A. F. Thernisien' \& N. M. Viall ${ }^{11}$
}

\begin{abstract}
Remote observations of the solar photospheric light scattered by electrons (the K-corona) and dust (the F-corona or zodiacal light) have been made from the ground during eclipses ${ }^{1}$ and from space at distances as small as 0.3 astronomical units ${ }^{2-5}$ to the Sun. Previous observations ${ }^{6-8}$ of dust scattering have not confirmed the existence of the theoretically predicted dust-free zone near the Sun ${ }^{9-11}$. The transient nature of the corona has been well characterized for large events, but questions still remain (for example, about the initiation of the corona ${ }^{12}$ and the production of solar energetic particles ${ }^{13}$ ) and for small events even its structure is uncertain ${ }^{14}$. Here we report imaging of the solar corona ${ }^{15}$ during the first two perihelion passes $(0.16-0.25$ astronomical units) of the Parker Solar Probe spacecraft ${ }^{13}$, each lasting ten days. The view from these distances is qualitatively similar to the historical views from ground and space, but there are some notable differences. At short elongations, we observe a decrease in the intensity of the F-coronal intensity, which is suggestive of the longsought dust free zone ${ }^{9-11}$. We also resolve the fine-scale plasma structure of very small eruptions, which are frequently ejected from the Sun. These take two forms: the frequently observed magnetic flux ropes ${ }^{12,16}$ and the predicted, but not yet observed, magnetic islands ${ }^{17,18}$ arising from the tearing-mode instability in the current sheet. Our observations of the coronal streamer evolution confirm the large-scale topology of the solar corona, but also reveal that, as recently predicted ${ }^{19}$, streamers are composed of yet smaller substreamers channelling continual density fluctuations at all visible scales.
\end{abstract}

The Parker Solar Probe (PSP) carries an imaging instrument, the Widefield Imager for Solar Probe (WISPR) ${ }^{15}$. The inset in Fig. 1a shows a WISPR inner telescope (WISPR-I) image taken on 6 November 2018 at the first perihelion. The Sun is $13.5^{\circ}$ to the left of the image and the width is about $40^{\circ}$. The locus of points at the apex of the contours defines the photometric axis of the F-corona. While most observations of the F-corona (or zodiacal light) have been taken from 1 astronomical unit (AU) away from the Sun, two spacecraft, Helios A and B, each carrying the Zodiacal Light Experiment ${ }^{5}$, orbited the Sun from 0.3 to $1.0 \mathrm{AU}$, one observing above the ecliptic plane and the other below. They measured the intensity / of the zodiacal light from varying heliocentric distances and found ${ }^{20}$ that it increases towards the Sun according to $/ \propto R_{\odot}{ }^{-n}$, where $n=2.3 \pm 0.1$ and $R_{\odot}$ is the radius of the Sun. The upper and lower limits were recorded at small and large elongations from the Sun, respectively, and were independent of the ecliptic longitude of the observer. The Sun Earth Connection Coronal and Heliospheric Investigation ${ }^{4}$ (SECCHI) heliospheric imagers $\mathrm{HI}-1^{21}$, onboard the STEREO spacecraft ${ }^{22}$ orbiting the Sun at approximately $1 \mathrm{AU}$, observed the corona at elongations ranging from 0.07 to $0.45 \mathrm{AU}\left(5^{\circ}-24^{\circ}\right)$ from the Sun. An analysis of intensities ${ }^{23}$ of the photometric axis of the F-corona from 2007 to 2014 found the exponent, for the entire elongation range covered by the HI-1 instrument, to be 2.31. Moreover, the analysis performed on restricted elongation ranges ${ }^{23}$ showed an identical tendency to the Helios results for the intensity gradient to increase towards the Sun $(n=2.29 \pm 0.10)$.

Figure 1a displays a log-log plot of a sample of F-coronal intensity profiles in units of mean solar brightness (MSB) along its photometric axis as measured by WISPR-I between $15^{\circ}$ and $50^{\circ}$ elongation from the centre of the Sun. The sample comprises data from five different heliocentric distances of the PSP spacecraft (0.336 AU to 0.166 AU) obtained during the orbit inbound to the first perihelion. Colour is used to distinguish the plots. For clarity we plot only these five positions, but all the profiles during the encounter are similar. These five profiles are normalized to the maximum intensity at $30^{\circ}$ elongation

${ }^{1}$ US Naval Research Laboratory, Washington, DC, USA. ${ }^{2}$ Johns Hopkins University, Applied Physics Laboratory, Laurel, MD, USA. ${ }^{3}$ Institut für Astrophysik, University of Göttingen, Göttingen,

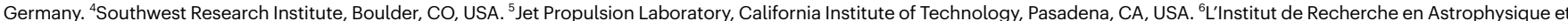
Planétologie, Toulouse, France. ${ }^{7}$ Laboratoire Atmosphères, Milieux et Observations Spatiales, CNRS and UVSQY, Guyancourt, France. ${ }^{8}$ Predictive Science Inc, San Diego, CA, USA. ${ }^{9}$ National

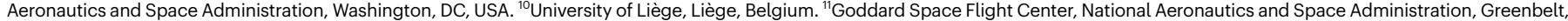
MD, USA. *e-mail: Russ.Howard@nrl.navy.mil 


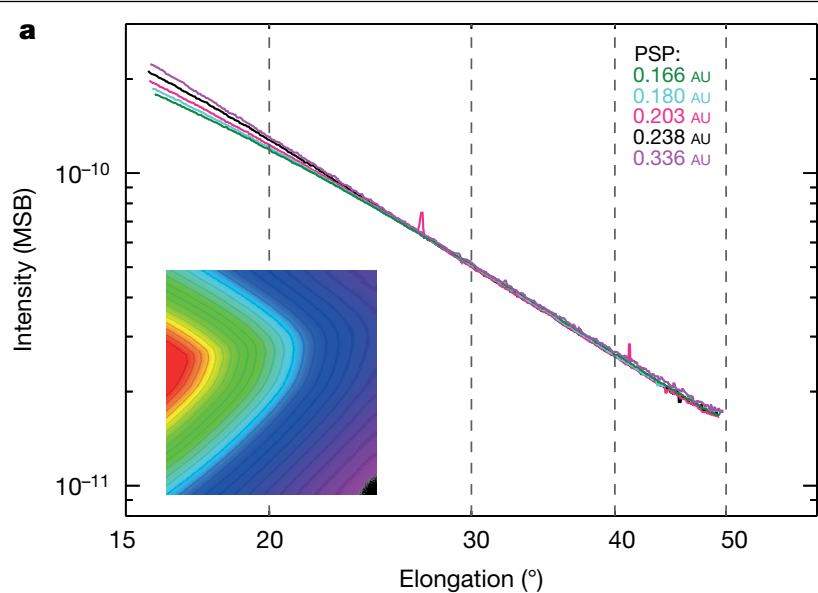

Fig. 1 | Intensity plots along the photometric axis of the F-corona. a, Observed intensities from WISPR-I for five heliocentric distances as a function of elongation (degrees) scaled to the same value at $30^{\circ}$ elongation.

to reveal the behaviour of the profiles for the various PSP heliocentric distances. Clearly, at larger elongations, the curves have exactly the same slope, and at shorter elongations $\left(<20^{\circ}\right)$, the intensity decreases with decreasing PSP distance, with the top plot (pink) for when PSP is the furthest from the Sun and the bottom plot (dark green) the closest. Figure $1 \mathrm{~b}$ shows the intensity profiles at the same five PSP distances for both telescopes, but plotted against elongations converted to $R_{\odot}$. The conversion to $R_{\odot}$ was performed by dividing the elongation by half the angular size of the Sun at the respective PSP distance. We note that the curves all overlie each other now, even the decreases seen in Fig. 1a. The small upward ticks are due to bright stars. The dashed blue line in Fig. $1 \mathrm{~b}$ shows the linear fit to the F-coronal intensities for elongations between $20 R_{\odot}$ and $77 R_{\odot}(n=2.31)$, a result identical to that obtained from both earlier observations ${ }^{20,23}$. For comparison, historical data ${ }^{3,24,25}$ have been added. The dashed green line depicts the linear fit to the LASCO-C3 data $^{3}$ (light green dots) for elongations greater than $13 R_{\odot}$, extrapolated down to $4 R_{\odot}$. The exponent, $n$, in this case is also

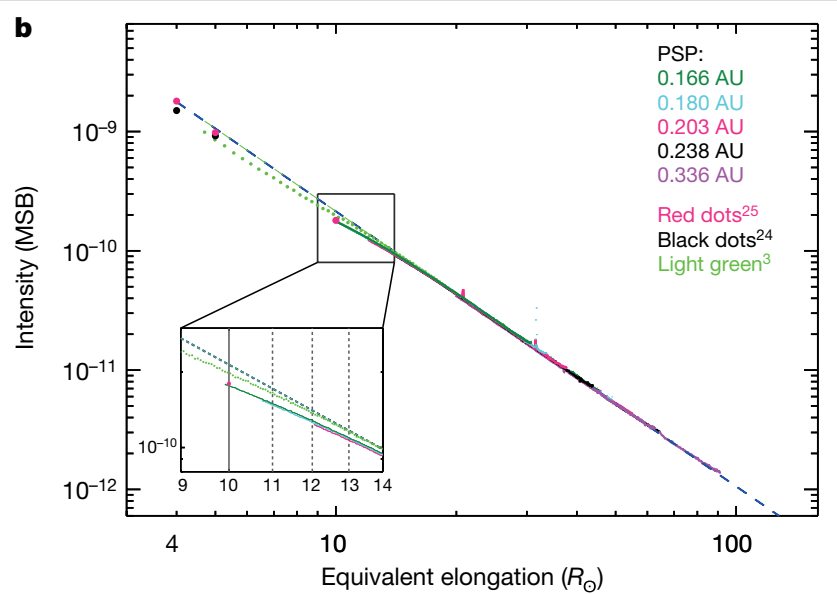

The inset in a shows an image of the F-corona taken on 6 November 2018. b, Observed intensities from both telescopes for five heliocentric distances as a function of elongation (solar radii). See text for further explanation.

2.31 (note the match between the blue and green dashed lines). The LASCO-C3 data were normalized to the WISPR value at $20 R_{\odot}$. For WISPR the absolute calibration was determined by analysing the intensity of stars in the field, which resulted in an error of $12 \%$. The relative accuracy and repeatability of the WISPR are excellent, which gives us high confidence in the turnover of the intensities below $17 R_{\odot}$. The historical measurements represented by the black dots ${ }^{24}$ and green dots ${ }^{3}$ both have absolute errors of $20 \%$. On the other hand, no error was given for the data represented by the red dots ${ }^{25}$.

Figure 2 shows the K-corona from both telescopes on 6 November 2018, after removal of the brighter background from the dust scattering. Supplementary Videos 1 and 2 provide background-removed videos of the images taken during the first two encounters. The grid lines for both Fig. 2 and the Supplementary Videos are in the HPLNARC, HPLT-ARC coordinate system ${ }^{26,27}$. The videos show the evolution of a coronal streamer during the two encounter periods of PSP observations. On the large scale, the agreement with model predictions

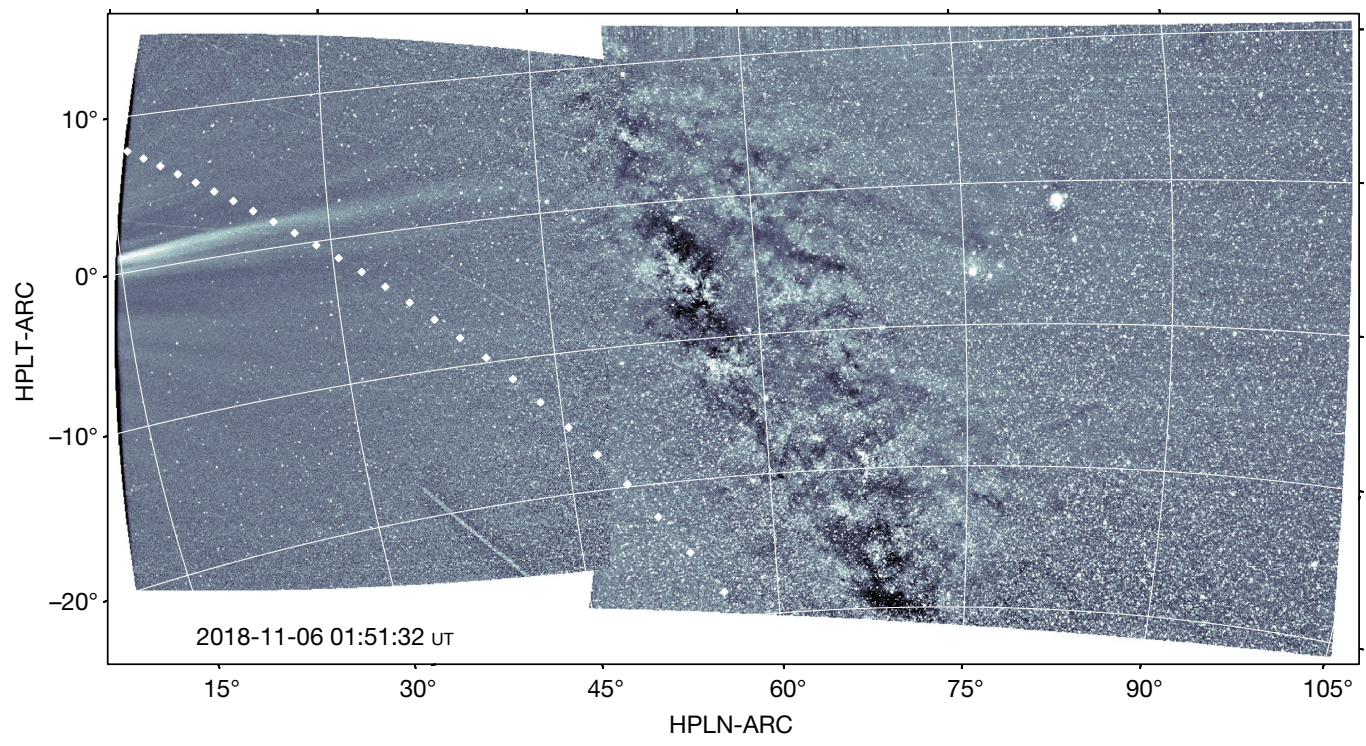

Fig. 2 Combined images from the inner and outer telescopes of WISPR on 6 November 2018 at 01:44 UT. After removal of an empirical model of the F-corona, the faint solar wind structures are revealed. A faint streamer outlining the heliospheric current sheet is visible, as are faint, radial and diffuse rays, all with apparent origin on the Sun. The image also reveals the dust trail along the orbit of the asteroid 3200 Phaethon (delineated by the white dots). The Galaxy dominates the scene in the inner part of the outer telescope accompanied by two bright objects:Jupiter (to the upper right) and the star Antares (a little below to its left) in the Scorpius constellation. 


\section{Article}
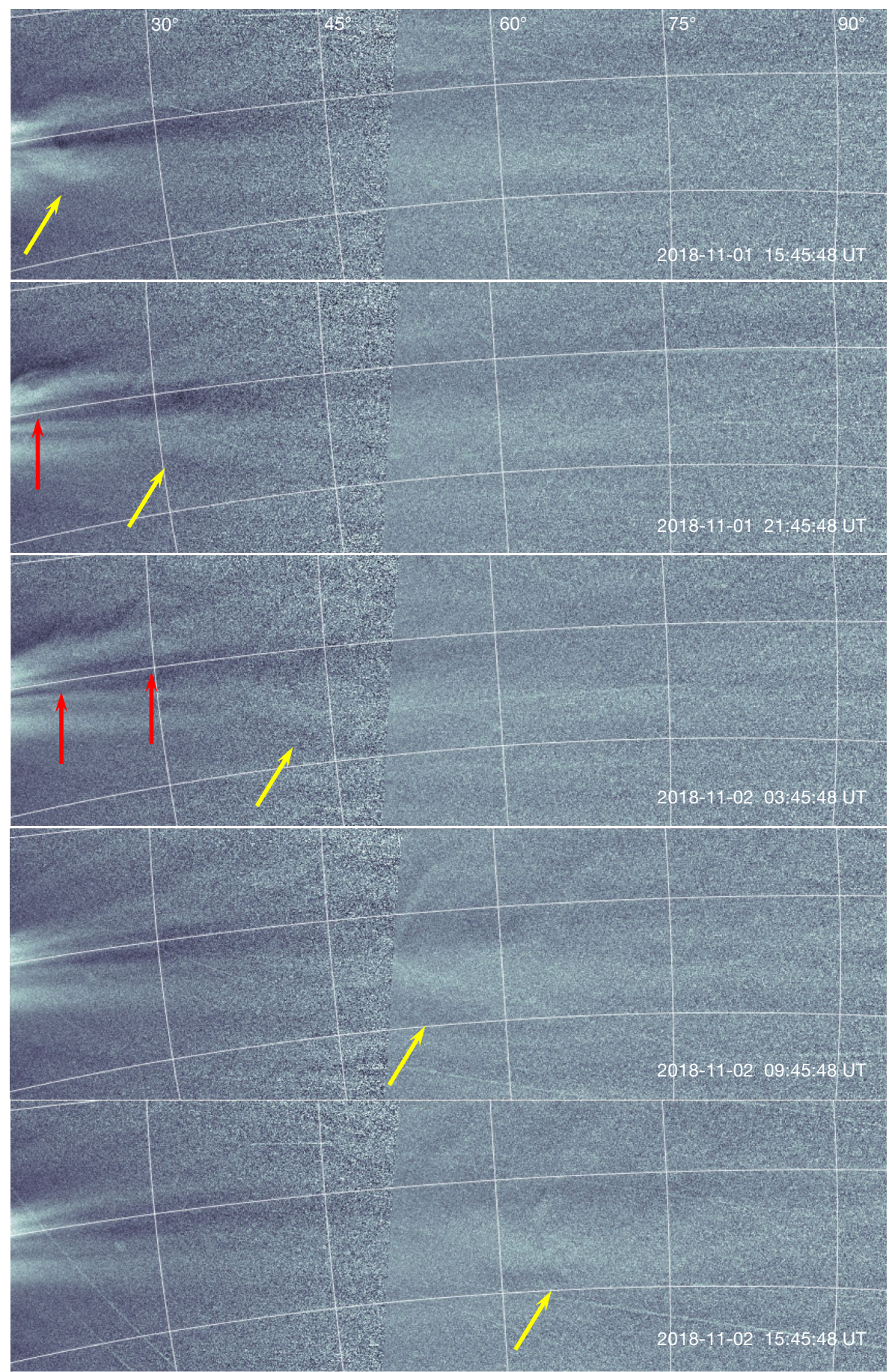

Fig. 3 | The propagation of a CME. Shown are five cropped frames from Supplementary Video 1 at different times in the same coordinate system as Fig. 2. The radial range is shown at the top, and the latitudinal range is $0^{\circ} \pm 10^{\circ}$ for each panel. The yellow and red arrows point to structures described in the text.

by our team (Extended Data Fig. 1) is very good, validating the model assumptions about the configuration of the magnetic field and the mass flux of the equatorial solar wind. The representation of WISPR-I images in a latitude versus time format (Extended Data Fig. 2a) reveals that near perihelion WISPR suddenly imaged faint coronal rays that are distinct from the main streamer rays. We note that fine structure along the streamer belt has been observed before ${ }^{19,28}$. High-resolution simulations of the corona reproduce these brightness features. We interpret their displacement to higher apparent latitudes to the spacecraft motion (Extended Data Fig. 2b). This striated 'texture' of the background corona is caused in our model by the spatial variability of coronal magnetic flux tubes, along which the plasma is heated and accelerated to form the slow solar wind.

Supplementary Video 1 shows a series of ejecta along the streamer. A particular event characterized by a big magnetic flux rope followed by several smaller ones is shown in Fig. 3. The first one (yellow arrows) has an elliptical high-density envelope surrounding a quasi-circular density depletion at its centre and a striated envelope. Although similar structures have been observed by LASCO, they could not always be resolved. The Encounter 1 images were binned $2 \times 2$ pixels, giving an effective 2-pixel spatial resolution ${ }^{15}$ of 60 arcsec (at $0.21 \mathrm{AU}$ ) for WISPR-I, which is about $2 \times$ finer than the LASCO-C 3 observations of this event. 

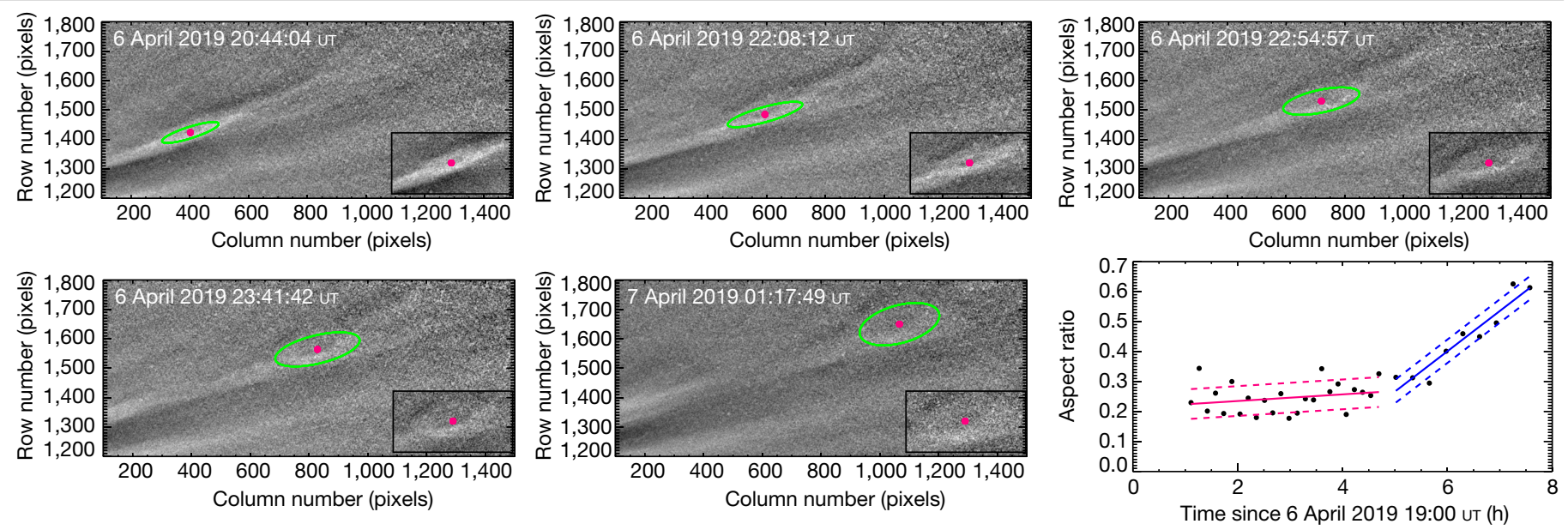

Fig. 4 | Formation and propagation of an island-like structure within a streamer. The first five panels show snapshots from WISPR-I during the second perihelion. An ellipse (green line) is fitted to the high-density ring of the structure in each panel. The final panel shows the aspect ratio (minor axis/ major axis) of these ellipse fits versus time. The red and blue solid lines show a linear fit to this aspect ratio from 20:00 UT to 23:45 UT on 6 April 2019 (red) and from 00:00 uT to 02:40 UT on 7 April 2019 (blue). The pairs of dashed lines on either side of these fits show the 1-sigma values.
On the other hand, LASCO-C2 tracked this structure when it was much closer to the Sun for a few images only, but with about $2.5 \times$ better spatial resolution. The event was also recorded by WISPR-O with an effective 2-pixel spatial resolution of 96 arcsec, extending the coverage of the event. Such density features have been interpreted as the boundaries of magnetic flux ropes ${ }^{12,16}$. We have combined the spatially resolved density information with modelling to locate the structures corresponding to the internal toroidal and poloidal magnetic fields and study their interactions with the ambient plasma as the structure expands inside the streamer rays. Preliminary work demonstrates (Extended Data Fig. 3) that the structures are indeed consistent with a force-free magnetic flux rope propagating along the heliospheric current sheet, which is quite flat during this period. The heliospheric current sheet flatness may be the reason for detecting the fine-scale structure of the event. Such behaviour is extremely rare in $1 \mathrm{AU}$ observations ${ }^{29}$.

The event shown in Fig. 3 (on 1 and 2 November 2018) also shows two additional smaller flux ropes (red arrows) following the northern boundary of the main flux rope; these are probably by-products of the interaction between the main event and the ambient corona. The quasi-circular shape and faint striations within the feature strongly suggest that it is an idealized magnetic flux rope. Smaller features, with similar morphologies, are also seen following the main ejection. The yellow arrows follow the first, main event. The red arrows follow the two following events, until they become merged with the background, although they are still visible in Supplementary Video 1. These structures were not detected by either LASCO or SECCHI, although both have observed many small ejecta ${ }^{14}$. Small dense features caused by interaction of a coronal mass ejection (CME) with its environment have proved difficult to identify positively ${ }^{30}$. Further studies will be necessary to test that idea.

Observations during the second perihelion (Supplementary Video 2) again show new dynamics in a coronal streamer. In this case, the observations capture the formation of oblong structures consistent with magnetic islands. Magnetic islands are, in two dimensions, a collection of roughly elliptical magnetic field lines that close on themselves; or, in three dimensions, helical field lines wrapping around a central (guide) field, again with a roughly elliptical cross-section. These island structures are predicted to form via the tearing-mode instability $^{17}$ from magnetic reconnection in a current sheet, such as the one within this streamer, where oppositely directed magnetic fields meet. Figure 4 shows several snapshots of this streamer and the formation and evolution of one of these oblong structures. This structure first appears at the inner edge of the image around 6 April 2019 20:00 UT and propagates out, within the current sheet, as an expanding, highly elliptical shape with a high-intensity (dense) ring of emission surrounding a low-intensity core. The final panel of Fig. 4 shows a measure of the aspect ratio (ratio of minor axis to major axis) of the ellipses fitted to this structure in each of the 33 frames from time 6 April 2019 19:57 UT to 7 April 2019 02:54 UT. Each ellipse was fitted to a set of points placed by hand on the high-density ring of the oblong structure in each frame. The corresponding ellipse is shown as a green curve in the five snapshots here, with a red dot at the centre of the ellipse. The plot indicates that the structure expands with a slightly increasing aspect ratio until 23:45 UT on 6 April 2019 and then it increases more quickly until the entire structure fades into the background. This evolution, including the increase in aspect ratio, is consistent with simulations of the tearing-mode formation of islands in an expanding coronal wind ${ }^{18}$. These simulations show the un-reconnected guide field collecting at the centre, forming this low-emission core, with the reconnecting field forming the high-density ring around the core. Although such an island ejection from a coronal streamer has been reported previously ${ }^{31}$, the earlier observations were not sufficiently resolved to show this internal ring and core structure.

WISPR imaged a variety of interesting structures in the corona/solar wind during the first two PSP orbits about the Sun. The departure from linearity of the F-corona intensity profiles below about $17 R_{\odot}$ is opposite to that found in both Helios and STEREO data. Although this behaviour could be leading to the predicted dust-free zone close to the Sun, the intensity decrease could be due to a change in the properties of the dust scattering, or a combination of the two. WISPR has certainly not observed the dust-free zone. Theoretical analyses of the plausible existence of a dust-free zone predict ${ }^{8-11}$ the formation of circumsolar dust bands that could be observed by their thermal emission. In a compilation of the 30 observations ${ }^{6}$ made at various wavelengths from $0.8 \mu \mathrm{m}$ to $3.6 \mu \mathrm{m}$ during eleven solar eclipses from 1966 through to 1998 , about half indicated an enhancement and the other half, including the two latest eclipses in 1991 and 1998, did not. The resolution of whether this WISPR finding represents dust depletion or something else will have to wait until PSP steps down to lower perihelia.

The near-corotation of PSP allows us to observe the radial outflow of the solar wind, without the confusing impact of solar rotation. The observations suggest that many small ejecta, commonly called 'blobs', may indeed be magnetic flux ropes but are usually too small to identify as such from $1 \mathrm{AU}\left(\right.$ ref. $^{32}$ ). Structures larger than these are generally 


\section{Article}

interpreted as CMEs, but the physical mechanism of formation may not be the same. This finding, particularly with the anticipated measurements of the same structures by PSP's in situ payload, may finally clarify the evolution of the CME magnetic structure in the heliosphere, opening up avenues of research on internal CME dynamics. As PSP steps closer to the Sun over the next five years, these observations, together with the modelling, will certainly provide insights and opportunities to study and separate the spatial and temporal variability of the solar wind near its source and will probably increase the performance of space weather prediction schemes. This will benefit a wide range of communities from basic physics research to space situational awareness to even astrophysics through exoplanet habitability applications.

\section{Online content}

Any methods, additional references, Nature Research reporting summaries, source data, extended data, supplementary information, acknowledgements, peer review information; details of author contributions and competing interests; and statements of data and code availability are available at https://doi.org/10.1038/s41586-019-1807-x.

1. Koutchmy, S. \& Lamy, P. L. The F-corona and the circum-solar dust evidences and properties. In IAU Colloq. 85: Properties and Interactions of Interplanetary Dust (eds Giese, R. H. \& Lamy, P.) (Reidel, 1985).

2. MacQueen, R. M. et al. The outer solar corona as observed from Skylab: preliminary results. Astrophys. J. 187, L85-L88 (1974).

3. Brueckner, G. et al. The large angle spectroscopic coronagraph (LASCO). Sol. Phys. 162, 357-402 (1995).

4. Howard, R. A. et al. Sun-Earth connection coronal and heliospheric investigation (SECCHI). Space Sci. Rev. 136, 67-115 (2008).

5. Leinert, C. \& Grun, E. Interplanetary dust. In Physics of the Inner Heliosphere Vol. I (eds Schwenn, R. \& Marsch, E.) (Springer, 1990).

6. Mann, I. et al. Dust near the Sun. Space Sci. Rev. 110, 269-305 (2004).

7. Leinert, C., Hanner, M., Link, H. \& Pitz, E. Search for a dust free zone around the Sun from the Helios 1 solar probe. Astron. Astrophys. 65, 119-122 (1978).

8. Lamy, P. et al. No evidence of a circumsolar dust ring from infrared observations of the 1991 solar eclipse. Science 257, 1377-1380 (1992).

9. Russell, H. N. On the composition of the Sun's atmosphere. Astrophys. J. 70, 11 (1929).

10. Lamy, P. L. The dynamics of circum-solar dust grains. Astron. Astrophys. 33, 191-194 (1974).
11. Mukai, T. \& Yamamoto, T. On the circumsolar grain materials. Publ. Astron. Soc. Jpn. 26, 445-458 (1979).

12. Vourlidas, A., Lynch, B. J., Howard, R. A. \& Li, Y. How many CMEs have flux ropes? Deciphering the signatures of shocks, flux ropes, and prominences in coronagraph observations of CMEs. Sol. Phys. 284, 179-201 (2013).

13. Fox, N. J. et al. The Solar Probe Plus mission: humanity's first visit to our star. Space Sci. Rev. 204, 7-48 (2016).

14. Sheeley, N. R. Jr, Lee, D. D.-H., Casto, K. P., Wang, Y.-M. \& Rich, N. B. The structure of streamer blobs. Astrophys. J. 722, 1522-1538 (2010).

15. Vourlidas, A. et al. The Wide-Field Imager for Solar Probe Plus (WISPR). Space Sci. Rev. 204, 83 (2016).

16. Chen, J. et al. Magnetic geometry and dynamics of the fast coronal mass ejection of 1997 September 9. Astrophys. J. 533, 481 (2000).

17. Furth, H. P., Killeen, J. \& Rosenbluth, M. N. Finite-resistivity instabilities of a sheet pinch. Phys. Fluids 6, 459 (1963).

18. Rappazzo, A. F., Velli, M., Einaudi, G. \& Dahlburg, R. B. Diamagnetic and expansion effects on the observable properties of the slow solar wind in a coronal streamer. Astrophys. J. 633, 474 (2005)

19. DeForest, C. E. et al. The highly structured outer solar corona. Astrophys. J. 862, 18 (2018).

20. Leinert, C., Richter, I., Pitz, E. \& Planck, B. The zodiacal light from 1.0 to 0.3 A.U. as observed by the HELIOS space probes. Astron. Astrophys. 103, 177-188 (1981).

21. Eyles, C. J. et al. The heliospheric imagers onboard the STEREO mission. Sol. Phys. 254, 387-445 (2009)

22. Kaiser, M. L. et al. The STEREO mission: an introduction. Space Sci. Rev. 136, 5-16 (2008),

23. Stenborg, G., Howard, R. A. \& Stauffer, J. R. Characterization of the white-light brightness of the F-corona between $5^{\circ}$ and $24^{\circ}$ elongation. Astrophys. J. 862, 168 (2018).

24. Saito, K., Poland, A. I. \& Munro, R. H. A study of the background corona near solar minimum. Sol. Phys. 55, 121-134 (1977).

25. Allen, C. W. Astrophysical Quantities (University of London, 1955)

26. Thompson, W. T. Coordinate systems for solar image data. Astron. Astrophys. 449, 791-803 (2006).

27. Calabretta, M. R. \& Greisen, E. W. Representations of celestial coordinates in FITS. Astron. Astrophys. 395, 1077-1122 (2002).

28. Thernisien, A. F. \& Howard, R. A. Electron density modeling of a streamer using LASCO data of 2004 January and February. Astrophys. J. 642, 523-532 (2006).

29. Vourlidas, A. et al. Comprehensive analysis of coronal mass ejection mass and energy properties over a full solar cycle. Astrophys. J. 694, 1471-1480 (2009).

30. Vourlidas, A., Maia, D., Pick, M. \& Howard, R. A. LASCO/Nancay observations of the CME on 20 April 1998: white light sources of type-II radio emission. In Magnetic Fields and Solar Prominences (ed. Wilson, A.) SP448, 1003 (European Space Agency, 1999).

31. Ko, Y.-K. et al. Dynamical and physical properties of a post-coronal mass ejection current sheet. Astrophys. J. 594, 1068-1084 (2003).

32. Rouillard, A. P. et al. The solar origin of small interplanetary transients. Astrophys. J. 734, 7 (2011).

Publisher's note Springer Nature remains neutral with regard to jurisdictional claims in published maps and institutional affiliations.

(c) The Author(s), under exclusive licence to Springer Nature Limited 2019 


\section{Methods}

WISPR contains two telescopes and measures the intensity of the visible light corona in addition to stellar and galactic sources. The two telescopes slightly overlap and have a combined field-of-view of $13.5^{\circ}$ to $108.5^{\circ}$ from the Sun, corresponding to approximately 9 to 78 solar $\operatorname{radii}\left(R_{\odot} ; 1 R_{\odot}=696,000 \mathrm{~km}\right)$ at $35.6 R_{\odot}$ perihelion. The visible light corona consists of two components: light scattered by free electrons (the K-corona) and light scattered by interplanetary dust (the F-corona). The F-corona of each WISPR image is removed using a technique ${ }^{36}$ similar to that developed for the SECCHI/HI-1. The primary difference is that for the HI-1 images the initial step in the procedure analysed the horizontal lines in the image, whereas here, the initial step uses the vertical lines in the images.

All of the data presented here have been calibrated into Mean Solar Brightness (MSB) units. The calibration details will be published in a future paper, but include the removal of geometric distortion, vignetting, instrumental artefacts (stray light, and so on) and then applying the photometric calibration of the system. The vignetting is caused by two sources: the projection of the image onto the two-dimensional plane of the Advanced Pixel Sensor detector and for WISPR-I the obscuration of the objective lens of the sunward side of the image by a series of baffles (including the PSP heat shield) which are used to block the solar disk illumination and block diffraction from the edges of the preceding baffles. The absolute calibration is confirmed on-orbit by measuring the intensity of stars passing through the field. The intensity of the stars as they transit across the image is also a check on the vignetting correction.

\section{Code availability}

The code used in the WISPR pipeline and analysis is available as part of the SolarSoft library (https://sohowww.nascom.nasa.gov/data/ software.html).

\section{Data availability}

The PSP Science Data Management Plan (https://sppgway.jhuapl.edu/ docs/data/7434-9101_Rev_A.pdf) requires that all science data from the first two orbits with calibrations must be released to the public within six months of downlink of the first orbit. In addition to this data type, we will be releasing background subtracted images, videos, and lists of events. Furthermore, the data must be delivered to the appropriate NASA/GSFC facility and integrated into the Virtual Observatory. Thus, the data is available from 12 November 2019. A complete archive is maintained at NRL (https://wispr.nrl.navy.mil) and will be publicly available at least during the full mission lifetime. A copy of the WISPR data will be located at the NASA/GSFC SDAC facility (https:// umbra.nascom.nasa.gov) and integrated into the Virtual Solar Observatory.

33. Pinto, R. \& Rouillard, A. P. A multiple flux-tube solar wind model. Astrophys. J. 838, 89 2017.

34. van Leeuwen, F. Validation of the new Hipparcos reduction. Astron. Astrophys. 474, 653-664 (2007)

35. Arge, C. N. et al. Air force data assimilative photospheric flux transport (ADAPT) model. In Twelfth International Solar Wind Conference (eds Maksimovic, M., Issautier, K., MeyerVernet, N., Moncuquet, M. \& Pantellini, F.) 343-346 (AIP, 2016).

36. Stenborg, G. \& Howard, R. A. A heuristic approach to remove the background intensity on white-light solar images. I. STEREO/HI-1 heliospheric images. Astrophys. J. 839, 68 (2017).

Acknowledgements We acknowledge the efforts of the PSP operations team in operating the mission and the WISPR team in developing and operating the instrument. We are grateful to R. Pinto (IRAP) for providing the Multi-VP magnetohydrodynamics simulations of the background solar wind used in Extended Data Fig. 3. R.A.H, A.V., R.C.C., C.E.DF., B.G., J.R.H., P.H., A.K.H., C.M.K., P.C.L., J.L., M.L., N.E.R., D.G.S., G.S. and A.F.T. acknowledge support from the NASA Parker Solar Probe Program Office. N.M.V. is supported through the NASA Heliophysics Internal Scientist Funding Model. A.P.R., A.K. and N.P. acknowledge financial support from the ERC for the project SLOW SOURCE - DLV-819189. P.L.L. acknowledges financial support from Centre National d'Etudes Spatiales. P.R. acknowledges support by the BELSPO/PRODEX. V.B. acknowledges the support of the Coronagraphic German and US Solar Probe Plus Survey (CGAUSS) project for WISPR by the German Aerospace Center (DLR) under grant 50OL1901 as a national contribution to the Parker Solar Probe mission.

Author contributions All authors contributed to writing the manuscript. R.A.H., A.V., N.R. and G.S. designed and collected the data. N.R., P.H., R.C.C., B.G. and G.S. performed the data processing and calibration. G.S. developed the technique for computing the background models. R.A.H., A.F.T., G.S. and P.L.L. performed the analysis of the dust scattering. A.V., C.E.DF., M.L., P.H., P.C.L., A.R., N.P., A.K., N.V., G.S., A.K.H., N.E.R., V.B., P.R. and R.A.H. carried out the analysis of the K-corona. J.L. assisted in the observation planning by providing magnetohydrodynamics model predictions. A.K.H. and N.E.R. coordinated the data acquisition and downlink. P.C.L., J.R.H. and P.P. assisted with data calibration, observation planning and analysis. R.A.H., N.R., A.V., P.L.L., S.P.P., C.M.K., R.C.C. and D.G.S. assisted with design, calibration and instrument checkouts.

Competing interests The authors declare no competing interests.

Additional information

Supplementary information is available for this paper at https://doi.org/10.1038/s41586-0191807-x.

Correspondence and requests for materials should be addressed to R.A.H. Peer review information Nature thanks Manuela Temmer and the other, anonymous, reviewer(s) for their contribution to the peer review of this work.

Reprints and permissions information is available at http://www.nature.com/reprints. 


\section{Article}

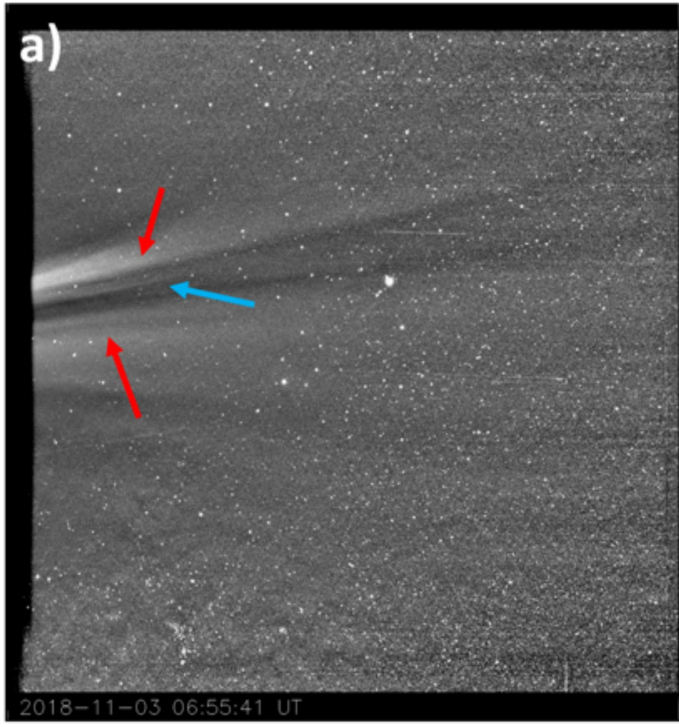

Extended Data Fig. 1 | Comparison of observations and synthetic

observations from magnetohydrodynamics model. a, An image from the inner WISPR telescope taken on 3 November 2018 at 06:55:41 UT. The field of view (of both panels) is $40^{\circ} \times 40^{\circ}$ with the Sun $13.5^{\circ}$ to the left. Two distinct sets of bright streamer rays are marked by red arrows. They are separated by a darker region marked by a blue arrow. The technique employed to remove the background F-corona in the WISPR image has artificially enhanced this dark region. The streamer rays located northwards of the dark region (top red arrow) are brighter than the rays situated southwards of the dark region (bottom red arrow). b, A synthetic white-light image produced from

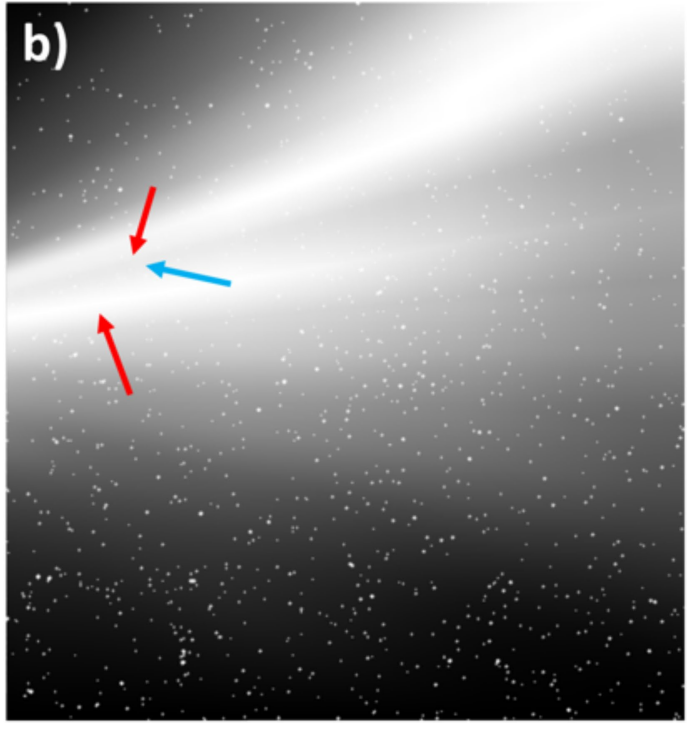

three-dimensional simulations of the solar wind by the MULTI-VP magnetohydrodynamics code using a Wilcox Solar Observatory photospheric magnetogram ${ }^{33}$. The three-dimensional density cubes produced by running the MULTI-VP code were processed by a white-light rendering code computing the brightness of the corona in the WISPR field of view from the heliocentric position of Parker Solar Probe. The MULTI-VP numerical model and the procedure to produce white-light images have been detailed ${ }^{33}$. The star field from the new Hipparchus astrometric catalogue ${ }^{34}$ was added to the simulated image in $\mathbf{b}$ for comparison with the WISPR image in $\mathbf{a}$. 
a) Observations

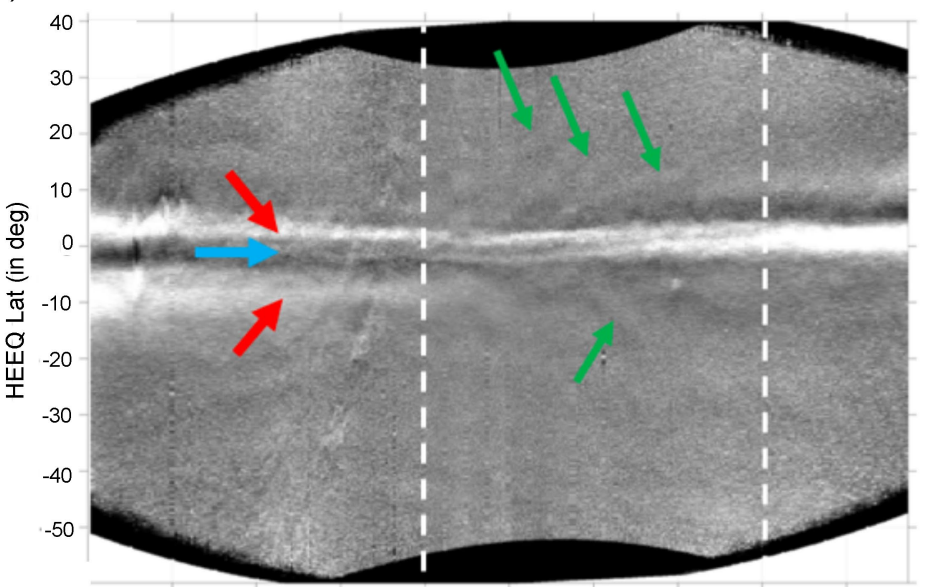

b) Model (low res.)

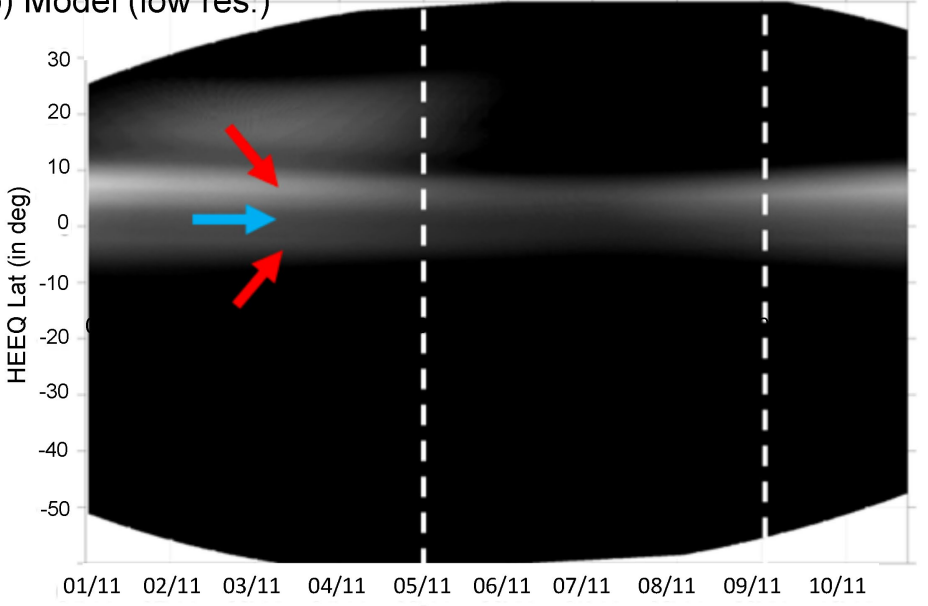

c) Model (high res.)

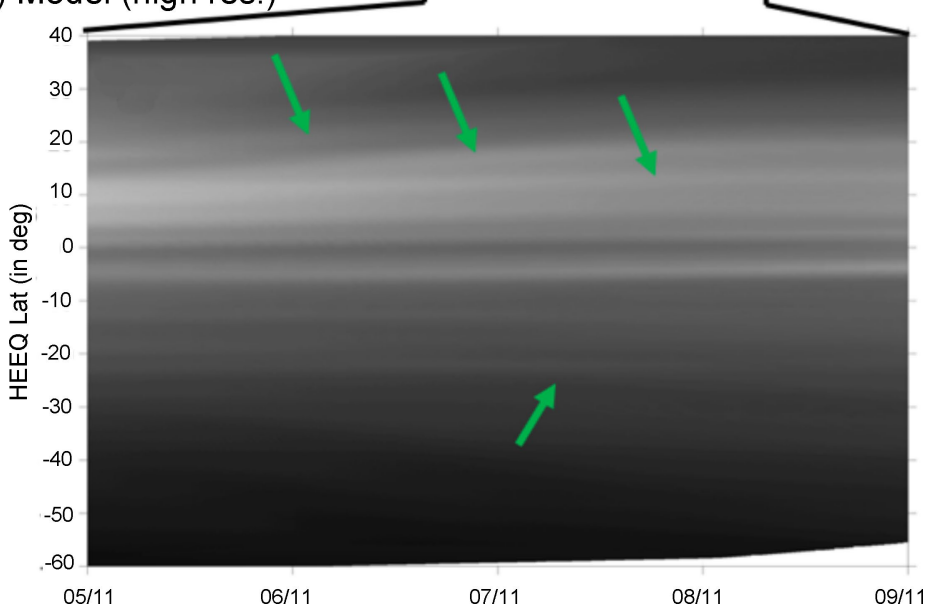

Extended Data Fig. 2 | Latitude versus time maps-observations and modelling. HEEQ, Heliocentric Earth Equator. a, A representation of WISPR inner telescope images in the form of a latitude versus time map. This map provides a summary of the temporal and spatial variability of coronal rays observed during the first encounter. We note that such fine structure along the streamer belt has been observed before ${ }^{19,28}$. We identify in these maps the main streamer rays already seen in Extended Data Fig. 1(the same blue and red arrows are shown here). During the period of super and corotation (5 to 9 November 2018), bright coronal rays drift in latitude away from the equator (green arrows). This is also visible in Supplementary Video 2.b, An equivalent map to a obtained from the WISPR synthetic images based on the MULTI-VP three-dimensional density cubes shown in Extended Data Fig. 1b. These medium-resolution simulations reproduce the time-varying aspect of the main streamer including their fading during perihelion (5 to 7 November). c, MULTI-VP high-resolution simulation results for the period 5 to 9 November 2018 based on 2-degree resolution magnetograms produced by the Air Force Data Assimilative Photospheric Flux (ADAPT) model ${ }^{35}$. The colour table has been saturated in these maps to enhance the features. The solar wind simulations reveal the finer striated structure of the corona and the coronal rays migrating poleward as observed by WISPR (green arrows). A search in the simulation data cubes reveals that these faint rays are separate from the brighter streamer rays. They form in the simulation as a result of considerable variability in the properties of the magnetic fields along which the slow solar wind forms. Since the prescribed coronal heating is scaled to the magnetic field properties this drives different mass flux along different flux tubes. We interpret the coronal rays marked by the top red arrows as resulting from the main streamer and the rays situated southwards (bottom red arrow) as resulting of a pseudo-streamer. 


\section{Article}

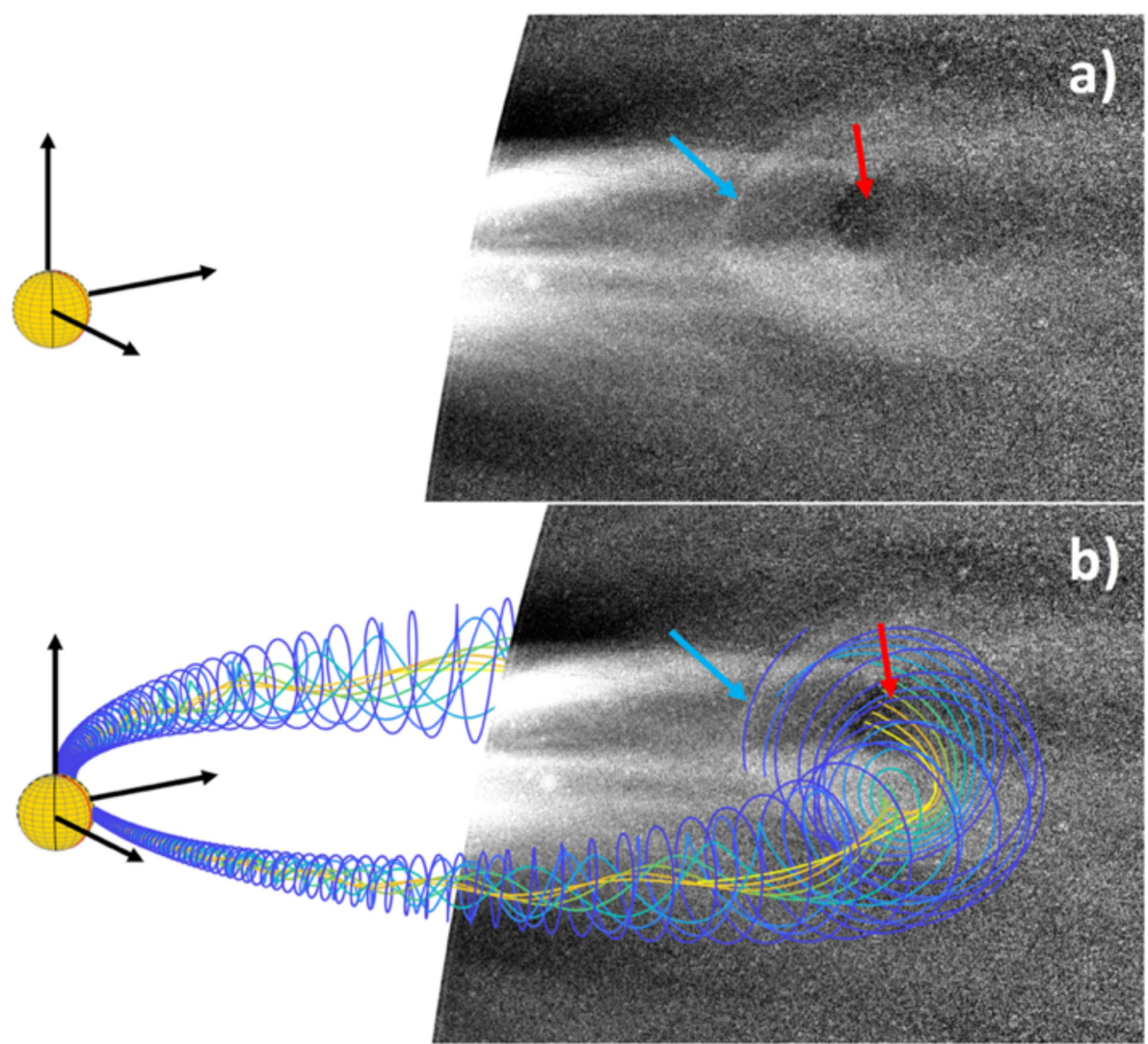

Extended Data Fig. 3 | Modelling of a CME as a 3D flux rope. a, An image from the inner WISPR telescope taken on 1 November 2018 at 19:30:50 UT during the passage of a pristine CME. Clear substructures are discernible in the WISPR image. The field of view is $40^{\circ} \times 40^{\circ}$ with the Sun $13.5^{\circ}$ to the left. A bright ring at the outer contour/boundary of the CME is indicated by a blue arrow. A striking feature of this CME event is the presence of a dark circular core located at the centre of the CME event and indicated by a red arrow.b. The same image as in a but with the results of a three-dimensional flux rope fit superimposed. This figure proposes an interpretation for the different features observed by WISPR based on our current understanding of the appearance of CMEs imaged in white light. The magnetic field lines (computed from solutions of the GradShafranov equation) of the CME are traced inside this flux rope. The bright ring (blue arrow) corresponds to plasma located on the boundary of the flux rope where the poloidal magnetic field lines of the CME are adjacent to the ambient solar wind plasma. The dark core (red arrow) marks the location where strong toroidal (axial) magnetic fields dominate the plasma locally. Detailed modelling of the event will be presented in a future dedicated publication. We acknowledge the use of the Wilcox Solar Magnetograms used in this paper, obtained from the website at http://wso.stanford.edu. 


\section{Probing the energetic particle environment near the Sun}

https://doi.org/10.1038/s41586-019-1811-1

Received: 28 June 2019

Accepted: 5 September 2019

Published online: 4 December 2019

\author{
D. J. McComas ${ }^{1 *}$, E. R. Christian ${ }^{2}$, C. M. S. Cohen ${ }^{3}$, A. C. Cummings ${ }^{3}$, A. J. Davis ${ }^{3}$, M. I. Desai ${ }^{4,5}$, \\ J. Giacalone ${ }^{6}$, M. E. Hill' ${ }^{7}$ C. J. Joyce', S. M. Krimigis ${ }^{7}$, A. W. Labrador ${ }^{3}$, R. A. Leske ${ }^{3}$, \\ O. Malandraki ${ }^{8}$, W. H. Matthaeus ${ }^{9}$, R. L. McNutt Jr ${ }^{7}$, R. A. Mewaldt ${ }^{3}$, D. G. Mitchell ${ }^{7}$, A. Posner ${ }^{10}$, \\ J. S. Rankin', E. C. Roelof ${ }^{7}$, N. A. Schwadron ${ }^{1,11}$, E. C. Stone ${ }^{3}$, J. R. Szalay', M. E. Wiedenbeck ${ }^{12}$, \\ S. D. Bale ${ }^{13,14}$, J. C. Kasper ${ }^{15}$, A. W. Case ${ }^{16}$, K. E. Korreck ${ }^{16}$, R. J. MacDowall' ${ }^{2}$, M. Pulupa ${ }^{13}$, \\ M. L. Stevens ${ }^{16}$ \& A. P. Rouillard ${ }^{17}$
}

\begin{abstract}
NASA's Parker Solar Probe mission ${ }^{1}$ recently plunged through the inner heliosphere of the Sun to its perihelia, about 24 million kilometres from the Sun. Previous studies farther from the Sun (performed mostly at a distance of 1 astronomical unit) indicate that solar energetic particles are accelerated from a few kiloelectronvolts up to nearrelativistic energies via at least two processes: 'impulsive' events, which are usually associated with magnetic reconnection in solar flares and are typically enriched in electrons, helium- 3 and heavier ions ${ }^{2}$, and 'gradual' events ${ }^{3,4}$, which are typically associated with large coronal-mass-ejection-driven shocks and compressions moving through the corona and inner solar wind and are the dominant source of protons with energies between 1 and 10 megaelectronvolts. However, some events show aspects of both processes and the electron-proton ratio is not bimodally distributed, as would be expected if there were only two possible processes 5 . These processes have been very difficult to resolve from prior observations, owing to the various transport effects that affect the energetic particle population en route to more distant spacecraft ${ }^{6}$. Here we report observations of the near-Sun energetic particle radiation environment over the first two orbits of the probe. We find a variety of energetic particle events accelerated both locally and remotely including by corotating interaction regions, impulsive events driven by acceleration near the Sun, and an event related to a coronal mass ejection. We provide direct observations of the energetic particle radiation environment in the region just above the corona of the Sun and directly explore the physics of particle acceleration and transport.
\end{abstract}

Onboard the Parker Solar Probe (PSP), the instrument suite of the Integrated Science Investigation of the Sun (IS॰IS) ${ }^{7}$ has made the first nearSun measurements of solar energetic particles (SEPs). IS $\odot$ IS comprises two energetic particle instruments with overlapping coverage, $\mathrm{EPI}-\mathrm{Hi}$ and EPI-Lo, measuring higher- and lower-energy particles ${ }^{7}$. Together they enable IS॰IS to explore the near-Sun environment by measuring the fluxes, energy spectra, anisotropy, and composition of suprathermal and energetic ions with energies from about 0.02 to $200 \mathrm{MeV}$ per nucleon and electrons with energies from about 0.05 to $6 \mathrm{MeV}$. Here we examine this energetic particle environment in the context of in situ solar wind ${ }^{8}$ and magnetic field ${ }^{9}$ conditions and surrounding density structure ${ }^{10}$ measured by other instruments onboard PSP.

Figure 1 summarizes IS $\odot I S$ observations of energetic particles over PSP's first two orbits. Higher-(1-2 MeV) and lower-energy (30-200 keV)
$\mathrm{H}^{+}$ion count rates are plotted on the outside and inside of the orbital trajectory, respectively. Intensifications indicate energetic particle events, with some seen only at higher energies, some only at lower energies, and others simultaneously across the combined energy range. Figure 1 indicates how rich the IS $\odot I S$ observations are: a broad array of different types of particle events are seen at all distances.

The first large intensification occurred during orbit 1 at higher energies with PSP inbound (during interval a, 2018-287 18:00 to 2018-297 08:20 universal time (UT)) at about 0.5 astronomical units (AU). Although not obvious from Fig. 1, this is a corotational event also seen when PSP was outbound at about $0.65 \mathrm{AU}$ (during interval b, 2018-330 23:20 to 2018-341 15:00 UT). Corotating interaction regions (CIRs) form as faster solar wind piles up behind slower wind, forming a compression ${ }^{11,12}$. Because these faster solar wind streams

${ }^{1}$ Department of Astrophysical Sciences, Princeton University, Princeton, NJ, USA. ${ }^{2}$ Goddard Space Flight Center, Greenbelt, MD, USA. ${ }^{3}$ California Institute of Technology, Pasadena, CA, USA. ${ }^{4}$ Southwest Research Institute, San Antonio, TX, USA. ${ }^{5}$ University of Texas at San Antonio, San Antonio, TX, USA. ${ }^{6}$ University of Arizona, Tucson, AZ, USA. ${ }^{7}$ Johns Hopkins University Applied Physics Laboratory, Laurel, MD, USA. ${ }^{8}$ National Observatory of Athens, IAASARS, Athens, Greece. ${ }^{~}$ University of Delaware, Newark, DE, USA. ${ }^{10} \mathrm{NASA}$ HQ, Washington, DC, USA. ${ }^{11}$ University of New Hampshire, Durham, NH, USA. ${ }^{12}$ Jet Propulsion Laboratory, California Institute of Technology, Pasadena, CA, USA. ${ }^{13}$ University of California at Berkeley, Berkeley, CA, USA. ${ }^{14}$ The Blackett Laboratory, Imperial College London, London, UK. ${ }^{15}$ University of Michigan, Ann Arbor, MI, USA. ${ }^{16}$ Smithsonian Astrophysical Observatory, Cambridge, MA, USA. ${ }^{17} \mathrm{CNRS}$, Toulouse, France. *e-mail: dmccomas@princeton.edu 


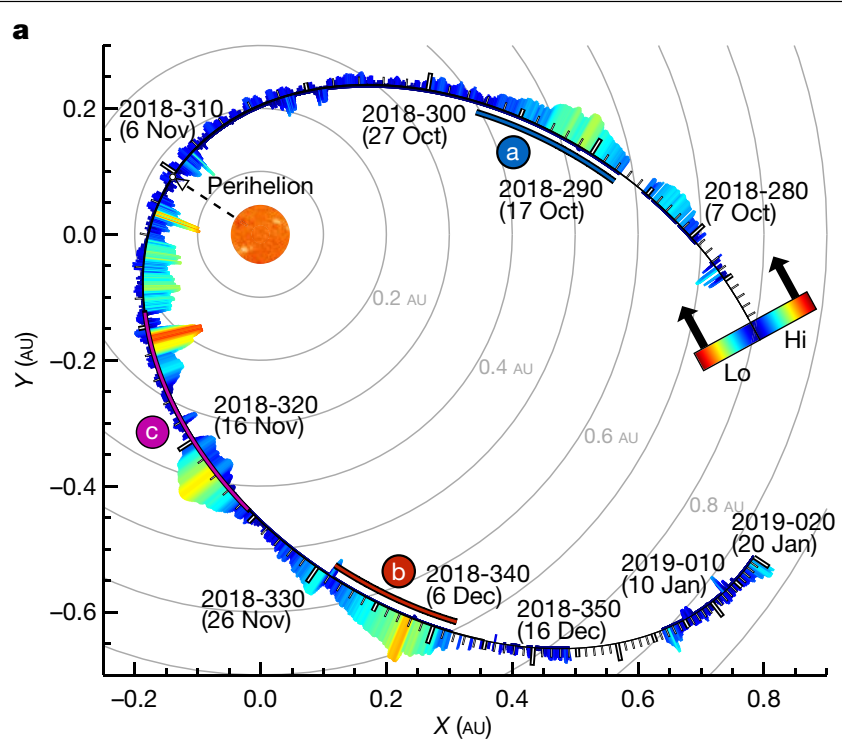

Fig. 1 Observations of energetic particles during orbits 1 and 2. a, b, Observations of energetic particles (primarily $\mathrm{H}^{+}$) at lower (Lo, about 30$200 \mathrm{keV}$; inside track) and higher energies (Hi, about 1-2 MeV; outside track) from PSP's orbit 1 (a) and orbit 2 (b). Intervals without data are indicated by the

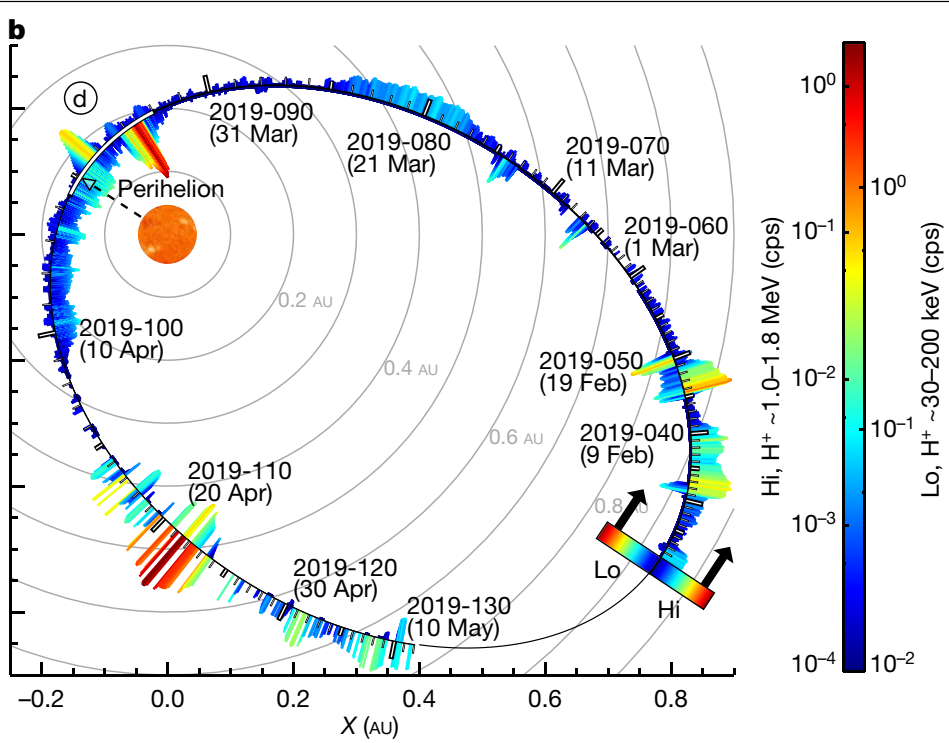

black orbital track. Particle intensity is indicated by both the colour and the length of the bars. Intervals a-d are earmarked for detailed study. The scale indicated by white rectangles on the outer track is measured in days UT, from 2018-280 (7 October 2018) to 2019-020 (20 January 2019). emanate from coronal holes at the Sun, CIRs map to nearly fixed solar longitudes.

Figure 2 shows intervals a and $b$ as a function of the longitude of the solar surface 'foot point' magnetically connected to the spacecraft, calculated for a nominal Parker spiral with a fixed solar wind speed of $350 \mathrm{~km} \mathrm{~s}^{-1}$. This calculation combines the rotation of the Sun and spacecraft location to show that both events arise from the same, single CIR structure. These events are 'dispersionless'-all ions arrive at roughly the same time and fluctuations in intensity are consistent across ion speeds. Such events indicate that PSP passed across magnetic flux tubes that were already filled with high-energy $(>1 \mathrm{MeV})$ particles that move quickly along magnetic field lines. The intensities of sunward- and antisunward-moving particles in intervals $a$ and b were similar (Fig. 2a), consistent with a corotating structure that traps particles between a
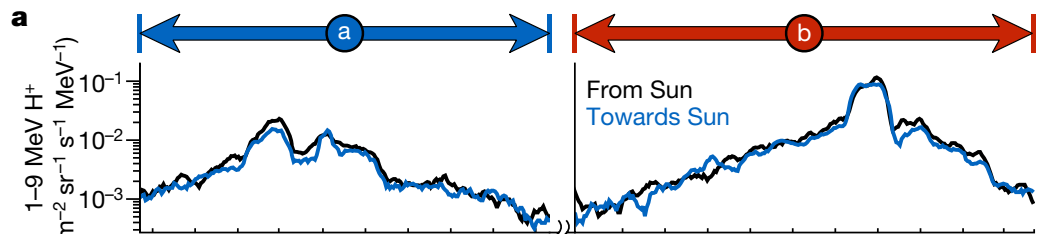

b

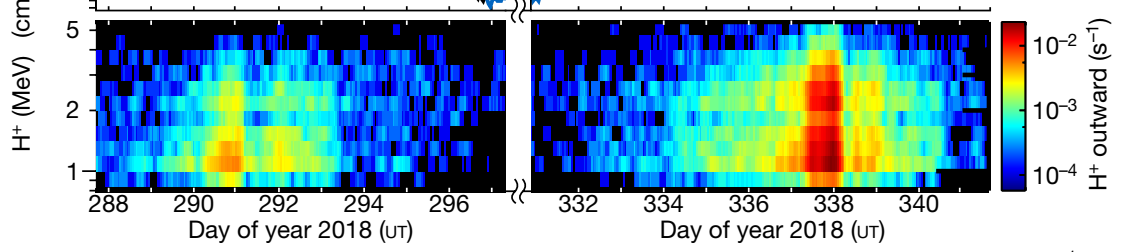

c

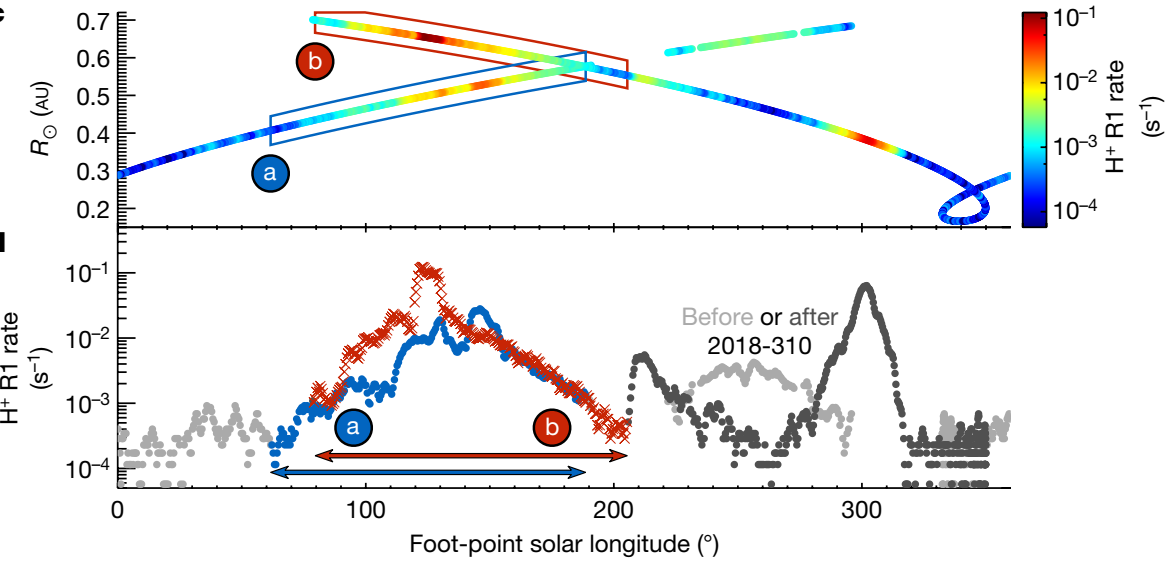

Fig. 2 Recurring corotating energetic particle events. a-d, Corotating ion events observed in intervals a (blue) and $b$ (red) represented by sunward/antisunward flux time series (a), a count-rate spectrogram (b), the flux as a function of the magnetic foot point in Carrington longitude and radius from the Sun (c), and the $1-2 \mathrm{MeV} \mathrm{H}^{+}$rate versus foot-point longitude (d). In c, d, $v_{\mathrm{sw}}=350 \mathrm{~km} \mathrm{~s}^{-1}$ is a nominal solar wind speed. 


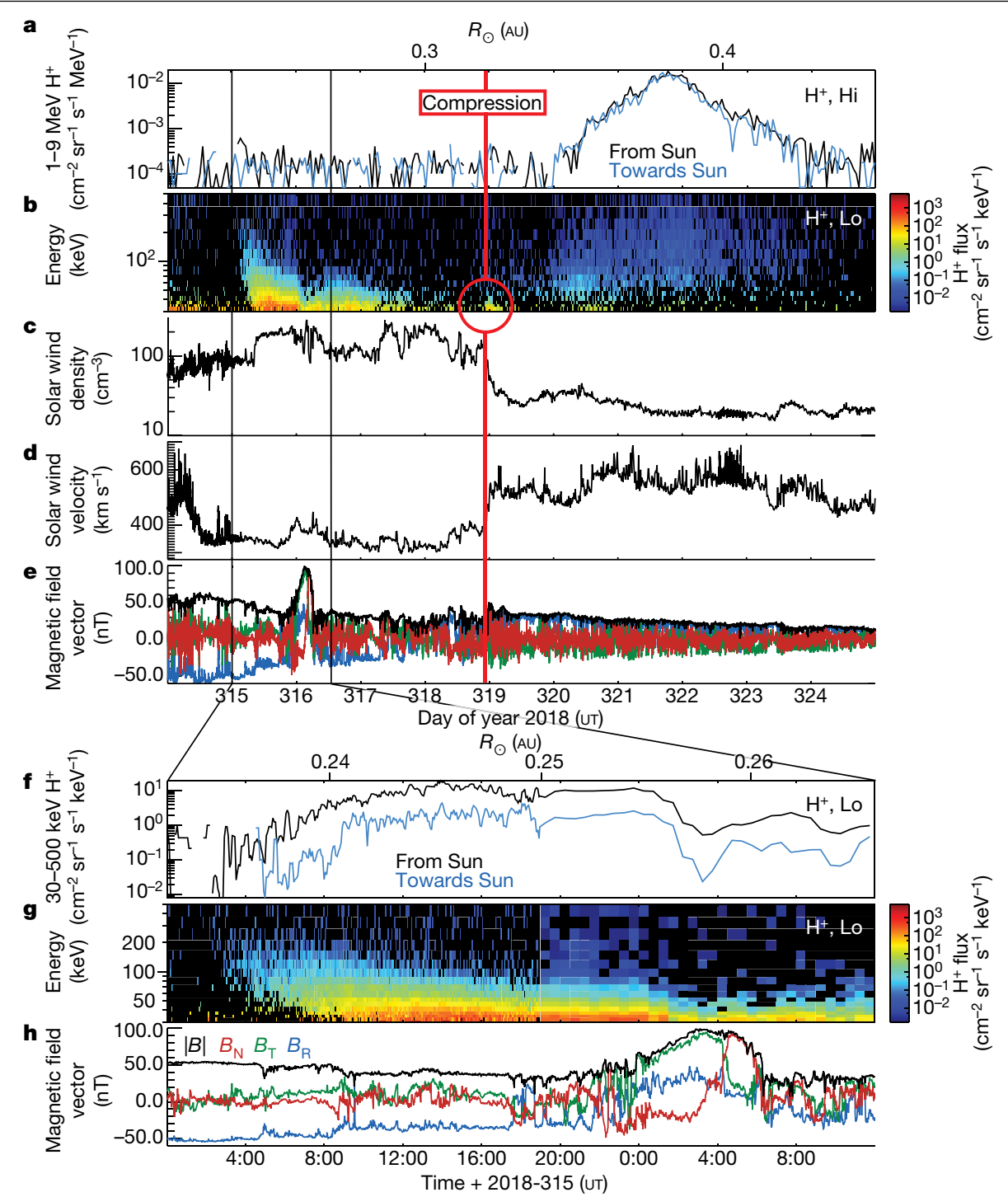

Fig. 3 |CME-related low-energy event and subsequent high-energy event. a-e, Time series of primarily proton flux at $>1 \mathrm{MeV}(\mathbf{a}), \mathrm{H}^{+}$flux around $30-$ $500 \mathrm{keV}(\mathbf{b})$, solar wind density (c) and radial speed ${ }^{13}(\mathbf{d})$, and magnetic field vector and magnitude ${ }^{14}(\mathbf{e})$ over interval c. $\mathbf{f}-\mathbf{h}$, Magnification of the dispersive SEP event and CME for $\mathrm{H}^{+}$flux around $30-500 \mathrm{keV}$. source more distant than the spacecraft and the increasing magnetic field strength closer to the Sun. The particle acceleration probably occurs at reverse shocks, which typically form beyond about $2 \mathrm{Au}$ from compressions in such CIRs.

The inbound leg towards perihelion 1 (which occurred at $0.17 \mathrm{AU}$, at 03:28 6 November $2018 \mathrm{UT}$ ) was extremely quiet from about $0.4 \mathrm{AU}$, providing an ideal opportunity for other PSP instruments ${ }^{13,14}$ to observe very quiet solar wind conditions with essentially no SEP-produced penetrating backgrounds. IS $\odot$ IS began to observe lower-energy SEPs just before perihelion 1 , which then increased. Figure 3 shows the events in interval c, including low-energy ions ahead of a coronal mass ejection (CME; Fig. 3b, f, g), the passage of a compression wave after it (Fig. 3c), and a subsequent higher-energy particle event (Fig. 3a).

IS $\odot$ IS observations show an SEP event starting early on 2018-315 and extending to about when the CME arrived at PSP on 2018-316. Particle anisotropies (Fig. 3f) demonstrate that these particles are streaming outward from the Sun. The faster particles arrive first, characteristic of a 'dispersive' SEP event (Fig. 3g) with the differing arrival times giving an estimate of the distance along the magnetic field to the source of their acceleration. For the time-energy slope in Fig. $3 \mathrm{~g}$, we estimate a path length ${ }^{3}$ longer than that of the Parker spiral from PSP at about
$0.25 \mathrm{AU}$, which might be explained by a longer path length associated with magnetic field 'switchbacks' observed by PSP in situ ${ }^{14}$.

Solar observations from the white-light coronagraph on the ' $A$ ' spacecraft of NASA's Solar Terrestrial Relations Observatory (STEREO-A) indicate that the SEP-associated CME started lifting off from the Sun on 2018-314 at about 18:00 UT (Extended Data Fig. 1). Derivation of the CME speed from STEREO-A imaging (Extended Data Fig. 2) reveals that the CME was moving slowly $\left(<400 \mathrm{~km} \mathrm{~s}^{-1}\right)$ from the Sun to PSP, very similar to the surrounding solar wind speed. By propagating this CME flux rope at a constant speed of $380 \mathrm{~km} \mathrm{~s}^{-1}$ from near the Sun to PSP, we find good agreement with the in situ magnetic field observations. Preliminary analysis of this event using shock-modelling techniques ${ }^{15}$ suggests that there was probably no shock on the field lines well connected to PSP. However, a quasi-perpendicular sub-critical shock (Mach number $<3$ ) could have formed over an extended region of the flux rope and perhaps accelerated the protons measured by PSP (A. Kouloumvakos, private communication). This energetic particle event was not seen at any of the 1 AU spacecraft, so such small events may only be observable close to the Sun and therefore much more common than previously thought.

At the end of 2018-318, the solar wind speed increased from about 300 to about $500 \mathrm{~km} \mathrm{~s}^{-1}\left(\right.$ ref. $\left.^{13}\right)$, indicative of a strong dynamic pressure 


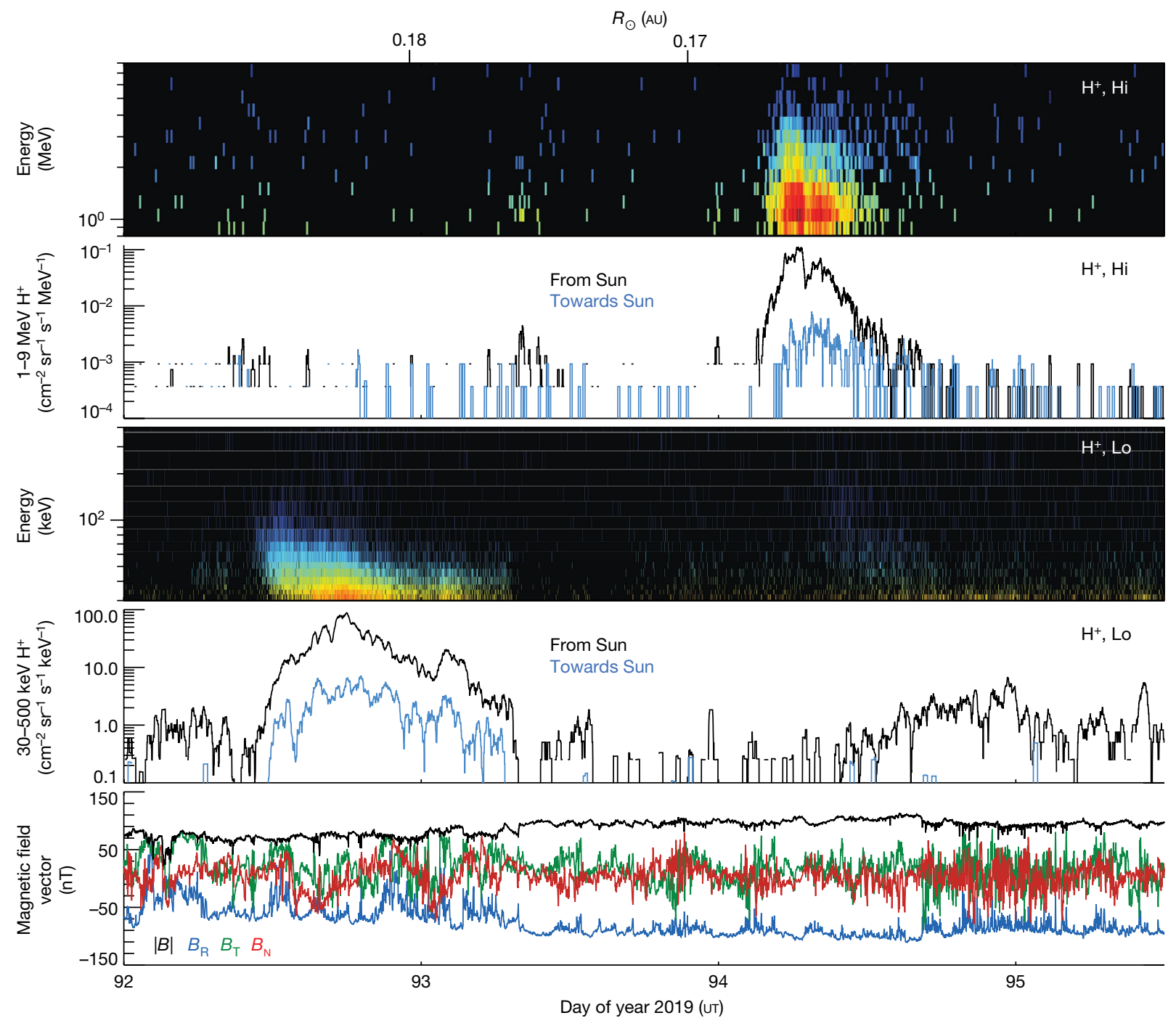

Fig. 4 | Pair of impulsive events near second perihelion. a-e, Two impulsive SEP events (during interval d) near the second perihelion of $\mathrm{PSP}\left(<40 R_{\circ}\right)$ at higher energies $(\mathbf{a}, \mathbf{b})$, lower energies $(\mathbf{c}, \mathbf{d})$, and the magnetic field measured during the events (e). $|B|$ is the magnitude of the magnetic field and $B_{\mathrm{R}}, B_{\mathrm{T}}$ and $B_{\mathrm{N}}$ are the radial, transverse and normal components of the magnetic field, respectively. wave in the solar wind. IS॰IS observed a small enhancement in verylow-energy particles $(<50 \mathrm{keV})$ as this compressional wave passed. This event is the first direct observation of local energization in the IS॰IS observations. Shocks are not required for particle acceleration ${ }^{16}$ and plasma compressions can accelerate particles provided that the particles are able to propagate across, but remain close to, the compression ${ }^{17}$.

The large two-step increase in speed on 2018-318 shows that this compression wave was well on its way to steepening into a forward-reverseshock pair, which probably accelerates the higher-energy (>1 MeV) particles observed from 2018-320 to 2018-324. This is not a CIR as in intervals $a$ and $b$, because it has a much narrower range of foot-point longitudes (see $\mathrm{H}^{+}$count rate at about $300^{\circ}$ in Fig. $2 \mathrm{c}, \mathrm{d}$ ) and does not recur, but instead indicates the interaction of a single fast solar wind stream, possibly associated with or even magnetically initiated by the preceding CME. In any case, as with CIR-associated particle events, the particle isotropy indicates that these ions are trapped on flux tubes, probably with a source more distant than PSP. In fact, at the time the second event was seen, about 1-6 days after the passage of the compression at PSP, the pressure front had expanded outward to heliocentric distances of about 0.6-2 AU, where the shocks probably formed.

Very near perihelion (about 35 solar radii, $R_{\odot}$ ) during PSP orbit 2 (interval d), IS $\odot I S$ observed a unique pair of SEP events (Fig. 4). As PSP is nearly corotational with the Sun near perihelion, the two events are magnetically connected to a common solar source $<5^{\circ}$ apart in longitude. First, on 2019-092 there was a low-energy dispersive event, probably associated with an impulsive source in the low corona. Two days later, on 2019-094, there was a different type of impulsive event, marked by a substantial increase in ions with $>1-\mathrm{MeV}$ energy. Both events exhibit strong, persistent magnetic-field-aligned ions streaming away from the Sun.

The first event, starting on 2019-092, may be associated with disturbances also observed in extreme ultraviolet images from STEREO-A in the vicinity of active region AR2738, as well as multiple type-III radio bursts by both STEREO-A and FIELDS ${ }^{14}$, the magnetic field instrument aboard PSP. This small active region was about $70^{\circ}$ off the nominal magnetic connection of PSP to the Sun. The fluxes of high-energy protons are near background, but we observed a substantial number of heavy high-energy ions and at low energies (about $30 \mathrm{keV}$ per nucleon). $\mathrm{He} / \mathrm{H}$ is about 20 times higher than the event on 2019-094, and increases in $\mathrm{O}$ and $\mathrm{Fe}$ abundances are even greater. These results suggest that this may be a 'Z-rich' event ${ }^{18}$; such events are relatively rare at $1 \mathrm{AU}$.

The second SEP event on 2019-094 also exhibits velocity dispersion and outward streaming, but has many fewer ions $<1 \mathrm{MeV}$ and a 
substantial increase at $>1 \mathrm{MeV}$. As with the event on 2019-092, there is potentially related radio and extreme ultraviolet activity in AR2738. However, the heavy ion abundances were similar to more typical SEP events. The magnetic field observed at PSP (Fig. 4e) between the two events was stronger and considerably smoother than before or after, indicating that this was probably a single, lower $\beta$ (particle pressure/ magnetic pressure) magnetic structure connecting the two events. Further, these observations indicate that processes inside $0.17 \mathrm{AU}$, as suggested by early multi-spacecraft studies in solar cycle 20 , as well as later Helios and STEREO studies ${ }^{19-22}$, enable fast, direct access of SEPs to a wide range of solar longitudes. Later studies that combined in situ data with solar source region observations showed that the smaller, longitudinally distributed SEP events are associated with multiple jet-like coronal emissions ${ }^{23,24}$ close to the source region as well as with more spatially extended eruptions ${ }^{25}$.

IS॰IS observed a rich array of energetic particle phenomena during PSP's first two orbits. Several of these events were not observed by $1 \mathrm{AU}$ spacecraft, and so small events only observable close to the Sun may be much more common than previously thought. With these new data, we are well on the way to resolving the fundamental questions of the origin, acceleration, and transport of SEPs into the heliosphere. Over the next five years, as we head towards solar maximum, PSP will orbit progressively closer to the Sun, ultimately extending our exploration of these critical processes to less than $10 R_{\odot}$.

\section{Online content}

Any methods, additional references, Nature Research reporting summaries, source data, extended data, supplementary information, acknowledgements, peer review information; details of author contributions and competing interests; and statements of data and code availability are available at https://doi.org/10.1038/s41586-019-1811-1.

1. Fox, N. J. et al. The Solar Probe Plus mission: humanity's first visit to our star. Space Sci. Rev. 204, 7-48 (2016).

2. Mason, G. M. ${ }^{3} \mathrm{He}$-rich solar energetic particle events. Space Sci. Rev. 130, 231-242 (2007)

3. Desai, M. I. \& Giacalone, J. Large gradual solar energetic particle events. Living Rev. Sol. Phys. 13, 3 (2016)

4. Reames, D. V. Solar Energetic Particles: A Modern Primer on Understanding Sources, Acceleration and Propagation (Springer, 2017).
5. Cane, H. V., Richardson, I. G. \& von Rosenvinge, T. T. A study of solar energetic particle events of 1997-2006: their composition and associations. J. Geophys. Res. Space Phys. 115, A08101 (2010)

6. Wibberenz, G. \& Cane, H. V. Multi-spacecraft observations of solar flare particles in the inner heliosphere. Astrophys. J. 650, 1199-1207 (2006).

7. McComas, D. J. et al. Integrated Science Investigation of the Sun (ISIS): design of the energetic particle investigation. Space Sci. Rev. 204, 187-256 (2016).

8. Kasper, J. C. et al. Solar Wind Electrons Alphas and Protons (SWEAP) investigation: design of the solar wind and coronal plasma instrument suite for Solar Probe Plus. Space Sci. Rev. 204, 131-186 (2016).

9. Bale, S. D. et al. The FIELDS instrument suite for Solar Probe Plus. Measuring the coronal plasma and magnetic field, plasma waves and turbulence, and radio signatures of solar transients. Space Sci. Rev. 204, 49-82 (2016).

10. Vourlidas, A. et al. The Wide-field Imager for Solar Probe Plus (WISPR). Space Sci. Rev. 204, 83-130 (2016).

11. Pizzo, V. A three-dimensional model of corotating streams in the solar wind, 1. Theoretical foundations. J. Geophys. Res. 83, 5563 (1978).

12. Gosling, J. Corotating and transient solar wind flows in three dimensions. Annu. Rev. Astron. Astrophys. 34, 35-73 (1996).

13. Kasper, J. C. et al. Alfvénic velocity spikes and rotational flows in the near-Sun solar wind. Nature https://doi.org/10.1038/s41586-019-1813-z (2019).

14. Bale, S. D. et al. Highly structured slow solar wind emerging from an equatorial coronal hole. Nature https://doi.org/10.1038/s41586-019-1818-7 (2019).

15. Kouloumvakos, A. et al. Connecting the properties of coronal shock waves with those of solar energetic particles. Astrophys. J. 876, 80 (2019).

16. Chotoo, K. et al. The suprathermal seed population for corotating interaction region ions at 1 au deduced from composition and spectra of $\mathrm{H}^{+}, \mathrm{He}^{++}$, and $\mathrm{He}^{+}$observed on Wind. J. Geophys. Res. Space Phys. 105, 23107-23122 (2000).

17. Giacalone, J., Jokipii, J. R. \& Kota, J. Particle acceleration in solar wind compression regions. Astrophys. J. 573, 845-850 (2002).

18. Zwickl, R. D., Roelof, E. C., Gold, R. E., Krimigis, S. M. \& Armstrong, T. P. Z-rich solar particle event characteristics 1972-1976. Astrophys. J. 225, 281-303 (1978).

19. Reinhard, R. \& Wibberenz, G. Propagation of flare protons in the solar atmosphere. Sol. Phys. 36, 473-494 (1974).

20. Kallenrode, M. B. Particle propagation in the inner heliosphere. J. Geophys. Res. Space Phys. 98, 19037-19047 (1993).

21. Richardson, I. G., von Rosenvinge, T. T. \& Cane, H. V. The properties of solar energetic particle event-associated coronal mass ejections reported in different CME catalogs. Sol. Phys. 290, 1741-1759 (2015).

22. Wiedenbeck, M. E. et al. Observations of solar energetic particles from ${ }^{3} \mathrm{He}$-rich events over a wide range of heliographic longitude. Astrophys. J. 762, 54 (2013).

23. Bučik, R. et al. ${ }^{3} \mathrm{He}$-rich solar energetic particles from sunspot jets. Astrophys. J. Lett. 869 21 (2018)

24. Bučik, R., et al. Multi-spacecraft observations of recurrent ${ }^{3} \mathrm{He}$-rich solar energetic particles. Astrophys. J. 786, 71 (2014).

25. Nitta, N. V., Mason, G. M., Wang, L., Cohen, C. M. S. \& Wiedenbeck, M. E. Solar sources of ${ }^{3} \mathrm{He}$-rich solar energetic particle events in solar cycle 24. Astrophys. J. 806, 235 (2015).

Publisher's note Springer Nature remains neutral with regard to jurisdictional claims in published maps and institutional affiliations.

(c) The Author(s), under exclusive licence to Springer Nature Limited 2019 


\section{Article}

\section{Data availability}

All data used in this study is available to the public via NASA's Space Physics Data Facility (SPDF) at https://spdf.gsfc.nasa.gov/.

26. Howard, R. A. Sun Earth Connection Coronal and Heliospheric Investigation (SECCHI). Adv. Space Res. 29, 2017-2026 (2002).

27. Thernisien, A., Vourlidas, A. \& Howard, R. Forward modeling of coronal mass ejections using STEREO/SECCHI data. Sol. Phys. 256, 111 (2009).

28. Rouillard, A. P. et al. A solar storm observed from the Sun to Venus using the STEREO, Venus Express, and MESSENGER spacecraft. J. Geophys. Res. 114, A07106 (2010)

29. Wood, B. \& Howard, R. An empirical reconstruction of the 2008 April 26 coronal mass ejection. Astrophys. J. 702, 901-910 (2009).

30. Isavnin, A. FRiED: a novel three-dimensional model of coronal mass ejections. Astrophys. J. 833, 10 (2016)

Acknowledgements We are indebted to everyone who helped make the PSP mission possible. In particular, we thank all of the scientists, engineers, technicians, and administrative support people across all of the IS॰IS institutions that produced and supported the IS॰IS instrument suite and support its operations and the scientific analysis of its data. This work was supported as a part of the PSP mission under contract NNN06AA01C. S.D.B. acknowledges the support of the Leverhulme Trust Visiting Professorship programme and A.P.R. acknowledges financial support from the ANR project COROSHOCK ANR-17-CE31-0006-01 and from the ERC project SLOW_SOURCE - DLV-819189.

Author contributions D.J.M. is IS॰IS Principal Investigator (PI) and led the data analysis and writing of the study. E.R.C. is IS॰IS Deputy PI, helped develop EPI-Hi, and participated in the data analysis. C.M.S.C. helped develop EPI-Hi and participated in the data analysis. A.C.C. helped develop EPI-Hi and participated in the data analysis. A.J.D. helped develop EPI-Hi and participated in the data analysis. M.I.D. participated in the data analysis. J.G. participated in the data analysis. M.E.H. helped develop EPI-Lo and participated in the data analysis. C.J.J. produced Figs. 3, 4 and participated in the data analysis. S.M.K. participated in the data analysis. A.W.L. helped develop EPI-Hi and participated in the data analysis. R.A.L. helped develop EPI-Hi and participated in the data analysis. O.M. participated in the data analysis. W.H.M. participated in the data analysis. R.L.M. led the development of EPI-Lo and participated in the data analysis. R.A.M. helped develop EPI-Hi and participated in the data analysis. D.G.M. helped develop EPI-Lo and participated in the data analysis. A.P. participated in the data analysis. J.S.R. helped develop EPI-Hi and participated in the data analysis. E.C.R. participated in the data analysis. N.A.S. led the development of the IS॰IS Science Operations Center and participated in the data analysis. E.C.S. helped develop EPI-Hi and participated in the data analysis. J.R.S. led the development of the analysis tool, produced Figs. 1, 2, and participated in the data analysis. M.E.W. led the development of EPI-Hi and participated in the data analysis. S.D.B. is FIELDS PI and participated in the data analysis. J.C.K. is SWEAP PI and participated in the data analysis. A.W.C. helped develop SWEAP and participated in the data analysis. K.E.K. helped develop SWEAP and participated in the data analysis. R.J.M. helped develop FIELDS and participated in the data analysis. M.P. helped develop FIELDS and participated in the data analysis. M.L.S. helped develop SWEAP and participated in the data analysis. A.P.R. led the CME simulation work and participated in the data analysis.

Competing interests The authors declare no competing interests.

Additional information

Correspondence and requests for materials should be addressed to D.J.M.

Peer review information Nature thanks Hazel Bain and Monica Laurenza for their contribution

to the peer review of this work.

Reprints and permissions information is available at http://www.nature.com/reprints. 


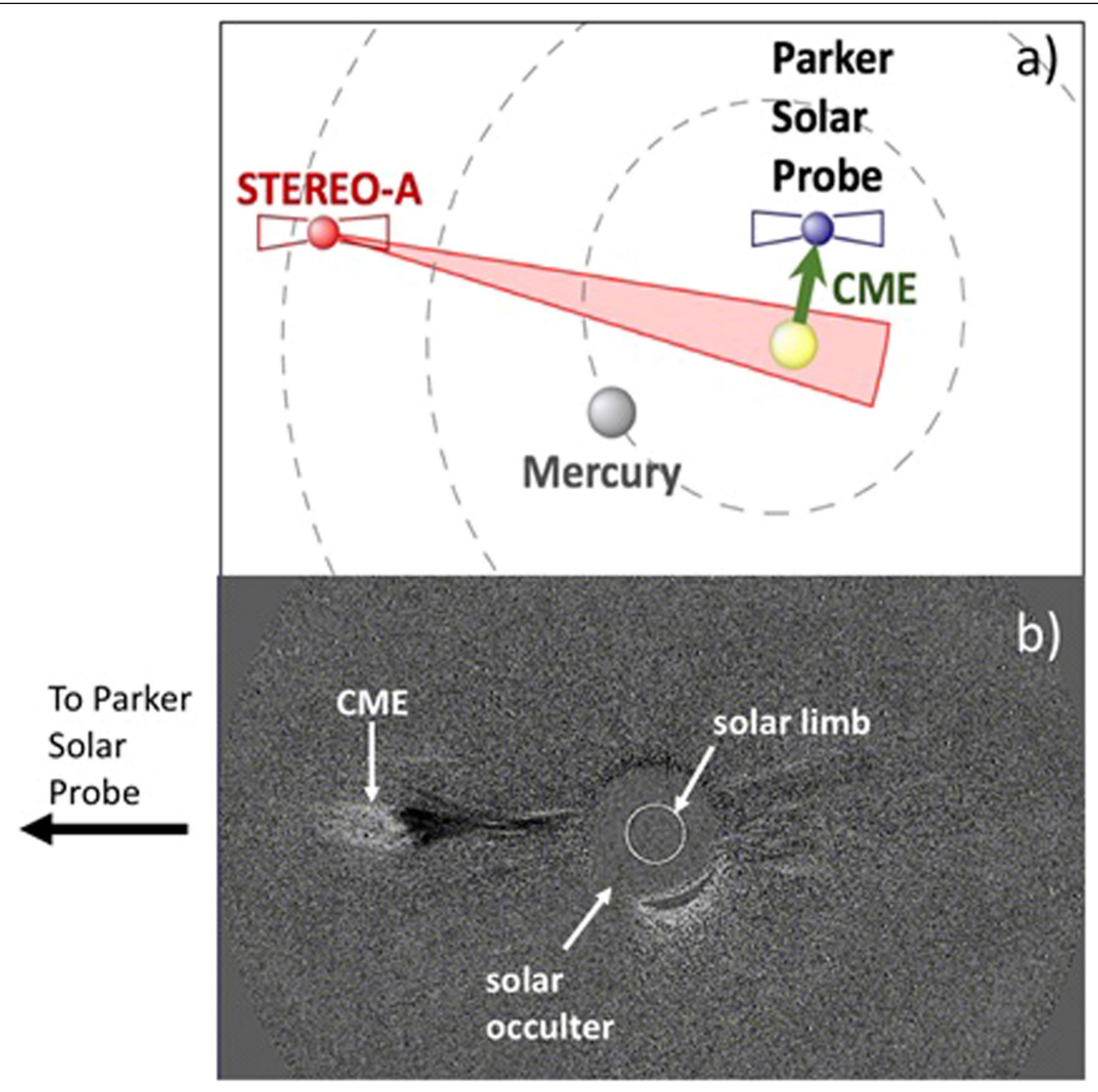

Extended Data Fig. 1 | Viewing geometry and observation of coronal mass ejection. a, A view of the ecliptic plane from solar north at 14:00 10 November 2018 UT showing the relative positions of STEREO-A and PSP. The dashed curves, from innermost to outermost, represent the orbits of Mercury, Venus and Earth. The red area shows the field of view of the COR-2 instrument onboard STEREO-A. A CME off the east limb of the Sun as viewed from STEREO-A would be roughly propagating towards PSP. This CME very gradually entered the field of view of COR-2, part of the SECCHI suite of imaging instruments ${ }^{26}$ aboard the STEREO spacecraft. $\mathbf{b}$, A running-difference image of the CME taken at 02:39 UT on 11 November 2018 by COR-2A (a visible-light coronagraph), extending in the plane of the sky from $2 R_{\odot}$ to $15 R_{\circ}$, provided images during the entire acceleration phase of the CME. This CME entered COR-2A around 18:00 UT on 10 November 2018 and transited through the COR-2 field of view over about $12 \mathrm{~h}$. 


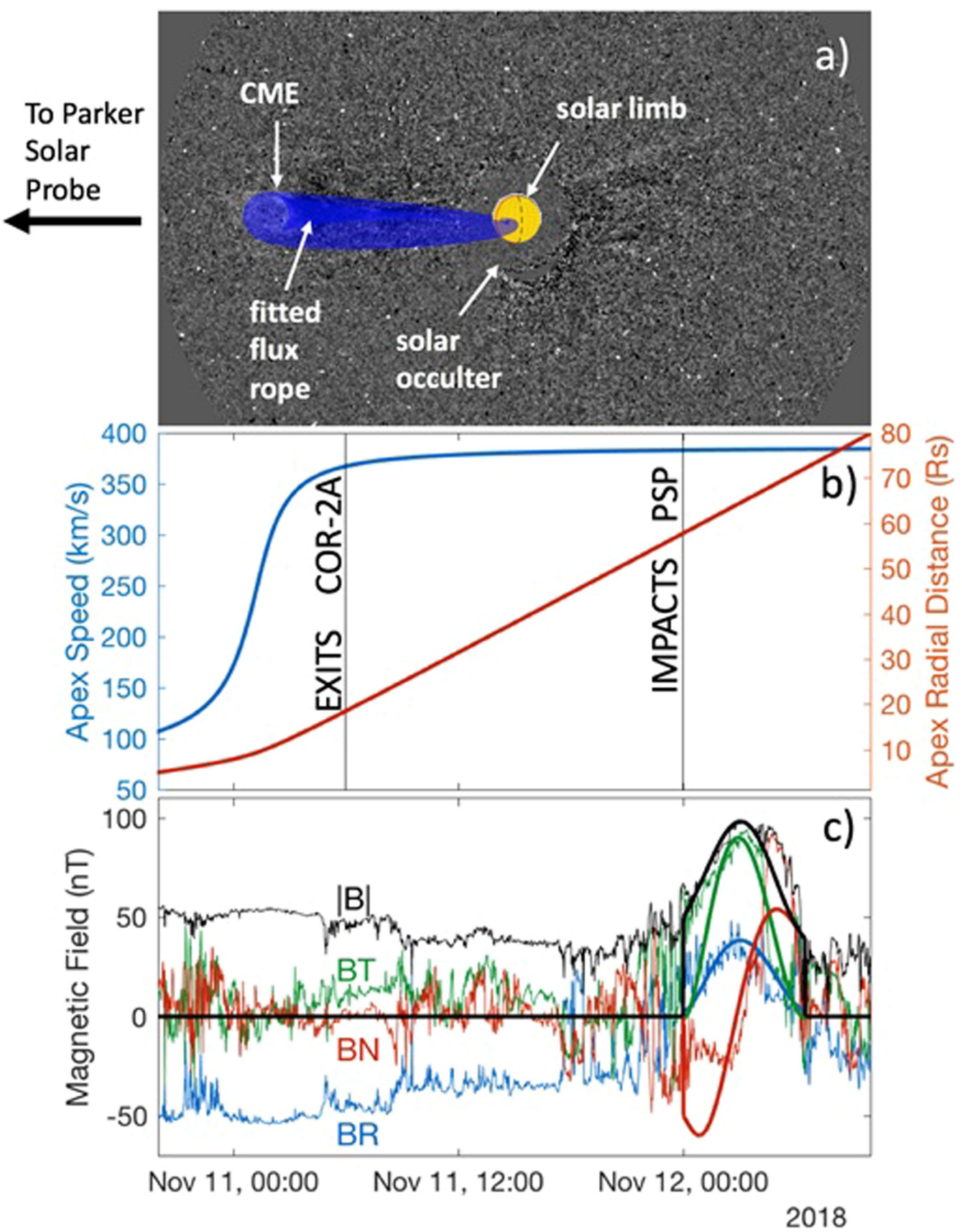

Extended Data Fig. 2 | CME model and comparison to magnetic field data. a, The same as in Extended Data Fig. $1 \mathrm{~b}$ but with superposed fitted flux-rope shape of the CME at 02:3911 November 2018 UT when the CME had passed halfway through the COR-2A field of view. The CME is very weak and no shocksheath structure can be identified in these images. The typical aspect of the CME in the image results from the line-of-sight integration of plasma distribution on a bent toroid such that its major axis is located in a plane containing the observing spacecraft (see very similar events in refs. ${ }^{27,28}$ ). b, The position (red) and speed (blue) of the apex of the flux-rope model was derived by iteratively comparing each synthetic image produced by the threedimensional model with each available COR-2A image. A functional form (arctangent) was imposed for the flux rope's varying speed. The fitted CME structure assumed here is a bent toroid with an exponential increase of its cross-sectional area from foot point to apex as in ref. ${ }^{29}$. The speed was derived by fitting a hyperbolic tangent to the modelled CME position. The speed increases rapidly from under $100 \mathrm{~km} \mathrm{~s}^{-1}$ at 18:00 10 November $2018 \mathrm{UT}$ to over $350 \mathrm{~km} \mathrm{~s}^{-1}$ when it exited the COR-2A field of view at around 6:00 uT on 11 November.c, An internal magnetic field structure was expressed analytically inside the envelope of the fitted CME (smooth curves) as in ref. ${ }^{30}$, but here keeping a simple circular cross-section of the flux rope. By propagating this flux rope at a constant speed of $380 \mathrm{~km} \mathrm{~s}^{-1}$ from the time it exits the COR-2 field of view, we predict the CME reaches PSP on 12 November 2018. The predicted arrival time and the magnetic properties of the CME (thick smooth line) are in good agreement with those measured in situ by the FIELDS (magnetic field data; thin lines) and SWEAP instruments (not shown). We therefore conclude that the fitting procedure presented here provides a good description of the evolution of the CME from the upper corona to PSP. 


\section{Alfvénic velocity spikes and rotational flows in the near-Sun solar wind}

https://doi.org/10.1038/s41586-019-1813-z

Received: 18 July 2019

Accepted: 17 October 2019

Published online: 4 December 2019

\author{
J. C. Kasper ${ }^{1,2 *}$, S. D. Bale ${ }^{3,4,5}$, J. W. Belcher ${ }^{6}$, M. Berthomier ${ }^{7}$, A. W. Case ${ }^{2}$, B. D. G. Chandran ${ }^{8,9}$, \\ D. W. Curtis ${ }^{4}$, D. Gallagher ${ }^{10}$, S. P. Gary ${ }^{11}$, L. Golub ${ }^{2}$, J. S. Halekas ${ }^{12}$, G. C. Ho ${ }^{13}$, T. S. Horbury ${ }^{5}$, \\ Q. Hu ${ }^{14}$, J. Huang', K. G. Klein ${ }^{15,16}$, K. E. Korreck² ${ }^{2}$ D. E. Larson ${ }^{4}$, R. Livi ${ }^{4}$, B. Maruca ${ }^{17,18}$, \\ B. Lavraud ${ }^{19}$, P. Louarn ${ }^{19}$, M. Maksimovic ${ }^{20}$, M. Martinovic ${ }^{15}$, D. McGinnis ${ }^{12}$, N. V. Pogorelov ${ }^{14}$, \\ J. D. Richardson ${ }^{6}$, R. M. Skoug ${ }^{11}$, J. T. Steinberg" ${ }^{11}$, M. L. Stevens' ${ }^{2}$, A. Szabo ${ }^{21}$, M. Velli22, \\ P. L. Whittlesey ${ }^{4}$, K. H. Wright ${ }^{23}$, G. P. Zank ${ }^{14}$, R. J. MacDowall ${ }^{21}$, D. J. McComas ${ }^{24}$, \\ R. L. McNutt Jr ${ }^{13}$, M. Pulupa ${ }^{4}$, N. E. Raouafi ${ }^{13}$ \& N. A. Schwadron ${ }^{8,9}$
}

\begin{abstract}
The prediction of a supersonic solar wind ${ }^{1}$ was first confirmed by spacecraft near Earth $^{2,3}$ and later by spacecraft at heliocentric distances as small as 62 solar radii ${ }^{4}$. These missions showed that plasma accelerates as it emerges from the corona, aided by unidentified processes that transport energy outwards from the Sun before depositing it in the wind. Alfvénic fluctuations are a promising candidate for such a process because they are seen in the corona and solar wind and contain considerable energy $^{5-7}$. Magnetic tension forces the corona to co-rotate with the Sun, but any residual rotation far from the Sun reported until now has been much smaller than the amplitude of waves and deflections from interacting wind streams ${ }^{8}$. Here we report observations of solar-wind plasma at heliocentric distances of about 35 solar radii ${ }^{9-11}$, well within the distance at which stream interactions become important. We find that Alfvén waves organize into structured velocity spikes with duration of up to minutes, which are associated with propagating S-like bends in the magnetic-field lines. We detect an increasing rotational component to the flow velocity of the solar wind around the Sun, peaking at 35 to 50 kilometres per second-considerably above the amplitude of the waves. These flows exceed classical velocity predictions of a few kilometres per second, challenging models of circulation in the corona and calling into question our understanding of how stars lose angular momentum and spin down as they age $\mathrm{e}^{12-14}$.
\end{abstract}

The Parker Solar Probe (PSP) launched in August 2018 on a Delta IV Heavy rocket. The high energy of the launch, combined with a gravitational assist from Venus in September 2018, placed PSP into an eccentric orbit with a period of 147 days and a perihelion at a heliocentric distance of $r=35.7 R_{\odot}\left(R_{\odot}\right.$, solar radius) nearly a factor of two closer to the Sun than any previous mission ${ }^{4}$. This study uses observations made by instruments on the spacecraft during the first two encounters with the Sun, in November 2018 and April 2019. Whereas the instruments collect data at a low rate far from the Sun, the primary science collection at a high rate occurs during the encounter phase of each orbit at $r<54 R_{\odot}$ $(0.25 \mathrm{AU})$. Encounter one (E1) lasted from 31 October to 12 November
2018, with the first perihelion occurring at 03:27 UT on 6 November. During these two encounters the longitude of PSP relative to the rotating surface of the Sun barely changed; PSP essentially dove down into, and then rose straight up from, a single narrow region above the Sun. E1 and E2 data thus describe a handful of specific solar-wind streams. Nearly two million thermal-energy distribution functions of the solarwind protons were recorded during E1, and more than three times that number during E2 (Fig. 1, Extended Data Fig. 1). From these distribution functions, the bulk properties of solar-wind protons-such as the velocity, density and temperature-are derived. Within any hour interval, the distribution of the radial solar-wind speed, $V_{\mathrm{pR}}$, was strongly peaked at a

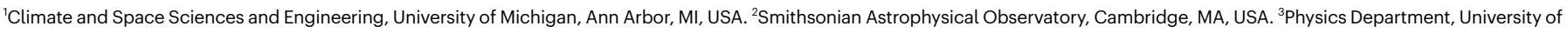
California, Berkeley, CA, USA. ${ }^{4}$ Space Sciences Laboratory, University of California, Berkeley, CA, USA. ${ }^{5}$ The Blackett Laboratory, Imperial College London, London, UK. ${ }^{6}$ Kavli Center for Astrophysics and Space Sciences, Massachusetts Institute of Technology, Cambridge, MA, USA. ${ }^{7}$ Laboratoire de Physique des Plasmas, CNRS, Sorbonne Université, Ecole Polytechnique,

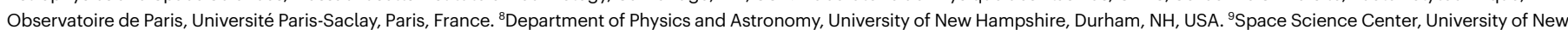
Hampshire, Durham, NH, USA. ${ }^{10}$ Heliophysics and Planetary Science Branch ST13, Marshall Space Flight Center, Huntsville, AL, USA. "Los Alamos National Laboratory, Los Alamos, NM, USA. ${ }^{12}$ Department of Physics and Astronomy, University of lowa, IA, USA. ${ }^{13}$ Johns Hopkins University Applied Physics Laboratory, Laurel, MD, USA. ${ }^{14}$ Department of Space Science and Center for Space Plasma and Aeronomic Research, University of Alabama in Huntsville, Huntsville, AL, USA. ${ }^{15}$ Lunar and Planetary Laboratory, University of Arizona, Tucson, AZ, USA. ${ }^{16}$ Department of

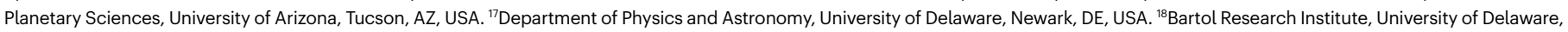

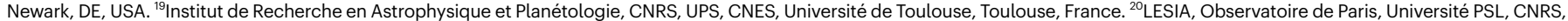
Sorbonne Université, Université de Paris, Meudon, France. ${ }^{21} \mathrm{NASA} /$ Goddard Space Flight Center, Greenbelt, MD, USA. ${ }^{22}$ Department of Earth, Planetary and Space Sciences, University of California, Los Angeles, CA, USA. ${ }^{23}$ Universities Space Research Association, Science and Technology Institute, Huntsville, AL, USA. ${ }^{24}$ Department of Astrophysical Sciences, Princeton University, Princeton, NJ, USA. *e-mail: jckasper@umich.edu 


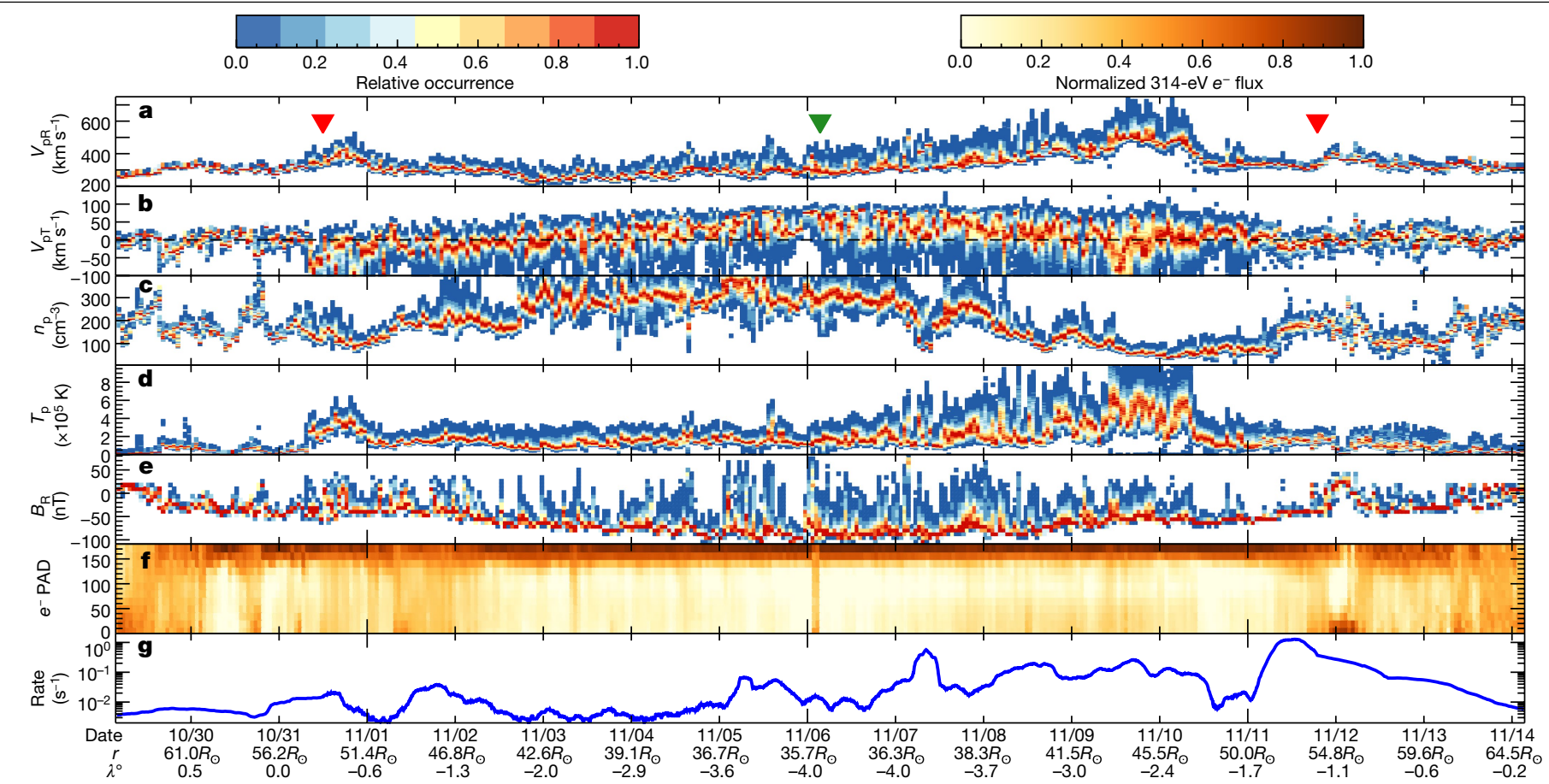

Fig. 1 Overview of the first encounter of PSP with the Sun. a, Relative occurrence rate of the proton radial speed $V_{\mathrm{PR}}$ in one-hour intervals. Red triangles show the start and end of the high-rate data collection below $54 R_{\odot}$ and the green triangle indicates a perihelion at $35.7 R_{\odot} . \mathbf{b}-\mathbf{f}$, The same for $V_{\mathrm{pT}}$ in the solar equatorial plane $(\mathbf{b})$, the proton number density $n_{\mathrm{p}}(\mathbf{c})$, the proton

temperature $T_{\mathrm{p}}(\mathbf{d})$, the radial component of magnetic field $B_{\mathrm{R}}(\mathrm{e})$, the electron pitch-angle distribution (PAD) (f) and the $20-200 \mathrm{keV}$ proton rate $(\mathrm{g})$. The date (month/day), distance $r$ and latitude $\lambda$ relative to the solar equator are indicated at daily intervals.

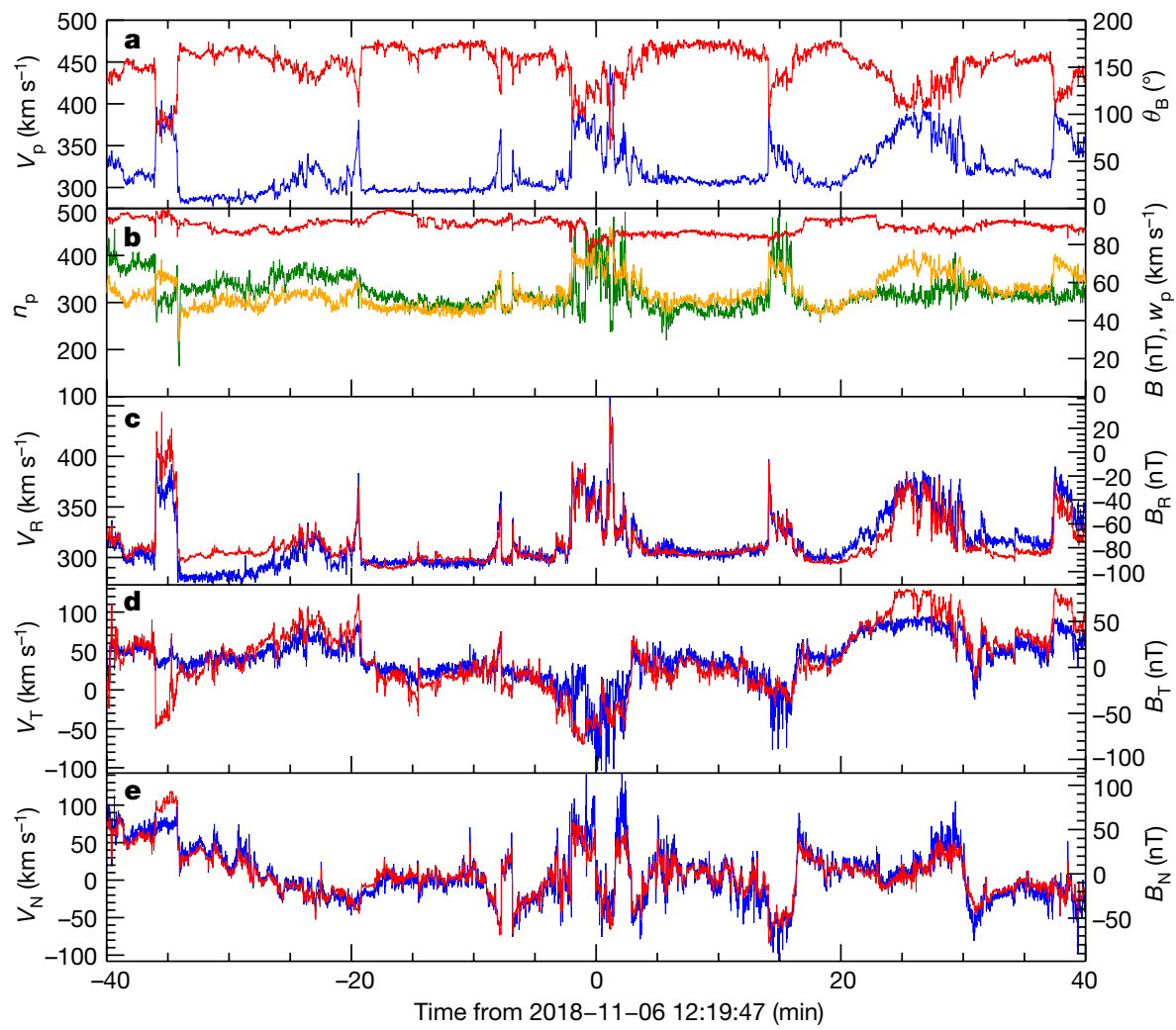

Fig. 2 | Solar-wind fluctuations near the closest approach. Near-Sun fluctuations meet the Alfvénic criteria, but are organized into structures and contain density enhancements. a, Magnitude of $V_{\mathrm{pR}}$ (blue) and angle $\theta_{\mathrm{B}}$ of $\mathbf{B}$ from the radial direction outwards. $\mathbf{b}$, Magnitudes of $n_{\mathrm{p}}$ (green), $B$ (red) and the proton thermal speed $w_{\mathrm{p}}$ (yellow).c-e, Variation of each vector component of the velocity (blue) and magnetic field (red) in the radial direction ( $R$ ), the transverse direction in the solar equatorial plane $(\mathrm{T})$ and the normal to $\mathrm{R}$ and $\mathrm{T}(\mathrm{N})$. Since the orbit of PSP is within a few degrees of the solar equator, $\mathrm{N}$ points approximately north, perpendicular to the equatorial plane. There is a baseline solar-wind speed of about $300 \mathrm{~km} \mathrm{~s}^{-1}$ and jets where $V_{\mathrm{p}}$ jumps by about $100 \mathrm{~km} \mathrm{~s}^{-1}$. The fluctuations are highly Alfvernic, with equal energy in the field and the flow, but they are organized into structures instead of being randomly distributed, and there is evidence of compressions. 


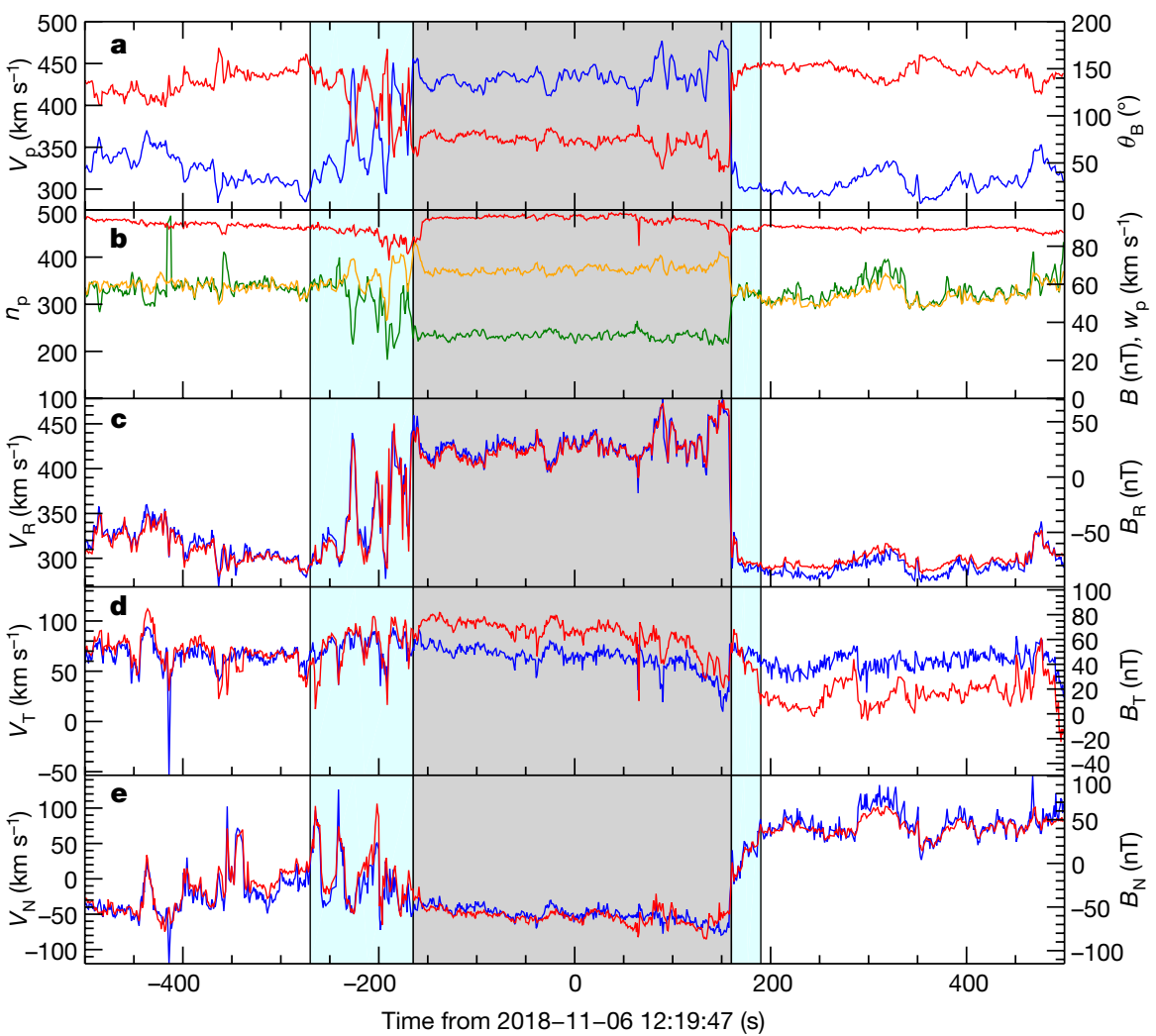

Fig. 3 | A closer look at a velocity spike. The formatting is as in Fig. 2, but focused on a single 1,000-s interval. The left blue region indicates the 105 -s period in which PSP moved from the ambient plasma into the spike. The central core of the spike is indicated by the grey region and lasted for $325 \mathrm{~s}$; it is characterized by a steady but disturbed flow and a field with a large rotation in B to $\theta_{\mathrm{B}} \approx 70^{\circ}$ and a jump in flow to $343 \mathrm{~km} \mathrm{~s}^{-1}$. The return from the core spike into ambient solar wind is marked by the second blue region and lasted $30 \mathrm{~s}$. minimum value, with a one-sided tail extending to larger $V_{\mathrm{pR}}$ values. $V_{\mathrm{pR}}$ reached its minimum of $200 \mathrm{~km} \mathrm{~s}^{-1}$ about a quarter of the way though $\mathrm{E} 1$ and then rose steadily to about $600 \mathrm{~km} \mathrm{~s}^{-1}$. Numerical simulations and simple extrapolations of the observed photospheric magnetic field suggest that PSP spent all of E1 south of the global heliospheric current sheet, in a region with inward magnetic polarity $\left(B_{R}<0\right)^{15}$. Near the start and end of E1, PSP sampled slow wind from near the global heliospheric current sheet. Closer to the Sun, PSP first observed very slow wind and then fast wind, both of which are thought to emerge from a low-latitude coronal hole ${ }^{15}$. Below $40 R_{\odot}, V_{\mathrm{pT}}$ (the transverse component of the proton velocity in the equatorial plane of the Sun) has a net positive value, which peaks at the closest approach. This flow may be the long-sought signature of plasma co-rotation in the corona. The density peaks in the slowest wind, at a value of approximately $400 \mathrm{~cm}^{-3}$, about 50 times higher than values typically observed at $1 \mathrm{AU}$, as expected from mass conservation and spherical expansion. The proton temperature, $T_{\mathrm{p}}$, and $V_{\mathrm{pR}}$ remain positively correlated ${ }^{16}$. At perihelion the protons are about 4 times hotter than protons with similar $V_{\mathrm{pR}}$ at $1 \mathrm{AU}$, consistent with radial scalings reported from earlier missions ${ }^{4}$. The radial component of the magnetic field, $B_{R}$, increases in magnitude with proximity to the Sun but unexpectedly changes sign many times. The pitch-angle $(\theta)$ distribution for electrons (that is, the number of electrons at a given energy as a function of their angle relative to $\mathbf{B}$ ) is a valuable diagnostic of these changes in the direction of $\mathbf{B}$. Here we show the pitch-angle distribution in a 22-eV-wide energy channel centred on $314 \mathrm{eV}$, well above the electron thermal energy. The sharp peak near $180^{\circ}$ corresponds to the strahl, a beam of super-thermal electrons that travel away from the Sun along magnetic-field lines. Near the Sun, the strahl evolves towards small $\sin \theta$ values because of magnetic-moment conservation ${ }^{17}$. If the reversals in $B_{\mathrm{R}}$ seen by PSP result from the spacecraft's crossing between open field lines (connected to the Sun at only one end) with different signs of $B_{\mathrm{R}}$ back at the Sun, then the strahl would flip between $180^{\circ}$ and $0^{\circ}$ each time $B_{\mathrm{R}}$ changed sign. Instead, every time $B_{\mathrm{R}}$ flips, the strahl maintains its $180^{\circ}$ orientation, clearly indicating that the reversals in $B_{\mathrm{R}}$ are due to S-like bends in the magnetic-field lines (Extended Data Fig. 2). Closed field lines with both ends connected to the Sun and strahl travelling in both parallel and antiparallel directions to $\mathbf{B}$ are seen during the arrival of a coronal mass ejection on 11 November at 23:50 UT, following an enhancement in the number of energetic particles ${ }^{18}$.

Figure 2 shows a timeseries of 80 min of observations several hours after perihelion, illustrating typical velocity and magnetic-field fluctuations. About half the time, B points radially inwards towards the Sun and the velocity $V$ remains at a relatively constant $300 \mathrm{~km} \mathrm{~s}^{-1}$. The remaining time includes seven distinct intervals in which $\mathbf{B}$ rotates away from its radial-inward orientation, $V_{\mathrm{PR}}$ simultaneously jumps and $\mathbf{V}$ also rotates, linking the one-sided tail in $V_{\mathrm{pR}}$ with the reversals in polarity seen in the E1 overview. These jumps in flow associated with rotations in $\mathbf{B}$ and $\mathbf{V}$ are similar to one-sided Alfvénic structures that were first seen farther from the Sun ${ }^{6,7}$. The spikes seen by PSP are different in that they have larger amplitudes and are often associated with an increase in the proton density, $n_{\mathrm{p}}$, indicating that the spikes have a non-Alfvénic component. The correlated variations in the components of $B$ and $V$, their relative amplitudes and the constant value of $|\mathbf{B}|$ are consistent with large-amplitude, spherically polarized Alfvén waves propagating through the plasma in the anti-Sunward direction, similar to earlier observations ${ }^{5,19}$. We can classify this wind stream (and indeed much of E1) as Alfvénic slow solar wind ${ }^{20}$.

About 1,000 long-duration ( $>10 \mathrm{~s}$ ) and isolated velocity spikes with large rotations in $\mathbf{B}$ were identified in $\mathrm{E} 1$ (about half as many were seen in E2.) Often the spikes can be separated chronologically into a core region with plasma conditions that are very different from those of the ambient solar wind but relatively constant, a comparatively short 


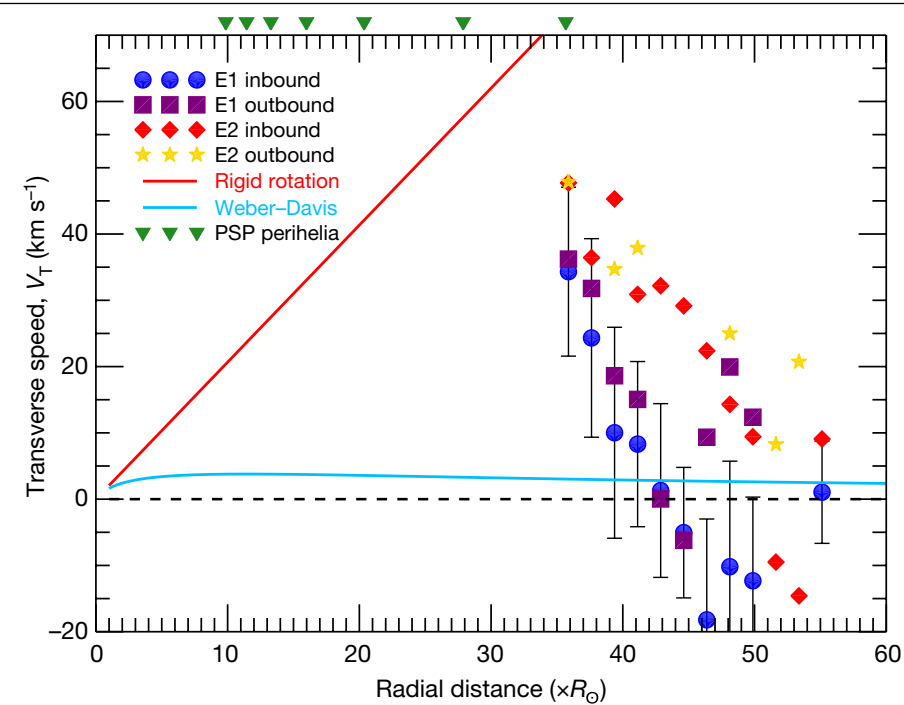

Fig. 4 | Large circulation of solar wind observed near the Sun. Averaged rotational (or azimuthal) flow, $V_{\mathrm{p}}$, over $1.75 R_{\odot}$ intervals during $\mathrm{E} 1$ (inbound in blue, with error bars indicating the standard deviation from all observations; outbound in purple) and E2 (inbound in red; outbound in yellow) as a function of radial distance. Each symbol represents the average over at least 10,000 observations, and the values closest to perihelion are averaged over $60,000-$ 230,000 observations. Error bars for E1 inbound show one standard deviation of the individual observations and are representative of the variation for the other three phases. The uncertainty in the mean of $V_{\mathrm{pT}}$ is much smaller than the marker size. Current and upcoming perihelia are shown with green triangles. Lines indicate no rotation (dashed), rigid co-rotation everywhere (red) and the axisymmetric Weber-Davis model (blue).

transition region on one side of the core, and a longer transition region on the other side containing large-amplitude fluctuations (see Fig. 3). During the 105-s transition at the beginning of this spike, the flow underwent seven large oscillations with an amplitude of $150 \mathrm{~km} \mathrm{~s}^{-1}$, which possibly resulted from a Kelvin-Helmholtz instability.

Equally unexpected as the spikes and $B_{\mathrm{R}}$ reversals are the large-amplitude and sustained positive rotational velocities seen below $40 R_{\odot}$ for $\mathrm{E} 1$ and $50 R_{\odot}$ for $\mathrm{E} 2$ (Fig. 4). Net rotation has been reported farther from the Sun, but it was of the same order as instrument error and much smaller than the standard deviation in flow due to fluctuations and stream interactions ${ }^{8,21}$. Here $V_{\mathrm{pT}}$ rises to $35 \mathrm{~km} \mathrm{~s}^{-1}$ (E1) and $50 \mathrm{~km} \mathrm{~s}^{-1}$ (E2). This is much greater than the variance from fluctuations including the velocity spikes, there is no evidence of stream interactions, and these values are much greater than the precision in the averaged flows (less than $0.5 \mathrm{~km} \mathrm{~s}^{-1}$ ) and the absolute error in the flow due to a pointing error (less than $3 \mathrm{~km} \mathrm{~s}^{-1}$ ) (see Methods). These are the first in situ observations of net rotational flow in the solar wind that are significantly above fluctuations and uncertainty.

Some level of rotational flow has always been expected in the solar wind near the Sun, as magnetic tension in the corona should force the plasma to rotate as the Sun spins. However, the large rotational velocities measured here greatly exceed the value calculated by the axisymmetric Weber-Davis model ${ }^{13}$, posing a major challenge to our understanding of the dynamics of the near-Sun solar wind. Determining the origin of these tangential flows will be essential for understanding how the Sun loses angular momentum and spins down as it ages ${ }^{12,14,22}$. Further studies of the angular momentum should include magnetic fields, waves and different ions. Future PSP orbits will clarify the extent to which these large rotational flows characterize other solar-wind streams. These orbits will also provide critical additional diagnostics of the state of the plasma, including turbulence, velocity spikes, temperature anisotropy and particle velocity distribution functions, at heliocentric distances as small as $9.86 R_{\odot}$.

\section{Online content}

Any methods, additional references, Nature Research reporting summaries, source data, extended data, supplementary information, acknowledgements, peer review information; details of author contributions and competing interests; and statements of data and code availability are available at https://doi.org/10.1038/s41586-019-1813-z.

1. Parker, E. N. Dynamics of the interplanetary gas and magnetic fields. Astrophys. J. 128, 664-676 (1958)

2. Gringauz, K. I., Bezrokikh, V. V., Ozerov, V. D. \& Rybchinskii, R. E. A study of the interplanetary ionized gas, high-energy electrons and corpuscular radiation from the Sun by means of the three-electrode trap for charged particles on the second Soviet cosmic rocket. Sov. Phys. Dokl. 5, 361-364 (1960).

3. Bonetti, A., Bridge, H. S., Lazarus, A. J., Rossi, B. \& Scherb, F. Explorer 10 plasma measurements. J. Geophys. Res. 68, 4017-4063 (1963).

4. Marsch, E. et al. Solar wind protons - three-dimensional velocity distributions and derived plasma parameters measured between 0.3 and 1 AU. J. Geophys. Res. 87, 52-72 (1982).

5. Belcher, J. W. \& Davis, L. Jr Large-amplitude Alfvén waves in the interplanetary medium, 2. J. Geophys. Res. 76, 3534-3563 (1971).

6. Gosling, J. T., McComas, D. J., Roberts, D. A. \& Skoug, R. M. A one-sided aspect of Alfvenic fluctuations in the solar wind. Astrophys. J. Lett. 695, 213-216 (2009).

7. Horbury, T. S., Matteini, L. \& Stansby, D. Short, large-amplitude speed enhancements in the near-Sunfast solar wind. Mon. Not. R. Astron. Soc. 478, 1980-1986 (2018).

8. Pizzo, V. et al. Determination of the solar wind angular momentum flux from the HELIOS data - an observational test of the Weber and Davis theory. Astrophys. J. 271, 335-354 (1983).

9. Fox, N. J. et al. The solar probe plus mission: humanity's first visit to our star. Space Sci. Rev. 204, 7-48 (2016).

10. Kasper, J. C. et al. Solar Wind Electrons Alphas and Protons (SWEAP) investigation: design of the solar wind and coronal plasma instrument suite for Solar Probe Plus. Space Sci. Rev. 204, 131-186 (2016).

11. Bale, S. D. et al. The FIELDS instrument suite for Solar Probe Plus. Measuring the coronal plasma and magnetic field, plasma waves and turbulence, and radio signatures of solar transients. Space Sci. Rev. 204, 49-82 (2016).

12. Schatzman, E. A theory of the role of magnetic activity during star formation. Ann. d'Astr. 25, 18-29 (1962)

13. Weber, E. J. \& Davis, L. Jr The angular momentum of the solar wind. Astrophys. J. 148, 217-227 (1967).

14. Finley, A. J., Matt, S. P. \& See, V. The effect of magnetic variability on stellar angular momentum loss. I. The solar wind torque during sunspot cycles 23 and 24 . Astrophys. J. $\mathbf{8 6 4}, 125$ (2018).

15. Bale, S. D. et al. Highly structured slow solar wind emerging from an equatorial coronal hole. Nature https://doi.org/10.1038/s41586-019-1818-7 (2019).

16. Elliott, H. A., Henney, C. J., McComas, D. J., Smith, C. W. \& Vasquez, B. J. Temporal and radial variation of the solar wind temperature-speed relationship. J. Geophys. Res. Space Phys. 117, A09102 (2012).

17. Pilipp, W. G. et al. Characteristics of electron velocity distribution functions in the solar wind derived from the helios plasma experiment. J. Geophys. Res. Space Phys. 92, 1075-1092 (1987).

18. McComas, D. M. et al. Probing the energetic particle environment near the Sun. Nature https://doi.org/10.1038/s41586-019-1811-1 (2019).

19. Vasquez, B. J. \& Hollweg, J. V. Formation of arc-shaped Alfvén waves and rotational discontinuities from oblique linearly polarized wave trains. J. Geophys. Res. 101, 13527-13540 (1996).

20. Bruno, R. \& Carbone, V. The solar wind as a turbulence laboratory. Living Rev. Sol. Phys. 10, 2 (2013).

21. Richardson, I. G. Solar wind stream interaction regions throughout the heliosphere. Living Rev. Sol. Phys. 15, 1(2018).

22. Axford, W. I. The solar wind. Sol. Phys. 100, 575-586 (1985).

Publisher's note Springer Nature remains neutral with regard to jurisdictional claims in published maps and institutional affiliations.

(c) The Author(s), under exclusive licence to Springer Nature Limited 2019 


\section{Methods}

\section{Data collection and analysis}

The data presented here were collected over the course of the first two encounters of the Sun by PSP in November 2018 and April 2019. This study makes use of all of the in situ instruments on the spacecraft. The thermal plasma properties are measured by the PSP SWEAP (Solar Wind Electrons Alphas and Protons) instrument suite ${ }^{10}$, including the Solar Probe Cup (SPC) and the Solar Probe Analyzers (SPAN) for electron and ion plasma data. Magnetic-field data from the outboard FIELDS magnetometer were also used ${ }^{11,15}$, along with energetic-particle rates as seen by IS $\odot \mathrm{IS}^{18}$.SPC measures the reduced distribution function of ionized hydrogen and helium and the two-dimensional flow angles of the ions as a function of energy/charge. These measurements were performed at least once per second and typically more than four times per second throughout the encounter phase of each orbit (below $0.25 \mathrm{AU}$ or $54 R_{\odot}$ ). This paper uses moments of the entire SPC proton distribution function to calculate the total effective proton velocity, density and radial component of the temperature. While the SPAN ion sensor generally did not view the peak of the proton velocity distribution, the overlapping regions seen by SPAN and SPC were compared to confirm that there were no gross offsets in the calibration or the derived plasma properties such as the velocity; this technique will be more accurate when the solar wind flows into SPAN closer to the Sun. Observations of electrons with a central energy of $314 \mathrm{eV}$ and a width of $22 \mathrm{eV}$ by the two SPAN electron sensors were combined, along with the FIELDS determination of the magnetic-field direction, to create the electron pitch-angle distributions.

All data are being archived and will be available for download at the NASA Space Physics Data Facility in November 2019 (https:// spdf.gsfc.nasa.gov/). Additional SWEAP data and information are available at the SWEAP web page (http://sweap.cfa.harvard.edu/). Data were analysed and graphics were developed in IDL (Interactive Data Language).

\section{Statistics}

The distributions of plasma properties in Fig. 1 and Extended Data Fig. 1 were produced with a time resolution of $1 \mathrm{~h}$. During the encounters, the time resolution of the plasma instrument ranged from slightly more than one measurement per second to more than four measurements per second, so each column represents the distribution of approximately 3,600-14,400 measurements. All error bars indicate one standard deviation of the measurements from the mean. At least $10,000-$ and generally more than $80,000-$ observations were used to calculate the mean transverse flow $V_{\mathrm{pT}}$ in Fig. 4.

\section{Estimates of uncertainty}

Here we discuss the absolute accuracy of SPC ion measurements. As verified by ground testing, the absolute accuracy for $V_{\mathrm{pR}}$ is less than $0.01 \%$ over a measurable range of approximately $119 \mathrm{~km} \mathrm{~s}^{-1}$ to $1,065 \mathrm{~km} \mathrm{~s}^{-1}$. The absolute accuracy in temperature is similarly small over a measurable range of approximately $7.3 \mathrm{kK}$ to $21.1 \mathrm{MK}$ (that is, proton thermal speeds of $11 \mathrm{~km} \mathrm{~s}^{-1}$ to $600 \mathrm{~km} \mathrm{~s}^{-1}$ ). Speeds and temperatures at the extremes of these ranges are subject to systematic considerations, but no such measurements are presented here. The accuracy of the density measurement is determined by comparison with the plasma frequency as observed by FIELDS ${ }^{11}$. Thus, the absolute accuracy of the SPC density measurement is estimated to be about $1 \%$ and is no worse than $3 \%$. The absolute accuracy for off-radial flow components is verified via spacecraft roll manoeuvres about the SPC symmetry axis. For solarwind fluxes typical of the first two encounters, the uncertainty associated with this calibration corresponds to a typical absolute accuracy of about $0.5^{\circ}$. For a solar wind of $400 \mathrm{~km} \mathrm{~s}^{-1}$ this corresponds to an expected error in $V_{\mathrm{pT}}$ of $3-4 \mathrm{~km} \mathrm{~s}^{-1}$, which is much smaller than the net rotational flow observed.

\section{Signatures of Alfvénic fluctuations}

In discussing Fig. 2, we stated that the correlation of fluctuations in the components of $\mathbf{B}$ and $\mathbf{V}$ were generally indicative of outward-propagating Alfvén waves. We consider the vector waves or fluctuations $\Delta \mathbf{V}$ and $\Delta \mathbf{B}$ superimposed on a steady background of $\mathbf{B}_{0}$ and $\mathbf{V}_{0}$, respectively. In the long-wavelength fluid magnetohydrodynamic limit, Alfvén waves propagate exactly in parallel or antiparallel directions to $\mathbf{B}_{0}$, are dispersionless and do not compress the plasma, and there is a simple linear relationship of $\Delta \mathbf{B}= \pm D_{\mathrm{A}} \Delta \mathbf{V}$, where $D_{\mathrm{A}}=\left(n_{\mathrm{p}}+4 n_{\alpha}\right)^{0.5} \Theta / 21.8$ (in units of $\mathrm{nT} \mathrm{km}{ }^{-1} \mathrm{~s}$; densities are in units of $\left.\mathrm{cm}^{-3}\right)$ and $\Theta=\left(1-\beta_{\|}+\beta_{\perp}\right)^{-0.55}$. Here $\Theta$ is a correction for thermal pressure anisotropy, where $\beta_{\|}$is the ratio of the parallel plasma pressure to the magnetic pressure and $\beta_{\perp}$ is the ratio of the perpendicular plasma pressure to the magnetic pressure. For this period we find on average $n_{\mathrm{p}}=220 \mathrm{~cm}^{-3}, \beta_{\|}=0.202$ and $\beta_{\perp}=0.315$. SPC and SPAN were not configured optimally to measure the ionized helium abundance $n_{\alpha}$, so assuming the typical range $0.5 \%<n_{\alpha} / n_{\mathrm{p}}<4.5 \%$, we expect $D_{\mathrm{A}}=0.68-0.74 \mathrm{nT} \mathrm{km}^{-1} \mathrm{~s}$. We find $D_{\mathrm{A}}$ for the R, T, N components to be $0.71,1.09$ and $0.70 \mathrm{nT} \mathrm{km}^{-1} \mathrm{~s}$, respectively, so the R and N components are exactly within the expected range and the fluctuations in the T direction are about $33 \%$ higher (it is typical for $D_{\mathrm{A}}$ to be different for each component of the velocity ${ }^{5}$ ). We then used the calculated value of $D_{\mathrm{A}}$ to rescale the range of the vector components of $\mathbf{B}$, so they should overlap with $\mathbf{V}$ if the fluctuations were purely Alfvénic. The sign of the relation between $\Delta \mathbf{B}$ and $\Delta \mathbf{V}$ is given by the sign of $-\mathbf{k} \cdot \mathbf{B}_{0}$, where $\mathbf{k}$ is the wavevector and gives the direction of propagation, and $\mathbf{B}$ is an average direction of the field over a long time scale. The ambient direction of the magnetic field outside the large-amplitude fluctuations points towards the Sun and the correlations are overwhelmingly positive, meaning that we are seeing outward-propagating waves.

\section{Identification of velocity spikes}

Isolated velocity spikes were identified by looking for all intervals in each encounter in which the orientation of the magnetic field started in the quiet configuration pointed towards the Sun within $30^{\circ}$, rotated more than $45^{\circ}$ away from the quiet configuration for at least $10 \mathrm{~s}$, and then returned to the original direction. Candidate events were then examined manually to identify starting and ending times.

Acknowledgements The SWEAP Investigation and this study are supported by the PSP mission under NASA contract NNNO6AA01C. The SWEAP team expresses its gratitude to the scientists, engineers and administrators who have made this project a success, both within the SWEAP institutions and from NASA and the project team at JHU/APL. J.C.K. acknowledges support from the 2019 Summer School at the Center for Computational Astrophysics, Flatiron Institute. The Flatiron Institute is supported by the Simons Foundation. S.D.B. acknowledges the support of the Leverhulme Trust Visiting Professorship programme. T.S.H. was supported by UK STFC ST/S0003641/1.

Author contributions J.C.K. is the SWEAP Principal Investigator (PI) and led the data analysis and writing of this Article. S.D.B. is the FIELDS PI and a SWEAP Co-Investigator and provided the magnetic-field observations. J.W.B. leads the US group that developed the solar-wind Faraday cup, and provided guidance on identifying Alfvénic fluctuations. M.B. provided a pre-amplifier ASIC used within the SPAN electron instruments. A.W.C. is the SPC instrument scientist and ensured that the instrument met its performance requirements and was calibrated. B.D.G.C. contributed to the theoretical calculations and the writing of the manuscript. D.W.C. managed the effort at UCB. D.G. was the institutional lead at NASA MSFC, responsible for materials testing and calibration of SPC. S.P.G. provided recommendations on measurement requirements to detect instabilities. L.G. provided related solar observations and results. J.S.H. contributed to the analysis of the electron observations and to the writing of the manuscript. G.C.H. provided a time-of-flight ASIC to reduce the size and power of the SPAN ion instrument. T.S.H. participated in the analysis of the Alfvénic spikes. Q.H. identified magnetic flux ropes. K.G.K. contributed to the writing of the manuscript and provided warm-plasma growth rate calculations. K.E.K. led the SWEAP Science Operations Center and coordinated observing plans between the instruments and the project. M.V. contributed to the writing of the manuscript and the discussion on the relationship between Alfvénic fluctuations and angular momentum. D.E.L. is the institutional lead at Berkeley, responsible for the implementation of the SPAN instruments and the SWEAP Electronics Module suite-wide computer. R.L. is the SPAN ion instrument scientist. B.L. identified flux ropes and other signatures of coronal mass ejections in the data. P.L. coordinated solar furnace testing of the SPC materials before launch. M. Maksimovic peformed the absolute calibration of the density measurements. M. Martinovic evaluated the quality of the velocity distribution functions. N.V.P. carried out numerical 
simulations. J.D.R. contributed to the design of the Faraday cup. R.M.S. helped to interpret the electron pitch-angle distributions. J.T.S. identified potential field rotation causes. M.L.S. provided the overall data pipeline for SWEAP and SPC high-level data products. A.S.

estimated the location of the heliospheric current sheet. P.L.W. set up the SPC calibration at MSFC and then became SPAN electron instrument scientist at Berkeley. K.H.W. arranged the SPC calibration at MSFC. G.P.Z. leads the SWEAP theory team. R.J.M. leads the FIELDS fluxgate magnetometer. D.J.M. is the IS॰IS PI and provided the energetic-particle data

R.L.M. leads the EPI-Lo energetic-particle instrument. M.P. is the FIELDS SOC lead. N.E.R. is the PSP Project Scientist and reviewed jets and similar coronal transients. N.A.S. runs the
IS॰IS Science Operations Center. All authors participated in planning the observations and data collection, reviewed and discussed the observations, and read, provided feedback and approved the contents of the manuscript.

Competing interests The authors declare no competing interests.

Additional information

Correspondence and requests for materials should be addressed to J.C.K.

Reprints and permissions information is available at http://www.nature.com/reprints. 


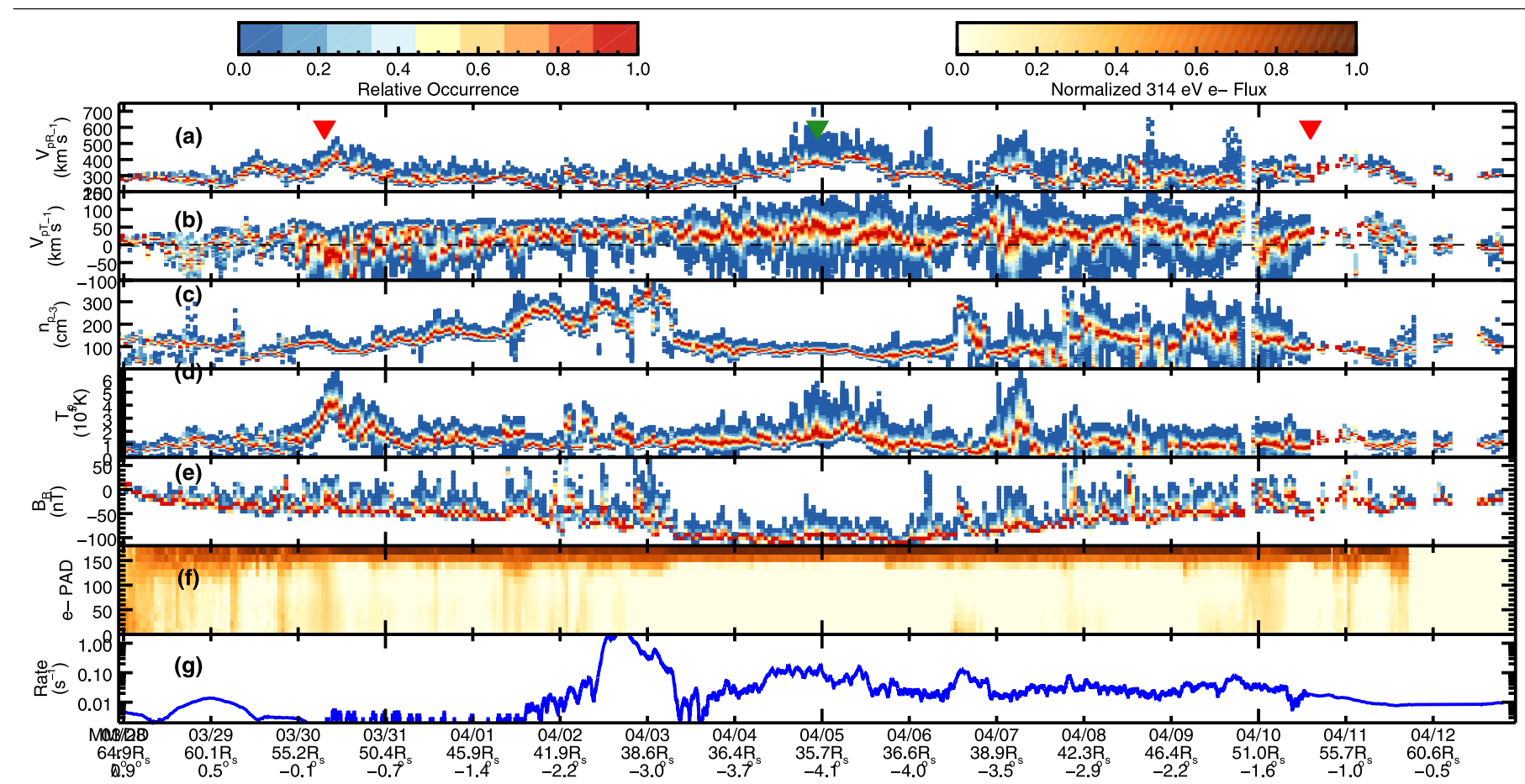

Extended Data Fig. $1 \mid$ Overview of the second PSP encounter with the Sun. The figure is in the same format as Fig. 1. Spikes in the velocity are again seen to

smaller, probably because the Alfvén speed was lower in E2 than E1. The density be coincident with the magnetic-field reversals, but the jump in the speed is 


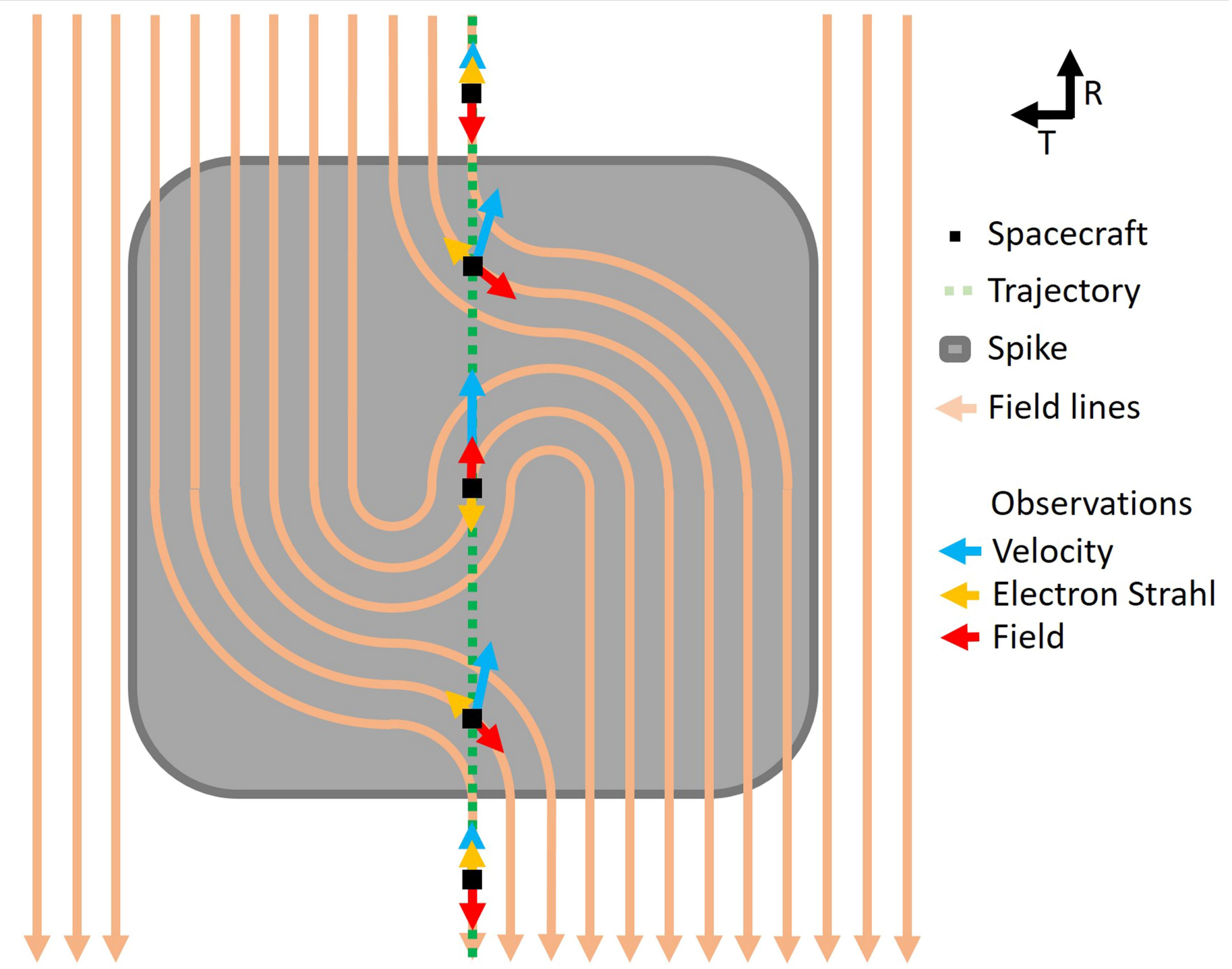

Extended Data Fig. 2 | Schematic of an S-shaped magnetic structure creating a field reversal, heat-flux reversal and a spike in velocity. This figure illustrates the possible geometry of an S-shaped propagating Alfvénic disturbance (grey box) and how it would appear to the spacecraft (black square) as it flew through the spike on the green trajectory. The pink lines with arrows indicate the configuration of the magnetic field, with all field lines ultimately pointing back to the Sun. Arrows at each black square indicate the vector velocity (blue), electron strahl (orange) and magnetic field (red) seen by the spacecraft. If this was a purely Alfvénic structure, then the spike would move away from the Sun in an antiparallel direction to $\mathbf{B}$ at the local Alfvén speed, $C_{\mathrm{A}}$. In the frame of the spike, the shape of the structure would be static, with plasma flowing in along field lines on the upper left and through the spike and emerging at the lower right, always flowing at $C_{\mathrm{A}}$. In the frame of the spacecraft, the constant flow along field lines in the propagating spike frame would translate into a radial increase of $V$ by $C_{\mathrm{A}}$ when $\mathrm{B}$ is perpendicular to the $\mathrm{R}$ direction, and a maximum jump of $2 C_{A}$ when $B$ is completely inverted. Because the heat flux flows away from the Sun along magnetic field lines, it would rotate so as to always be antiparallel to $B$ and appear locally to be flowing back to the Sun at the centre of this disturbance. 


\section{Highly structured slow solar wind emerging from an equatorial coronal hole}

https://doi.org/10.1038/s41586-019-1818-7

Received: 16 July 2019

Accepted: 11 November 2019

Published online: 4 December 2019

\author{
S. D. Bale ${ }^{1,2,3,4 *}$, S. T. Badman, ${ }^{1,2}$, J. W. Bonnell', T. A. Bowen', D. Burgess ${ }^{4}$, A. W. Case ${ }^{5}$, \\ C. A. Cattell ${ }^{6}$, B. D. G. Chandran ${ }^{7,8}$, C. C. Chaston', C. H. K. Chen ${ }^{4}$, J. F. Drake ${ }^{9,10,11}$, \\ T. Dudok de Wit ${ }^{12}$, J. P. Eastwood ${ }^{3}$, R. E. Ergun ${ }^{13}$, W. M. Farrell ${ }^{14}$, C. Fong ${ }^{1,2}$, K. Goetz ${ }^{6}$, \\ M. Goldstein ${ }^{15,16}$, K. A. Goodrich', P. R. Harvey', T. S. Horbury ${ }^{3}$, G. G. Howes ${ }^{17}$, J. C. Kasper ${ }^{5,18}$, \\ P. J. Kellogg ${ }^{6}$, J. A. Klimchuk ${ }^{19}$, K. E. Korreck ${ }^{5}$, V. V. Krasnoselskikh ${ }^{12}$, S. Krucker, ${ }^{1,20}$, R. Laker ${ }^{3}$, \\ D. E. Larson', R. J. MacDowall ${ }^{14}$, M. Maksimovic ${ }^{21}$, D. M. Malaspina ${ }^{13}$, J. Martinez-Oliveros', \\ D. J. McComas ${ }^{22}$, N. Meyer-Vernet ${ }^{21}$, M. Moncuquet ${ }^{21}$, F. S. Mozer', T. D. Phan', M. Pulupa', \\ N. E. Raouafi ${ }^{23}$, C. Salem 1 , D. Stansby ${ }^{3}$, M. Stevens ${ }^{5}$, A. Szabo ${ }^{19}$, M. Velli ${ }^{24}$, T. Woolley ${ }^{3}$ \& \\ J.R. Wygant ${ }^{6}$
}

During the solar minimum, when the Sun is at its least active, the solar wind ${ }^{1,2}$ is observed at high latitudes as a predominantly fast (more than 500 kilometres per second), highly Alfvénic rarefied stream of plasma originating from deep within coronal holes. Closer to the ecliptic plane, the solar wind is interspersed with a more variable slow wind ${ }^{3}$ of less than 500 kilometres per second. The precise origins of the slow wind streams are less certain ${ }^{4}$; theories and observations suggest that they may originate at the tips of helmet streamers ${ }^{5,6}$, from interchange reconnection near coronal hole boundaries ${ }^{7,8}$, or within coronal holes with highly diverging magnetic fields $s^{9,10}$. The heating mechanism required to drive the solar wind is also unresolved, although candidate mechanisms include Alfvén-wave turbulence ${ }^{11,12}$, heating by reconnection in nanoflare ${ }^{13}$, ion cyclotron wave heating ${ }^{14}$ and acceleration by thermal gradients ${ }^{1}$. At a distance of one astronomical unit, the wind is mixed and evolved, and therefore much of the diagnostic structure of these sources and processes has been lost. Here we present observations from the Parker Solar Probe ${ }^{15}$ at 36 to 54 solar radii that show evidence of slow Alfvénic solar wind emerging from a small equatorial coronal hole. The measured magnetic field exhibits patches of large, intermittent reversals that are associated with jets of plasma and enhanced Poynting flux and that are interspersed in a smoother and less turbulent flow with a near-radial magnetic field. Furthermore, plasma-wave measurements suggest the existence of electron and ion velocity-space microinstabilities ${ }^{10,16}$ that are associated with plasma heating and thermalization processes. Our measurements suggest that there is an impulsive mechanism associated with solarwind energization and that micro-instabilities play a part in heating, and we provide evidence that low-latitude coronal holes are a key source of the slow solar wind.
The first solar encounter of the Parker Solar Probe (PSP) occurred during the solar minimum. The spacecraft orbit remained within $5^{\circ}$ of the heliographic solar equator and, unlike any previous spacecraft, was corotational with the Sun for two intervals surrounding perihelion. Figure 1 summarizes the radial magnetic field $\left(B_{R}\right.$; in heliocentric RTN coordinates; see Methods) structure observed by the FIELDS experiment ${ }^{17}$ for a six-week time interval centred on perihelion (6 November 2018). Figure 1a shows 1-s cadence measurements of $B_{\mathrm{R}}$ (see Methods) which show the overall $1 / r^{2}$ behaviour expected from simple flux-conservation arguments ${ }^{18}$ as the heliocentric distance of PSP varied along its eccentric orbit. Against this background, dramatic and unexpected rapid polarity reversals where $\delta B_{\mathrm{R}} /|\mathbf{B}|$ is of the order of 1 are superposed $(|\mathbf{B}|$ is the magnitude of the magnetic field). One-hour statistical modes (most probable value; see Methods) of $B_{\mathrm{R}}$ in Fig. $1 \mathrm{~b}$ remove the transient polarity inversions and reveal the large-scale magnetic structure. Time series predictions of $B_{\mathrm{R}}$ generated from the simple, but widely used, Potential Field Source Surface (PFSS) model ${ }^{19-21}$ are shown for comparison in black and green. The implementation of this model and the procedure to connect it to the location of PSP and generate the time series is discussed in the Methods section 'PFSS modelling and connection to PSP'.

\section{Magnetic field structure}

PFSS is a zero-current force-free model of the global solar corona, meaning that it assumes that magnetic pressure dominates over gas 


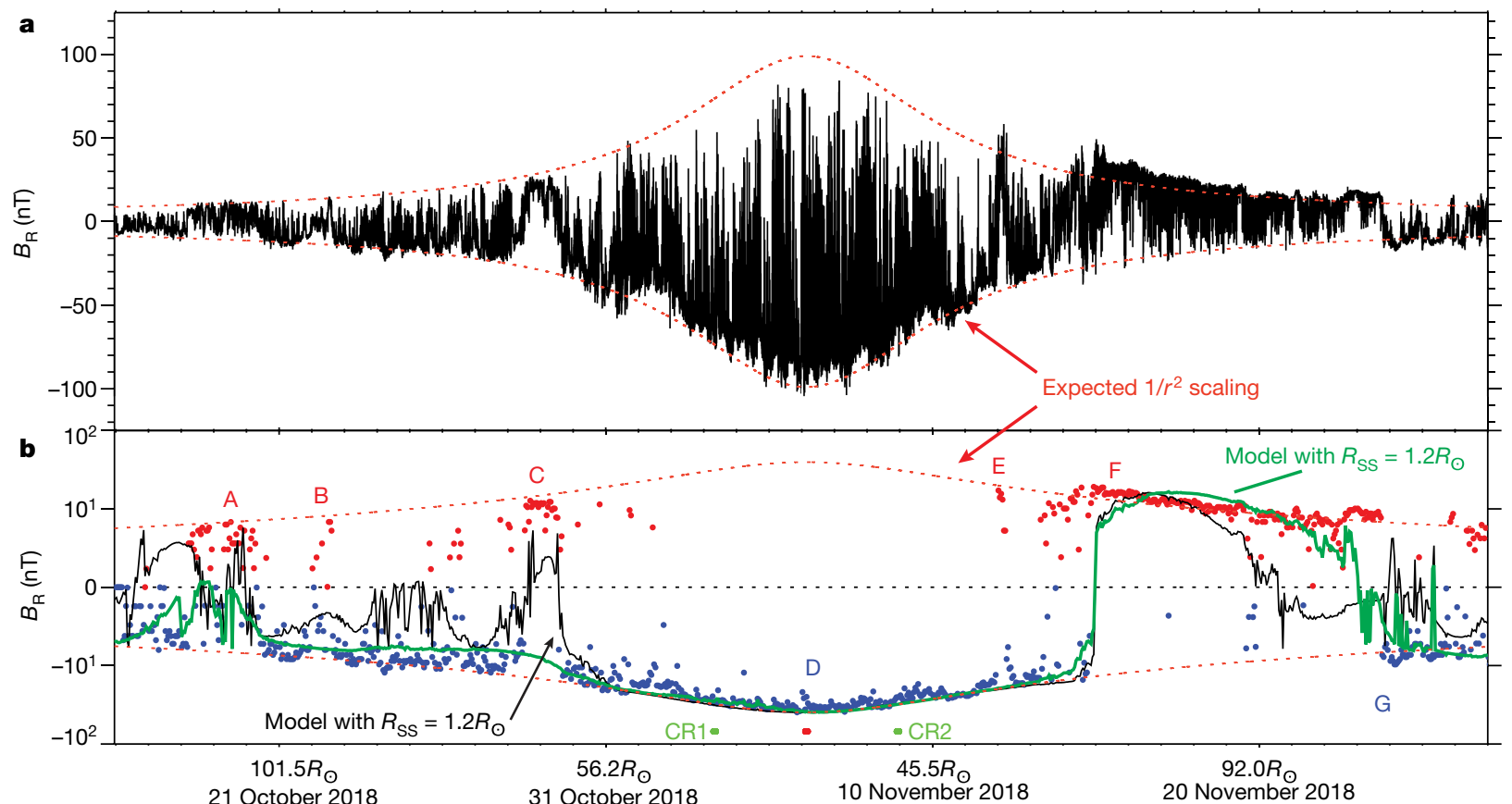

Distance from the Sun Date (UT)

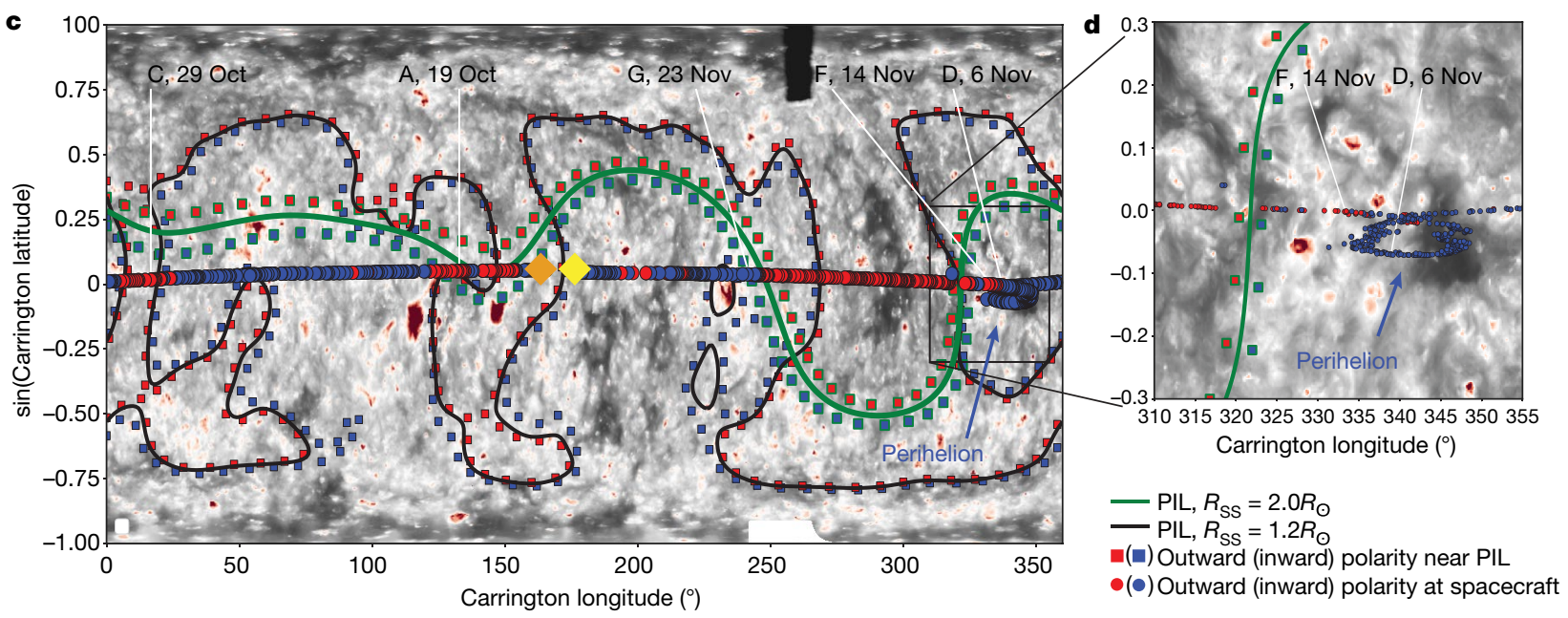

Fig. 1| Radial magnetic field measurements are highly structured, map back to the Sun, and are consistent with a low source surface. $a$, The measured radial magnetic field $B_{\mathrm{R}}$ is comprised of a large-scale field, which scales approximately as $1 / r^{2}$ (red dotted lines; also in Fig. $1 \mathrm{~b}$ ) and rapid largeamplitude polarity reversals $\left(\delta B_{\mathrm{R}} /|\mathbf{B}|\right.$ of the order of 1$)$ associated with jets of plasma (Fig. 2b). b, One-hour statistical modes of $B_{\mathrm{R}}$ (bisymmetric logarithmic plot) show the large-scale radial field coloured for polarity (red, outward; blue inward). Predicted radial-field profiles from a PFSS model are over-plotted using a source-surface radius of $R_{\mathrm{SS}}=1.2 R_{\odot}$ (black curve, unscaled) and $2.0 R_{\odot}$ (green curve, multiplied by a factor of 6.5). $R_{\mathrm{SS}}$ at $1.2 R_{\odot}$ reproduces many of the measured polarity changes (labelled $\mathrm{A}, \mathrm{C}, \mathrm{F}$ and $\mathrm{G}$ ). The $R_{\mathrm{SS}}=2.0 R_{\odot}$ model better predicts the timing of polarity inversion $\mathrm{G}$ (see Methods). Labels $\mathrm{B}$ and $\mathrm{E}$ indicate transient events, and the perihelion coronal hole interval is centred on D. Corotations (CR1 and CR2; green) and the perihelion ( $\mathrm{PH}$; red dot) at $35.7 R_{\odot}$ are labelled. c, An extreme-ultraviolet synoptic map of 171-Å (Fe IX) emission shows structure associated with active regions (small-scale extremeultraviolet bright points appear as red patches) and lower-density plasma in coronal holes (darker regions). The PSP trajectory at the source surface is superimposed, coloured as in $\mathbf{b}$ for measured field polarity. Encounter 1 begins at the orange diamond, moves westwards (in decreasing Carrington longitude, with respect to a fixed point on the solar surface) across the map through perihelion at about $330^{\circ}$, and ends at the yellow diamond. A line shows the location of the model polarity-inversion line (PIL) at the source surface ( $R_{\mathrm{SS}}=1.2 R_{\odot}$, black; $R_{\mathrm{SS}}=2.0 R_{\odot}$, green). Red and blue squares indicate the polarity on either side of the PIL models. $\operatorname{Red}\left(B_{\mathrm{R}}>0\right)$ and blue $\left(B_{\mathrm{R}}<0\right)$ lines map the magnetic field from $R_{\mathrm{SS}}$ back to the photosphere for $R_{\mathrm{SS}}=2.0 R_{\odot}$; for $R_{\mathrm{SS}}=1.2 R_{\odot}$ the model field lines are radial. $\mathrm{d}$, The extreme-ultraviolet map of the perihelion interval, showing field lines mapping back to the Sun into a small equatorial coronal hole, and the location of the adjacent PIL associated with the heliospheric current sheet, from the $2.0 R_{\odot}$ model. pressure (that is, it assumes a low plasma $\beta$ value), so that the problem reduces to magnetostatics, giving a solution of a static field configuration that rigidly corotates with the Sun. The role of gas dynamics is approximated by requiring that the tangential magnetic field vanishes at a spherical 'source surface' at some radius $R_{\mathrm{SS}}$, which simulates how the outflowing solar wind drags the field lines out into the heliosphere.
The magnetostatic approximation limits the accuracy and applicability of the model. Nevertheless, PFSS is widely used as a computationally tractable first approximation and forms the basis for more sophisticated models $^{21,22}$. We note that PSP encounter 1 took place very close to solar minimum, with low solar activity, reducing the impact of nonpotential transient events and active regions. 


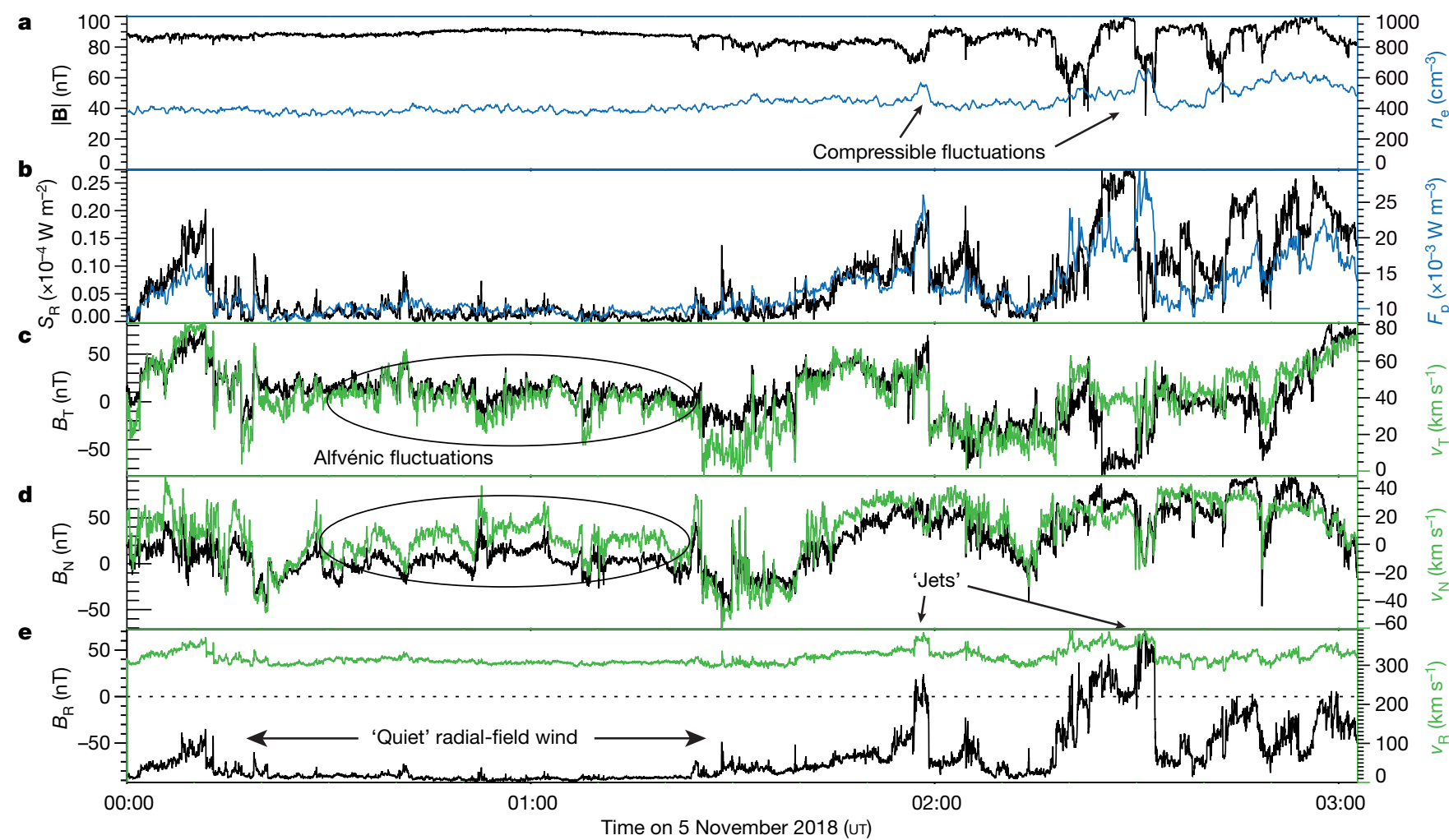

Fig. 2 | Magnetic field reversals and plasma jets carry a Poynting flux. a, Time series measurements of the magnetic field magnitude $|\mathbf{B}|$ (black line) and total plasma density $n_{\mathrm{e}}$ (blue line) show anti-correlation during jet events, consistent with magnetohydrodynamic slow-mode behaviour.b, Radial Poynting flux $\left(S_{\mathrm{R}}\right.$, black line) and ion kinetic energy flux $\left(F_{\mathrm{p}}\right.$, blue line $)$, showing large enhancements during jet-field reversal events. c, Tangential components of the magnetic field $\left(B_{\mathrm{T}} ;\right.$ black $)$ and plasma velocity $\left(v_{\mathrm{T}}\right.$, green) components showing Alfvénic fluctuations.d, The normal components of the magnetic field ( $B_{\mathrm{N}}$, black line) and the plasma velocity $\left(v_{N}\right.$, green line). e, Radial magnetic field $\left(B_{R}\right.$, black line) and plasma velocity $\left(v_{R}\right.$, green line), showing an interval of quiet radial-field wind and flow adjacent to a magnetic structure associated with jets of plasma. Measurements are made from around 00:00 to 03:00 5 November 52018 universal time (UT) at about $36.6 R_{\odot}$. The Alfvén speed during the quiet interval is $v_{\mathrm{A}} \approx 100 \mathrm{~km} \mathrm{~s}^{-1}$.
In Fig. 1b, two model evaluations are shown with $R_{\mathrm{SS}}=2.0 R_{\odot}\left(R_{\odot}\right.$, solar radius; green line) and $R_{\mathrm{SS}}=1.2 R_{\odot}$ (black line). In both cases $R_{\mathrm{SS}}$ is well below the established value ${ }^{23}$. However, this value is necessary to provide good agreement for all model inputs (see Methods) and is not without precedent ${ }^{24,25}$. Model comparison reveals an overall very good agreement for both models, but also shows that the polarity inversions at features $A$ and $C$ are washed out except with the lower source-surface radius (black line). Meanwhile, the timing of feature $G$ is better captured with the higher source-surface radius (green line), illustrating the difficulty that PFSS has with assuming a single source-surface radius, and supports previous findings of a varying 'true' source-surface radius ${ }^{25,26}$. Finally, Fig. 1c, d depicts field-line mappings derived from the same PFSS models shown in Fig. $1 \mathrm{~b}$ to connect the spacecraft down to the lower corona to establish context for the in situ measurements. The spacecraft trajectory is shown projected onto the source surface, coloured by its measured polarity.

The background is a synoptic map of extreme-ultraviolet emission in the 171-Å wavelength for which dark regions imply lower-density plasma and the likely location of open magnetic field lines. For reference, this background is shown in isolation in Extended Data Fig. 4 along with its corresponding map for the 193 -Å wavelength. The neutral lines derived from the PFSS models are shown as single contours in the same colour as their time series in Fig. 1b. Figure 1c shows how the neutral line topology explains the polarity inversions measured by PSP. Figure 1d is a magnification of the two-week interval closest to perihelion $\left(330^{\circ}\right.$ longitude). During the entire two-week corotation loop period, PSP remained connected to a small, negative-polarity, isolated equatorial coronal hole, suggesting that the rapid magnetic field polarity reversals seen in Fig. 1a are magnetic structures emerging from this coronal hole and sweeping past the PSP spacecraft. Extended Data Fig. 5 shows the configuration schematically. For most of this interval, SWEAP ${ }^{27}$ measurements of the solar-wind velocity indicated an Alfvénic slow wind stream (see Fig. 2), suggesting a slow solar wind source rooted in an equatorial coronal hole at the Sun. Polarity inversions $B$ and $E$ in Fig. $1 b$ are associated with (transient) flux rope and coronal mass ejection ${ }^{28}$ events, respectively.

\section{Alfvenic fluctuations and plasma jets}

Time series magnetic field and velocity structures show the correlations (Fig. 2c-e) expected of propagating Alfvén waves ${ }^{29}$, especially during the quiet radial-field intervals. The $\delta B_{\mathrm{R}}$ polarity reversal intervals show enhanced radial wind velocity $v_{\mathrm{R}}$ (Fig. 2e) and the Alfvénic correlations of velocity and magnetic field ( $\delta \mathbf{v}$ to $\delta \mathbf{B})$ within the polarity inversions and jets suggest that these structures may be interpreted as largeamplitude, three-dimensional Alfvénic structures convected away from the Sun. As a simple measure, statistics of zero-crossings (polarity reversals; see Methods) show that around $6 \%$ of the temporal duration of encounter 1 is comprised of these so-defined jets. Many jet intervals show signatures of compressibility (Fig. 2a)-in this case anti-correlated plasma density $n_{\mathrm{e}}$ and $|\mathbf{B}|-$-suggesting slow-mode or pressure-balanced behaviour $^{30}$. Isolated Alfvénic features associated with magnetic field reversals have been identified at $60 R_{\odot}\left(\right.$ ref. $^{31}$ ), near one astronomical unit $(\mathrm{AU})^{32}$ and in the polar heliosphere by the spacecraft Ulysses $^{33}$; however, at those distances few or no compressive signatures were present. It has been suggested ${ }^{34}$ that these magnetic structures could be signatures of impulsive reconnection events in the Sun's atmosphere ${ }^{35}$; simulations ${ }^{36}$ show qualitative similarities to the events of encounter 1 but do not reproduce the observed magnetic field reversals past $90^{\circ}$. 


\section{Article}

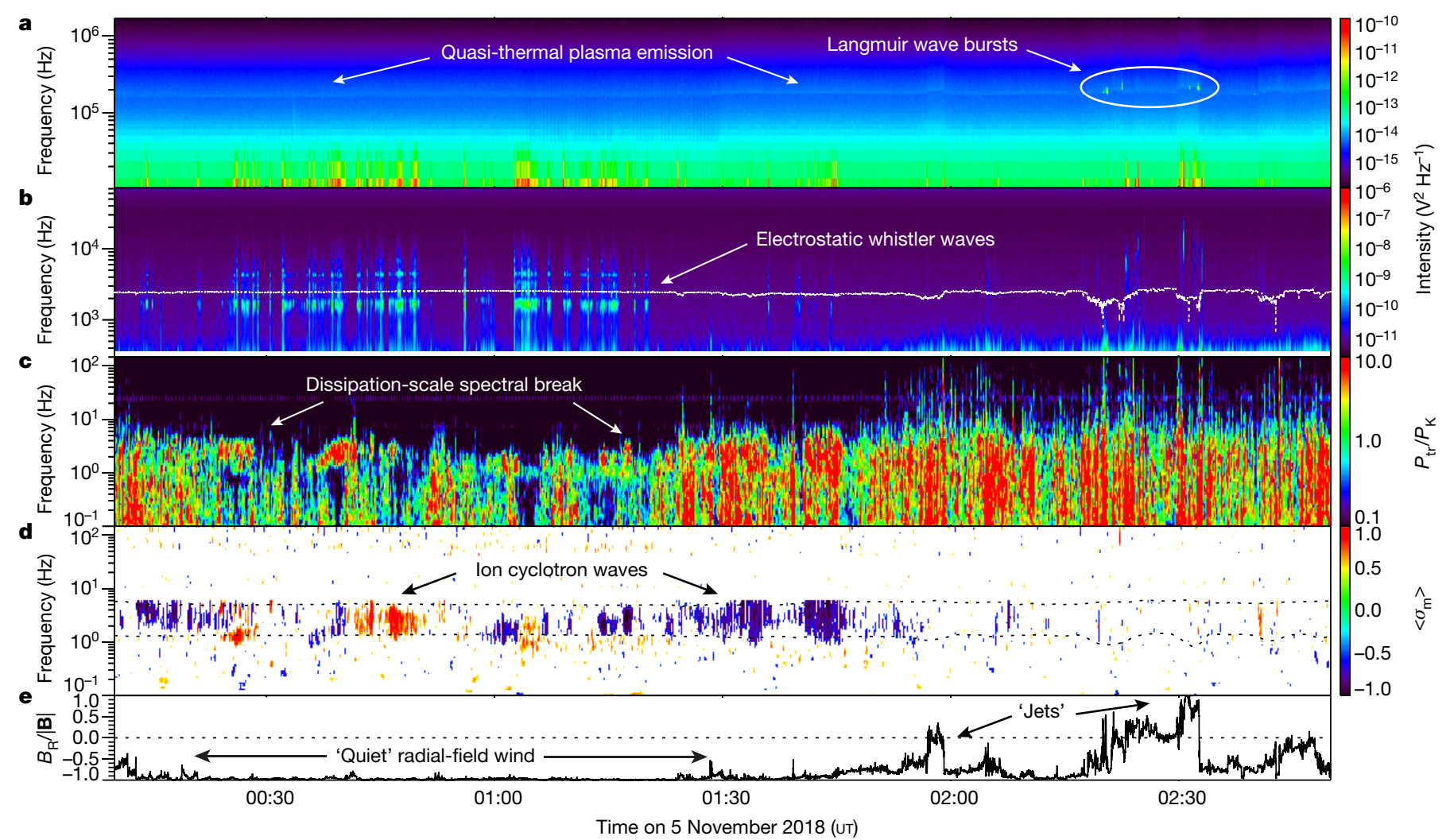

Fig. 3 | Plasma-wave activity near the perihelion differs in quiet wind and jets. a, Spectral density measurements of electric field fluctuations near the electron plasma frequency $f_{\mathrm{pe}}$ show intense bursts of electrostatic Langmuir waves with intensities around $10^{2}-10^{4} \mathrm{~V}^{2} \mathrm{~Hz}^{-1}$ above the thermal background, suggesting the presence of electron beams. b. Electrostatic waves near the electron cyclotron frequency $f_{\text {ce }}$ (white dotted line) and its harmonics are often present in intervals of ambient radial magnetic field, but not present in jet plasma.c, A wavelet spectrogram of the magnetic field shows bursts of turbulent fluctuations with a distinct spectral break between 1 and $10 \mathrm{~Hz}$ associated with a transition to dissipation scales. The trace magnetic

Alfvénic structures and waves have long been considered to be an important energy source for the solar wind ${ }^{11,12}$. The radial Poynting flux $S_{\mathrm{R}}=(\mathbf{E} \times \mathbf{B})_{\mathrm{R}} / \mu_{0}$ (where $\mu_{0}$ is the vacuum permeability and $\mathbf{E}$ is the electric field; see Methods) in the spacecraft frame (Fig. 2b) is about $10 \%$ of the kinetic energy flux (blue curve) and shows enhancements during the jet intervals, suggesting that these plasma jets may impart energy to the emerging solar wind. As seen in Figs. 1a, 2e, the plasma jets appear to be clustered and interspersed in an otherwise quiet solar wind flow with prominently radial magnetic field.

\section{Micro-instabilities and turbulence}

The quiet radial-flow intervals contain plasma waves consistent with expectations of micro-instabilities associated with ion ${ }^{14}$ and electron ${ }^{16}$ velocity-space structure (Fig. 3). The electric field spectrum from about $11 \mathrm{kHz}$ to about $1,688 \mathrm{kHz}$ shows signatures of plasma quasi-thermal noise $^{37}$ (Fig. 3a) at the electron plasma frequency $f_{\mathrm{pe}}$ (which is used to estimate the total plasma density in Fig. 2a). Intense bursts of narrowband electrostatic Langmuir waves (Fig. 3a) occur throughout the perihelion encounter; narrowband Langmuir waves are driven by electron beams and damp rapidly, suggesting the presence of an intermittent, local population of electron beams.

The electric field spectrum (Fig. 3b) from $0.3 \mathrm{kHz}$ to about $75 \mathrm{kHz}$ shows intermittent bursts of electrostatic whistler-wave activity, peaked in power below the electron gyrofrequency $f_{\text {ce }}$. Also present are waves containing harmonic structure consistent with electronBernstein-wave emission. Electrostatic whistler-Bernstein bursts ${ }^{16}$ field intensity $\left(P_{\mathrm{tr}}\right)$ is divided by a factor of $P_{\mathrm{K}} \propto f^{5 / 3}$ such that a power spectrum with index $-5 / 3$ has no frequency dependence. d, Magnetic helicity $\left(\sigma_{m}\right.$, from the wavelet spectrogram) shows narrowband $f_{\mathrm{ci}}<f<f_{\mathrm{ci}}+v_{\mathrm{R}} / v_{\mathrm{A}}$ (the expected Doppler-shifted frequency, where $v_{\mathrm{A}}$ is the local Alfvén speed; dashed lines) signatures associated with ion cyclotron waves - when $\sigma_{\mathrm{m}}$ is close to the maximum (+1) or the minimum (-1)-again in quiet radial solar wind. e, The normalized radial magnetic field $B_{\mathrm{R}} /|\mathbf{B}|$ shows distinct intervals of quiet radialfield wind, reduced turbulent levels and increased occurrence of electrostatic whistler and ion cyclotron instability. Measurements in Fig. 3 were made at approximately 00:00-03:00 November 52018 UT at about $36.6 R_{\odot}$.

are generated by features in the electron velocity distribution function $f_{\mathrm{e}}(v)$ and are not observed in the solar wind at $1 \mathrm{AU}$. Here they occur only in the quiet radial-field intervals. A wavelet spectrogram (divided by a function $P_{\mathrm{K}} \propto f^{-5 / 3}$ ) of the search coil magnetometer and fluxgate magnetometer data in Fig. $3 \mathrm{c}$ shows the spectral content of the magnetic field to around $146 \mathrm{~Hz}$. A spectral break between $1-10 \mathrm{~Hz}$ (in the spacecraft frame) is highly variable and associated with the transition from a magnetohydrodynamic turbulent cascade to dissipation or dispersion ranges at ion kinetic lengthscales ${ }^{38}$. Note that the overall turbulent levels are lower and more intermittent in the quiet radial wind (Figs. 3c, 4a). The spectrum of magnetic helicity ${ }^{39} \sigma_{\mathrm{m}}$ in Fig. $3 \mathrm{~d}$ indicates intervals of large $\left(1>\sigma_{\mathrm{m}}>0.5\right.$, red; $-0.5<\sigma_{\mathrm{m}}<-1$, blue $)$ circular polarization often associated with ion cyclotron waves ${ }^{40}$. These ion wave events are apparent during quiet radial-field intervals.

The (trace) magnetic field spectra (see Methods), averaged over 30 min (Fig. 4a), show broken power-law behaviour, with spectral indices roughly comparable to the $-5 / 3$ and $-8 / 3$ predictions for magnetohydrodynamic and kinetic-scale turbulence ${ }^{38}$, respectively. This suggests that by $36.6 R_{\odot}$, the solar wind has already developed a turbulent cascade, to transport energy from large-scale motions to the microscale, where it can be dissipated. In the radial quiet wind (blue trace), where the turbulence level is substantially lower, an enhancement of wave power near the ion cyclotron frequency is observed. In the active jet wind (black trace), a steep spectrum is seen at the plasma ion inertial and gyroscales, indicating a transition to kinetic range turbulence and possibly the dissipation of turbulent energy to 


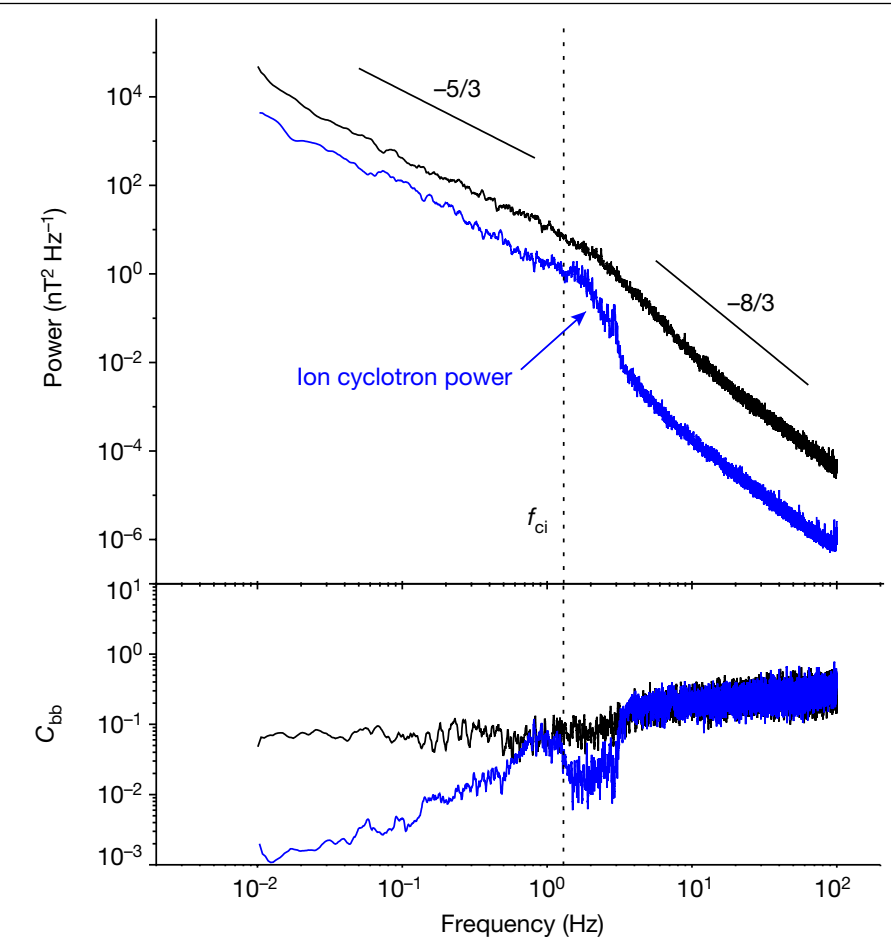

Fig. 4 | Power spectral density and magnetic compressibility of magnetic field fluctuations in quiet and jet wind. a, Thirty-minute integrated power spectra of fluctuations in quiet (blue) and jet (black) solar wind conditions show the transition from a magnetohydrodynamic inertial range to dissipation-or dispersion-range turbulence, here compared to the spacecraftframe frequency $f^{-5 / 3}$ and $f^{8 / 3}$ power laws. The quiet wind spectrum (blue) shows increased power near the ion cyclotron frequency $\left(f_{\mathrm{c}}\right.$; dashed vertical line) that is associated with enhanced magnetic helicity (Fig. 3e). b, The ratio of magnitude $(|\mathbf{B}|)$ to $\operatorname{tr}(\mathbf{B})$ spectra indicates increased magnetic compressibility during jet intervals (black) compared to quiet wind (blue) up to the dissipation scale (a slope of $-8 / 3$ ). The ion cyclotron band corresponds to lower compressibility, as expected.

heat the solar wind as it expands to fill the heliosphere. In both types of wind, the power levels are several orders of magnitude larger than at $1 \mathrm{AU}$. The magnetic compressibility ${ }^{41}$ - defined as $C_{\mathrm{bb}}=(\delta|\mathbf{B}| /|\delta \mathbf{B}|)^{2}$ shows an increase at high frequencies, as expected for kinetic range turbulence (Fig. 4b). At low frequencies, the compressibility is larger in jet wind than in quiet wind, but remains small $\left(C_{\mathrm{bb}}\right.$ less than about 0.1 , indicating that jet fluctuations have an enhanced compressible component but are still predominantly Alfvénic ${ }^{41}$. In the quiet wind, the band of enhanced power near the cyclotron frequency has a reduced magnetic compressibility, as expected for quasi-parallel ion cyclotron waves $^{40}$.

PSP encounter 1 reveals a more structured and dynamic solar wind than is seen at $1 \mathrm{AU}$, with impulsive magnetic field reversals and plasma jets embedded in a quiet radial wind emerging from a small equatorial coronal hole. As PSP goes to lower altitudes, eventually to $9.8 R_{\odot}$ during the upcoming solar maximum, we expect to descend below the the altitude where the wind becomes super-Alfvenic $\left(v_{R}>v_{A}\right)$ and measure the interface between the corona and the solar wind for the first time.

\section{Online content}

Any methods, additional references, Nature Research reporting summaries, source data, extended data, supplementary information, acknowledgements, peer review information; details of author contributions and competing interests; and statements of data and code availability are available at https://doi.org/10.1038/s41586-019-1818-7.
1. Parker, E. N. Dynamics of the interplanetary gas and magnetic fields. Astrophys. J. 128 664-676 (1958).

2. Neugebauer, M. \& Snyder, C. W. Solar plasma experiment. Science 138, 1095-1097 (1962).

3. McComas, D. J. et al. Weaker solar wind from the polar coronal holes and the whole Sun. Geophys. Res. Lett. 35, L18103 (2008).

4. Abbo, L. et al. Slow solar wind: observations and modeling. Space Sci. Rev. 201, 55-108 (2016).

5. Lapenta, G. \& Knoll, D. A. Effect of a converging flow at the streamer cusp on the genesis of the slow solar wind. Astrophys. J. 624, 1049-1056 (2005).

6. Einaudi, G., Boncinelli, P., Dahlburg, R. B. \& Karpen, J. T. Formation of the slow solar wind in a coronal streamer. J. Geophys. Res. 104, 521-534 (1999).

7. Fisk, L. A. \& Schwadron, N. A. The behavior of the open magnetic field of the Sun. Astrophys. J. 560, 425-438 (2001).

8. Antiochos, S. K., Mikic, Z., Titov, V. S., Lionello, R. \& Linker, J. A. A model for the sources of the slow solar wind. Astrophys. J. 731, 112 (2011).

9. Wang, Y.-M. \& Sheeley, N. R. On potential field models of the solar corona. Astrophys. J. 392, 310-319 (1992).

10. Cranmer, S. R. Coronal holes. Living Rev. Sol. Phys. 6, 3 (2009).

11. Hollweg, J. V. \& Johnson, W. Transition region, corona, and solar wind in coronal holes: some two-fluid models. J. Geophys. Res. 93, 9547-9554 (1988).

12. Verdini, A., Velli, M., Matthaeus, W. H., Oughton, S. \& Dmitruk, P. A turbulence-driven model for heating and acceleration of the fast wind in coronal holes. Astrophys. J. Lett. 708, 116-120 (2010).

13. Parker, E. N. Heating solar coronal holes. Astrophys. J. 372, 719-727 (1991).

14. Cranmer, S. R., Field, G. B. \& Kohl, J. L. Spectroscopic constraints on models of ion cyclotron resonance heating in the polar solar corona and high-speed solar wind. Astrophys. J. 518, 937-947 (1999).

15. Fox, N. J. et al. The Solar Probe Plus mission: humanity's first visit to our star. Space Sci. Rev. 204, 7-48 (2016).

16. Breneman, A. W. et al. STEREO and Wind observations of intense cyclotron harmonic waves at the Earth's bow shock and inside the magnetosheath. J. Geophys. Res. Space Phys. 118, 7654-7664 (2013)

17. Bale, S. D. et al. The FIELDS instrument suite for Solar Probe Plus. Space Sci. Rev. 204 49-82 (2016).

18. Parker, E. N. Dynamical theory of the solar wind. Space Sci. Rev. 4, 666-708 (1965).

19. Altschuler, M. D. \& Newkirk, G. Magnetic fields and structure of the solar corona: 1. Methods of calculating coronal fields. Sol. Phys. 9, 131-149 (1969).

20. Schatten, K. H., Wilcox, J. M. \& Ness, N. F. A model of interplanetary and coronal magnetic fields. Sol. Phys. 6, 442-455 (1969).

21. Arge, C. N. \& Pizzo, V. J. Improvement in the prediction of solar wind conditions using near-real time solar magnetic field updates. J. Geophys. Res. Space Phys. 105, 10465-10479 (2000).

22. Riley, P. et al. A comparison between global solar magnetohydrodynamic and potential field source surface model results. Astrophys. J. 653, 1510-1516 (2006).

23. Hoeksema, J. T. Structure and Evolution of the Large Scale Solar and Heliospheric Magnetic Fields. PhD thesis, Stanford Univ. (1984).

24. Lee, C. O. et al. Coronal field opens at lower height during the solar cycles 22 and 23 minimum periods: IMF comparison suggests the source surface should be lowered. Sol. Phys. 269, 367-388 (2011)

25. Riley, P. et al. Predicting the structure of the solar corona and inner heliosphere during Parker Solar Probe's first perihelion pass. Astrophys. J. Lett. 874, 15 (2019).

26. Levine, R. H., Altschuler, M. D., Harvey, J. W. \& Jackson, B. V. Open magnetic structures on the Sun. Astrophys. J. 215, 636-651 (1977).

27. Kasper, J. C. et al. Solar Wind Electrons Alphas and Protons (SWEAP) investigation. Space Sci. Rev. 204, 131 (2016).

28. McComas, D. J. et al. Probing the energetic particle environment near the Sun. Nature https://doi.org/10.1038/s41586-019-1811-1 (2019).

29. Belcher, J. W. \& Davis, L. Large-amplitude Alfvén waves in the interplanetary medium, 2. J. Geophys. Res. 76, 3534-3563 (1971).

30. Howes, G. G. et al. The slow mode nature of compressible wave power in solar wind turbulence. Astrophys. J. Lett. 753, 19 (2012).

31. Horbury, T. S. et al. Short, large-amplitude speed enhancements in the near-Sun fast solar wind. Mon. Not. R. Astron. Soc. 478, 1980-1986 (2018).

32. Gosling, J. T., Tian, H. \& Phan, T. D. Pulsed Alfvén waves in the solar wind. Astrophys. J. Lett. 737, 35 (2011)

33. Balogh, A., Forsyth, R. J., Lucek, E. A., Horbury, T. S. \& Smith, E. J. Heliospheric magnetic field polarity inversions at high heliographic latitudes. Geophys. Res. Lett. 26, 631-634 (1999).

34. Yamauchi, Y., Moore, R. L., Suess, S. T., Wang, H. \& Sakurai, T. The magnetic structure of Ha macrospicules in solar coronal holes. Astrophys. J. 605, 511-520 (2004).

35. Raouafi, N.-E. \& Stenborg, G. Role of transients in the sustainability of solar coronal plumes. Astrophys. J. 787, 118 (2014).

36. Roberts, M. A., Uritsky, V. M., DeVore, C. R. \& Karpen, J. T. Simulated encounters of the Parker Solar Probe with a coronal-hole jet. Astrophys. J. 866, 14 (2018).

37. Meyer-Vernet, N., Issautier, K. \& Moncuquet, M. Quasi-thermal noise spectroscopy: the art and the practice. J. Geophys. Res. Space Phys. 122, 7925-7945 (2017).

38. Schekochihin, A. A. et al. Astrophysical gyrokinetics: kinetic and fluid turbulent cascades in magnetized weakly collisional plasmas. Astrophys. J. 182, 310-377 (2009).

39. Matthaeus, W. H., Goldstein, M. L. \& Smith, C. Evaluation of magnetic helicity in homogeneous turbulence. Phys. Rev. Lett. 48, 1256-1259 (1982).

40. Jian, L. K. et al. Electromagnetic waves near the proton cyclotron frequency: STEREO observations. Astrophys. J. 786, 123 (2014); erratum 847, 1 (2017).

41. Bruno, R. \& Carbone, V. The solar wind as a turbulence laboratory. Living Rev. Sol. Phys. 10, 2 (2013).

Publisher's note Springer Nature remains neutral with regard to jurisdictional claims in published maps and institutional affiliations.

(C) The Author(s), under exclusive licence to Springer Nature Limited 2019 


\section{Article}

${ }^{1}$ Space Sciences Laboratory, University of California, Berkeley, CA, USA. ${ }^{2}$ Physics Department, University of California, Berkeley, CA, USA. ${ }^{3}$ The Blackett Laboratory, Imperial College London, London, UK. ${ }^{4}$ School of Physics and Astronomy, Queen Mary University of London, London, UK. ${ }^{5}$ Smithsonian Astrophysical Observatory, Cambridge, MA, USA. ${ }^{6}$ School of Physics and Astronomy, University of Minnesota, Minneapolis, MN, USA. ${ }^{7}$ Department of Physics \& Astronomy, University of New Hampshire, Durham, NH, USA. ${ }^{8}$ Space Science Center, University of New Hampshire, Durham, NH, USA. ${ }^{9}$ Department of Physics, University of Maryland, College Park, MD, USA. ${ }^{10}$ Institute for Physical Science and Technology, University of Maryland, College Park, MD, USA. "Joint Space Science Institute, University of Maryland College Park, MD, USA. ${ }^{12} \mathrm{LPC} 2 \mathrm{E}$, University of Orléans, CNRS, Orléans, France. ${ }^{13}$ Laboratory for Atmospheric and Space Physics, University of Colorado, Boulder, CO, USA. ${ }^{14}$ Code 695 , NASA
Goddard Space Flight Center, Greenbelt, MD, USA. ${ }^{15}$ Goddard Planetary Heliophysics Institute, University of Maryland Baltimore County, Baltimore, MD, USA. ${ }^{16}$ Code 672 , NASA Goddard Space Flight Center, Greenbelt, MD, USA. ${ }^{17}$ Department of Physics and Astronomy, University of lowa, lowa City, IA, USA.${ }^{18} \mathrm{Climate}$ and Space Sciences and Engineering, University of Michigan, Ann Arbor, MI, USA. ${ }^{19}$ Heliophysics Division, NASA Goddard Space Flight Center, Greenbelt, MD, USA. ${ }^{20}$ University of Applied Sciences and Arts Northwestern Switzerland, Windisch, Switzerland. ${ }^{21}$ LESIA, Observatoire de Paris, Université PSL, Sorbonne Université, CNRS, Meudon, France. ${ }^{22}$ Department of Astrophysical Sciences, Princeton University, Princeton, NJ, USA. ${ }^{23}$ Johns Hopkins University Applied Physics Laboratory, Laurel, MD, USA. ${ }^{24}$ Department of Earth, Planetary, and Space Sciences, University of California, Los Angeles, CA, USA. *e-mail: bale@berkeley.edu 


\section{Methods}

\section{Heliocentric RTN coordinates}

We use so-called heliocentric RTN coordinates in our study. $R$ points from the Sun centre to the spacecraft. $T$ lies in the spacecraft plane (close to the ecliptic) and is defined as the cross product of the solar rotation axis with $\mathrm{R}$. T points in the direction of prograde rotation. $\mathrm{N}$ completes a right-handed system.

\section{Statistical modes}

To examine the large-scale magnetic structure (Fig. 1b), we seek to remove the rapidly varying spikes observed in Fig. 1a. To do this we produce statistical modes which are defined by binning the full cadence magnetic field observations into one-hour intervals and for each interval, calculating the modal value-the peak of the histogram of field values within each interval.

\section{Identification of jet intervals}

We calculate that approximately $6 \%$ of the duration of encounter 1 consists of jet intervals. That number is computed by measuring the duration of positive polarity $B_{\mathrm{R}}$ intervals $(58,973 \mathrm{~s})$ occurring from 30 October 2018 to 11 November 2018 uT $(103,6800$ s total). This interval was chosen to correspond to the negative polarity interval centred on D (Fig. 1b), which has primarily negative polarity over the coronal hole, and does not have transient coronal mass ejection events. The positivepolarity jets were identified using a simple zero-crossing algorithm applied to 1-s cadence radial magnetic field data $B_{\mathrm{R}}$. Of course, not all so-called jets contain full polarity reversals. Biasing this calculation with an amplitude offset will produce a larger fraction of jet times; this is an ongoing study.

\section{PSP/FIELDS measurement details}

Measurements presented here were made by the FIELDS ${ }^{17}$ and SWEAP ${ }^{27}$ instruments on the PSP spacecraft. Magnetic field measurements in Fig. 1a were made by the FIELDS fluxgate magnetometer and are averaged to 1-s cadence from their native cadence, which varies from about 2.3 to 293 samples per second over encounter 1 . The $B_{\mathrm{R}}$ data shown in Fig. $1 \mathrm{~b}$ are derived from the 1-s data by computing the distribution of amplitudes in one-hour intervals with an amplitude resolution of $1 \mathrm{nT}$, and by finding the peak value of that distribution: that is, the statistical mode. This technique removes the fluctuating 'jet' intervals, without introducing the amplitude bias of an averaging algorithm.

The magnetic field measurements in Fig. 2 start at 1-s cadence, averaged down from their native cadence as described above. All magnetic field measurements here are calibrated accurate to better than $0.5 \mathrm{nT}$. SWEAP velocity measurements are made by the Solar Probe Cup (SPC) sensor at a cadence of about 1 measurement per $0.87 \mathrm{~s}$ and then averaged to 5 -s intervals. The 1-s cadence magnetic field data are then averaged onto these 5 -s time intervals. This reduces fluctuation noise in the SPC data and provides velocity and magnetic field measurements at the same cadence. The plasma density measurements in Fig. 1a are made using the FIELDS Low Frequency Receiver (LFR) ${ }^{42}$, which measures the fluctuating electric field across the V1-V2 antenna pair ${ }^{17}$ and computes the spectral density (also shown in Fig. 3a). The spectral peak is identified and associated with the electron plasma frequency $f_{\mathrm{pe}}$, as described previously ${ }^{37}$. Hence, the frequency of the peak amplitude gives a reliable estimate of the total plasma density. The spectral resolution of the LFR instrument is $\Delta f / f \approx 4 \%$. The plasma frequency $f_{\mathrm{pe}}$ is proportional to $\sqrt{n_{\mathrm{e}}}$, where $n_{\mathrm{e}}$ is the electron (total) density; therefore the resulting uncertainty in the density measurement is $\Delta n / n \approx 2 \Delta f / f \approx 8 \%$. Electric field measurements used to compute the radial Poynting flux in Fig. $2 \mathrm{~b}$ are measured directly as differential voltage pairs ${ }^{43}$ between the V1-V2 and V3-V4 antennas ${ }^{17}$ and are then calibrated to electric field units by comparison to $-\mathbf{v} \times \mathbf{B}$ as computed from the SPC velocity and fluxgate magnetometer data. This enables us to remove spacecraft offset electric fields and compute an effective probeseparation length, a standard technique used to calibrate electric field instrumentation ${ }^{44}$. The electric field measurement is accurate to approximately $1 \mathrm{mV} \mathrm{m}^{-1}$.

Measurements in Fig. 3a show the full spectrum of the RFS/LFR ${ }^{42}$ receiver in spectrogram form, as measured by the V1-V2 antenna pair. Wave intensity in Fig. 3a ranges from about $6 \times 10^{-17}$ to $1.4 \times 10^{-10} \mathrm{~V}^{2} \mathrm{~Hz}^{-1}$ and is represented logarithmically. The spectral bandwidth of the LFR receiver is $\Delta f / f=4.5 \%$ and the cadence of the measurement is $1 \mathrm{spec}$ trum per $7 \mathrm{~s}$. Figure $3 \mathrm{~b}$ shows the electric field spectrogram of differential voltage measurements on the V1-V2 antenna pair from the Digital Fields Board (DFB) subsystem ${ }^{43}$, with intensity in arbitrary logarithmic amplitude units. The spectral resolution of this channel of the DFB is $\Delta f / f \approx \sim 6-12 \%$ and the measurement cadence is 1 spectrum per $5.5 \mathrm{~s}$. Figure $3 \mathrm{c}$ shows the magnetic field spectrogram of search coil magnetometer measurements from $\mathrm{DFB}^{43}$, with intensity in arbitrary logarithmic amplitude units. The wavelet spectrogram in Fig. $3 \mathrm{~d}$ and magnetic helicity spectrum in Fig. 3e were computed using the $w a v_{-}$data routine for the IDL programming language in the SPEDAS ${ }^{45}$ suite of IDL analysis routines. Wave intensity in Fig. $3 \mathrm{c}$ is represented in logarithmic power in arbitrary units and is divided by a factor $P_{\mathrm{K}} \propto f^{-5 / 3}$ (flattened), such that a power spectrum with spectral index $-5 / 3$ has no frequency dependence.

\section{PFSS modelling and connection to PSP}

Modelling the magnetic field time series (Fig. 1b) and tracing field lines from PSP down into the corona (Fig. 1c, d) was performed in two main steps.

(1) PFSS implementation. PFSS ${ }^{9,19,20}$ modelling used the recent opensource Python implementation $p f s s p y^{46,47}$. This code package is freely available online, extremely flexible with regard to changing the input parameters and efficient (a full PFSS solution can be extracted in about 14 s including downloading the magnetogram on demand). Given a magnetogram and source-surface radius $\left(R_{\mathrm{SS}}\right)$ as boundary conditions, the code solves the Laplace equation (equation (1)) for the magnetic scalar potential, $\Phi_{\mathrm{B}}$, and outputs a full three-dimensional magnetic field within the annular volume bounded by the photosphere and the source-surface parameter. The choice of magnetogram data and values of source-surface height depicted in Fig. 1 are discussed in Methods section 'Choice of magnetogram data and source-surface radius for Fig. 1 ' below.

$$
\nabla^{2} \Phi_{\mathrm{B}}(r)=0
$$

(2) Ballistic propagation. The procedure to magnetically connect PSP to a particular location at the outer boundary of the PFSS solution domain follows refs. ${ }^{48-50}$, where the field line intersecting the position of PSP is assumed to follow a Parker spiral ${ }^{1}$ with a curvature determined by the co-temporal solar wind velocity measurement at that position. As discussed in ref. ${ }^{48}$, although at lower radii this approximation is strongly perturbed by both corotational effects and the acceleration of the solar wind, these effects actually shift the coronal longitude by a similar magnitude but in opposite directions, resulting in an estimated error in longitude of less than $10^{\circ}$. This produces a very simple mapping (equation (2)) from spacecraft spherical Carrington coordinates $\left(r_{\mathrm{PSP}}\right.$, $\left.\theta_{\mathrm{PSP}}, \phi_{\mathrm{PSP}}\right)$ to coordinates on the source surface $(r, \theta, \varphi)$, involving the solar sidereal rotation rate, $\Omega_{\mathrm{S}}$, the measured solar wind speed, $v_{\mathrm{R}}$, and the source-surface height $R_{\mathrm{sS}}$.

$$
\left(\begin{array}{c}
r \\
\theta \\
\phi
\end{array}\right)=\left(\begin{array}{c}
R_{\mathrm{SS}} \\
\theta_{\mathrm{PSP}} \\
\phi_{\mathrm{PSP}}+\frac{\Omega_{\mathrm{S}}}{v_{\mathrm{R}}}\left(r_{\mathrm{PSP}}-R_{\mathrm{SS}}\right)
\end{array}\right)
$$




\section{Article}

To generate time series predictions, we first download a magnetogram, choose a source-surface height and generate a PFSS solution using equation (1). We then take the trajectory of PSP and use equation (2) to produce a time series of latitudes and longitudes on the source surface to which PSP was connected (see red and blue trajectories in Fig. 1c, d, Extended Data Figs. 1-3). For each latitude and longitude we obtain a $B_{\mathrm{R}}$ value at the source surface from the PFSS model. Finally, we scale each $B_{\mathrm{R}}$ value by $C\left(R_{\mathrm{SS}} / r_{\mathrm{PSP}}\right)^{2}$ to produce an estimate of $B_{\mathrm{R}}$ at the location of PSP as a function of time. $C$ is an empirically determined constant used to scale the time series prediction to match the peak measured magnetic field. Its value is dependent on the choice of magnetogram but approaches unity as the source-surface radius decreases and more flux is opened to the heliosphere. For the model results shown in Fig. 1, the values of $C$ are $6.7\left(2.0 R_{\odot}\right.$ model $)$ and $1.4\left(1.2 R_{\odot}\right.$ model $)$.

To produce field line traces and generate Fig. $1 c$, $d$, we start with the time series of latitudes and longitudes on the source surface connected to PSP. For each pair of coordinates, we use $p f s s p y$ 's built-in field line tracer. Given the output of the pfsspy model, we supply the sourcesurface latitudes and longitudes and the field line tracer generates a field line which starts from that point and propagates it down to the photosphere. The model also provides a polarity for each field line generated, and we use these to colourize the field lines plotted in Fig. 1c, d.

\section{Choice of magnetogram data and source-surface radius for Fig. 1}

Synoptic maps of the photospheric magnetic field are available from multiple sources, which give variable outputs from the PFSS model. In this work we consider the NSO/GONG zero-point-corrected data product $^{51}, \mathrm{SDO} / \mathrm{HMI}$ vector magnetogram data product ${ }^{52}$, and the DeRosa (LMSAL) modelled magnetogram ${ }^{53}$. GONG has the advantage of being operationally certified for space weather predictions, SDO/ $\mathrm{HMI}$ is space-based and offers better resolution, and the DeRosa model assimilates HMI data and uses a surface flux transport and far-side helioseismological data to simulate photospheric dynamics such as differential rotation.

Additional variation in the outputs of the PFSS model arises from the time evolution of photospheric observations. Synoptic magnetograms are built by many observations of the Sun from Earth as it rotates with an approximate 27 -day period. Typically, only $\pm 60^{\circ}$ longitude about the central meridian (sub-Earth point) are used for each observation (the grey regions in Extended Data Figs. 1-3). Although these maps can be updated with new data as frequently as observations are made, parts of the Sun facing away from Earth cannot be updated until they rotate into view, meaning all synoptic maps consist of a mix of old and new data and evolve in time.

Finally, the model output depends considerably on the choice of the source-surface radius parameter $\left(R_{\mathrm{SS}}\right)$. The inferred structure at the source surface changes as the source surface is lowered; implied structure such as the PIL-the contour of $B_{\mathrm{R}}=0$-becomes more structured and warped. The foot points of open field lines at the photosphere encompass larger areas, increasing the predicted size of coronal holes, and also increasing the total amount (both positive and negative) of magnetic flux crossing the source surface.

Our approach to make robust conclusions is to generate model results for multiple times from all three magnetogram sources for varying source surface radii. Colour maps of $B_{\mathrm{R}}$ at the source surface and the associated PILs are shown in Extended Data Figs.1-3. The majority of models at $2.0 R_{\odot}$ and below predict polarity inversions in the vicinity of $240^{\circ}$ and $310^{\circ}$ longitude at all source-surface radii, with additional polarity inversions around $10^{\circ}$ and $140^{\circ}$ longitude that develop at lower source-surface radii. These features are all consistent with PSP measurements; we emphasize that they are largely independent of the time of observation and the choice of magnetogram source. Although the established ${ }^{23}$ value of $2.5 R_{\odot}$ still gives good results from a GONG evaluation, both the HMI and DeRosa models produce strong disagreement around the time of perihelion. In Fig. $1 \mathrm{~b}-\mathrm{d}$ we show results from the GONG zero-point-corrected map evaluated on 6 November 2018 UT about which our time range of analysis is symmetric. This evaluation shows all the above features and produces good time series agreements. We show source-surface radii of $2.0 R_{\odot}$ and $1.2 R_{\odot}$. These lower source-surface radii do have modern precedent: $2.0 R_{\odot}$ is consistent with previous PFSS modelling done for the same interva ${ }^{25}$, where that radius was chosen to better match the observed extent of coronal holes. Another previous work ${ }^{24}$ investigated the impact of lowering the source surface radius on model results, observing that at solar minimum a lower $\left(<2.0 R_{\odot}\right)$ source-surface radius was required to populate equatorial coronal holes with open field lines and improve their estimates of magnetic field strength at $1 \mathrm{AU}$.

\section{Data availability}

The data used in this study are available at the NASA Space Physics Data Facility (SPDF), https://spdf.gsfc.nasa.gov/index.html.

42. Pulupa, M. et al. The Solar Probe Plus Radio Frequency Spectrometer: measurement requirements, analog design, and digital signal processing. J. Geophys. Res. Space Phys. 122, 2836-2854 (2017)

43. Malaspina, D. M. et al. The Digital Fields Board for the FIELDS instrument suite on the Solar Probe Plus mission: analog and digital signal processing. J. Geophys. Res. Space Phys. 121, 5088-5096 (2016).

44. Mozer, F. S. DC and low-frequency double probe electric field measurements in space. J. Geophys. Res. Space Phys. 121, 10942-10953 (2016).

45. Angelopoulos, V. et al. The Space Physics Environment Data Analysis System (SPEDAS). Space Sci. Rev. 215, 9 (2019).

46. Stansby, D. Dstansby/pfsspy: pfsspy 0.1.2 https://zenodo.org/record/3237053\#.Xcqc1f7SUk (2019)

47. Yeates, A. Antyeates1983/pfss: first release of pfss code https://zenodo.org/ record/1472183\#. XcqdU1f7SUk (2018).

48. Nolte, J. T. \& Roelof, E. C. Large-scale structure of the interplanetary medium. I: High coronal source longitude of the quiet-time solar wind. Sol. Phys. 33, 241-257 (1973).

49. Neugebauer, M., et al. Spatial structure of the solar wind and comparisons with solar data and models. J. Geophys. Res. 103, 14587-14599 (1998).

50. Stansby, D., Horbury, T. S., Wallace, S. \& Arge, C. N. Predicting large-scale coronal structure for Parker Solar Probe using open source software. RNAAS 3, 57 (2019).

51. Clark, R., Harvey, J., Hill, F. \& Toner, C. GONG magnetogram zero-point-correction status. Bull. Am. Astron. Soc. 35, 822 (2003).

52. Hoeksema, J. T. et al. The Helioseismic and Magnetic Imager (HMI) vector magnetic field pipeline: overview and performance. Sol. Phys. 289, 3483-3530 (2014).

53. Schrijver, C. J. \& DeRosa, M. L. Photospheric and heliospheric magnetic fields. Sol. Phys. 212, 165 (2003).

Acknowledgements The FIELDS experiment on the Parker Solar Probe spacecraft was designed and developed under NASA contract NNNO6AA01C. The FIELDS team acknowledges the contributions of the Parker Solar Probe mission operations and spacecraft engineering teams at the Johns Hopkins University Applied Physics Laboratory. S.D.B. acknowledges the support of the Leverhulme Trust Visiting Professorship programme. Contributions from S.T.B. were supported by NASA Headquarters under the NASA Earth and Space Science Fellowship Program grant 80NSSC18K1201. This work uses data obtained by the Global Oscillation Network Group (GONG) programme, managed by the National Solar Observatory, which is operated by AURA, Inc. under a cooperative agreement with the National Science Foundation. The data were acquired by instruments operated by the Big Bear Solar Observatory, High Altitude Observatory, Learmonth Solar Observatory, Udaipur Solar Observatory, Instituto de Astrofísica de Canarias and Cerro Tololo Interamerican Observatory. D.B. was supported by UK STFC grant ST/P000622/1. J.P.E. and T.S.H. were supported by UK STFC grant ST/S000364/1. D.S. was supported by UK STFC grant ST/NO00692/1. C.H.K.C. is supported by STFC Ernest Rutherford Fellowship number ST/NOO3748/2. T.D.d.W. and V.V.K. are supported by CNES.

Author contributions S.D.B. wrote the manuscript with substantial contributions from S.T.B., B.D.G.C., C.H.K.C., T.S.H., M. Maksimovic, T.D.P. and M.V. All authors participated in the data interpretation, and read and commented upon the manuscript. S.D.B. led the FIELDS instrument team with contributions from J.W.B., T.A.B, T.D.d.W., K.G., P.R.H., D.E.L., R.J.M. M. Maksimovic, D.M.M., M.P. and N.E.R.

Competing interests The authors declare no competing interests.

\section{Additional information}

Correspondence and requests for materials should be addressed to S.D.B.

Reprints and permissions information is available at http://www.nature.com/reprints. 

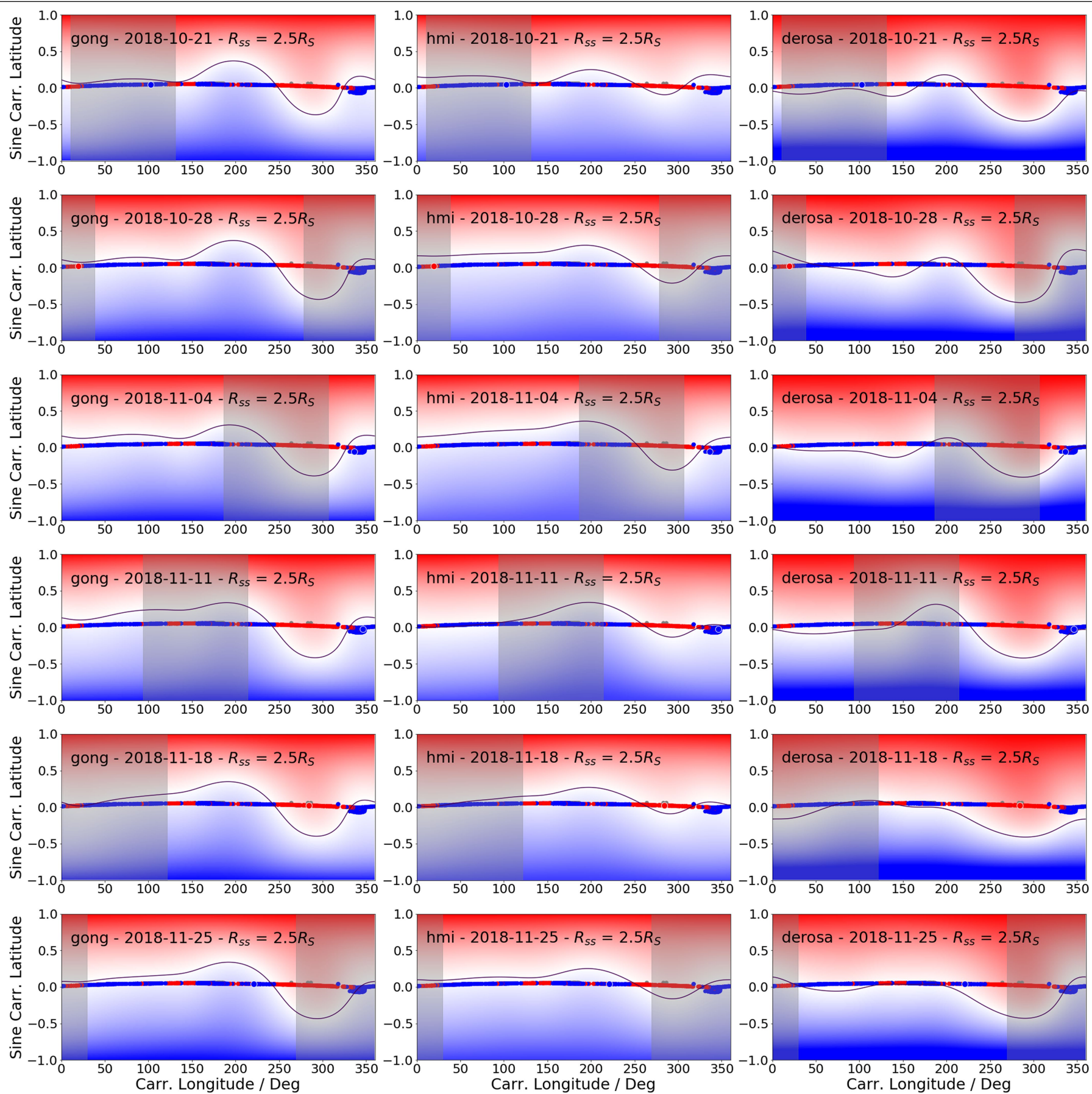

Extended Data Fig. 1 | Variation of PFSS neutral line topology with time and magnetogram choice at $\boldsymbol{R}_{\mathrm{SS}}=\mathbf{2 . 5 \boldsymbol { R } _ { \odot }}$. Colour maps of $B_{\mathrm{R}}$ at the source surface from PFSS extractions with source-surface radius $R_{\mathrm{SS}}=2.5 R_{\odot}$. Red indicates positive polarity and blue indicates negative polarity. The black line shows the PIL (the contour of $B_{\mathrm{R}}=0$ ). Superposed is the ballistically projected PSP trajectory coloured by the measured polarity. Perihelion occurred around $330^{\circ}$ longitude. Left to right, the columns show extractions from the NSO/GONG,
SDO/HMI and DeRosa LMSAL models. From top to bottom, the models are evaluated at a weekly cadence spanning six weeks about perihelion, with input magnetograms from each source taken as close in time as possible. The grey shading shows the region $\pm 60^{\circ}$ about the central meridian on the date of the model evaluation, indicating the portion of the Sun that could be observed at the time of observation. 


\section{Article}
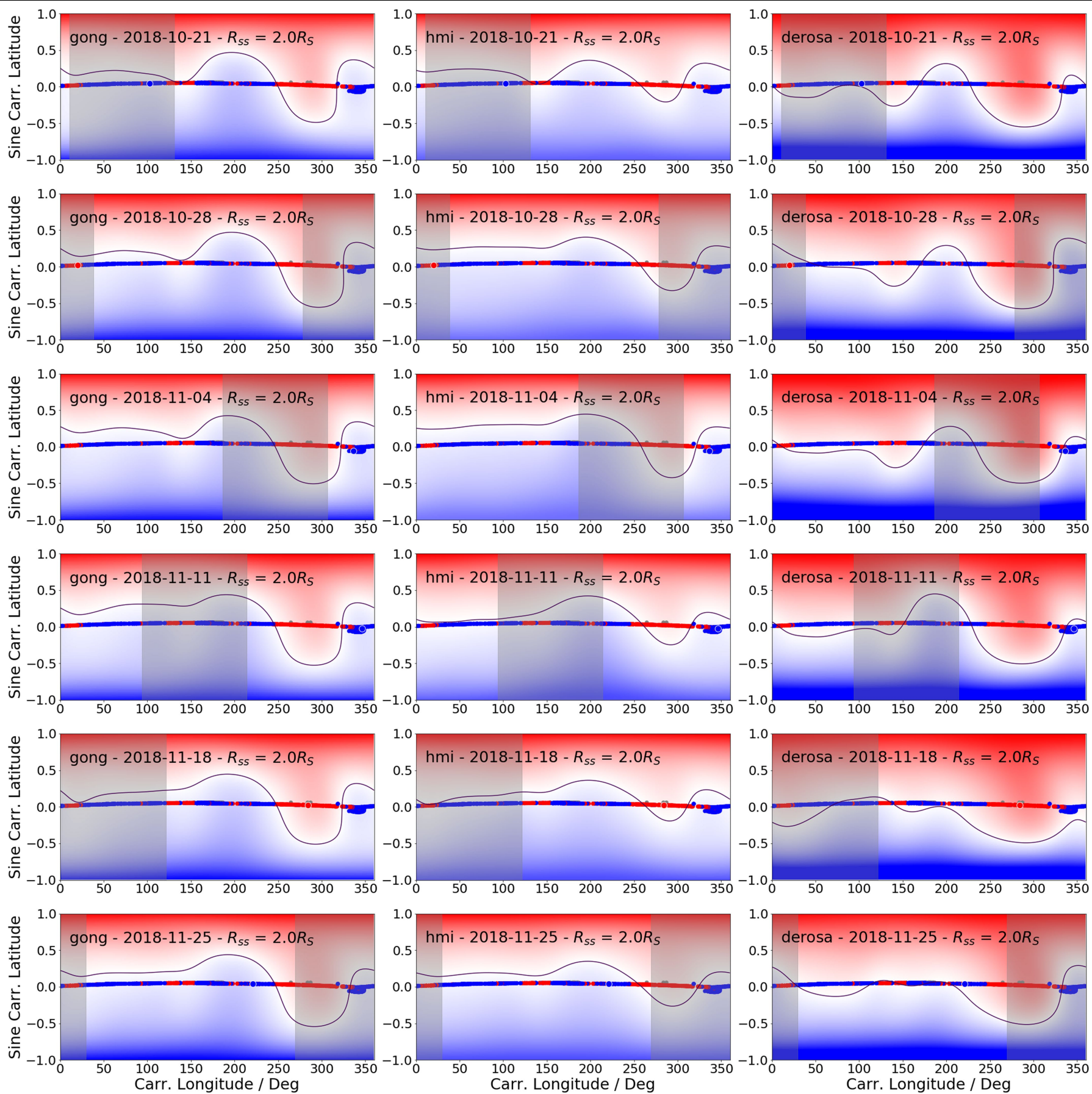

Extended Data Fig. 2 | Variation of PFSS neutral line topology with time and magnetogram choice at $\boldsymbol{R}_{\mathrm{Ss}}=\mathbf{2 . 0 R _ { \odot }}$. Colour maps of $B_{\mathrm{R}}$ at the source surface from PFSS extractions with $R_{\mathrm{SS}}=2.0 R_{\odot}$. Other features are as described in Extended Data Fig. 1 . 

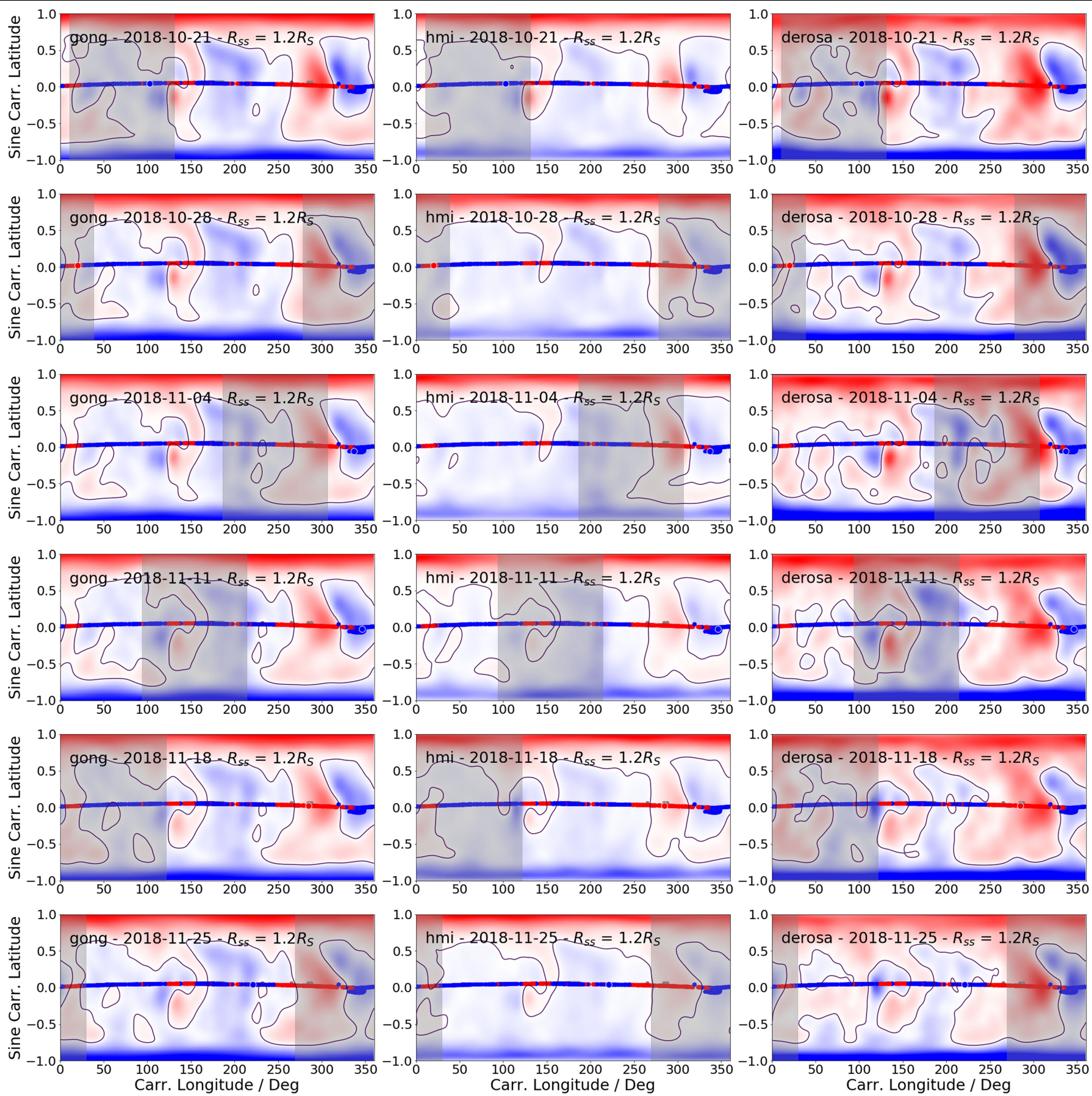

Extended Data Fig. $3 \mid$ Variation of PFSS Neutral Line topology with time and magnetogram choice at $\boldsymbol{R}_{\mathrm{sS}}=1.2 \boldsymbol{R}_{\odot}$. Colour maps of $B_{\mathrm{R}}$ at the source surface from PFSS extractions with $R_{\mathrm{SS}}=1.2 R_{\odot}$. Other features are as described in Extended Data Fig. 1 . 


\section{Article}
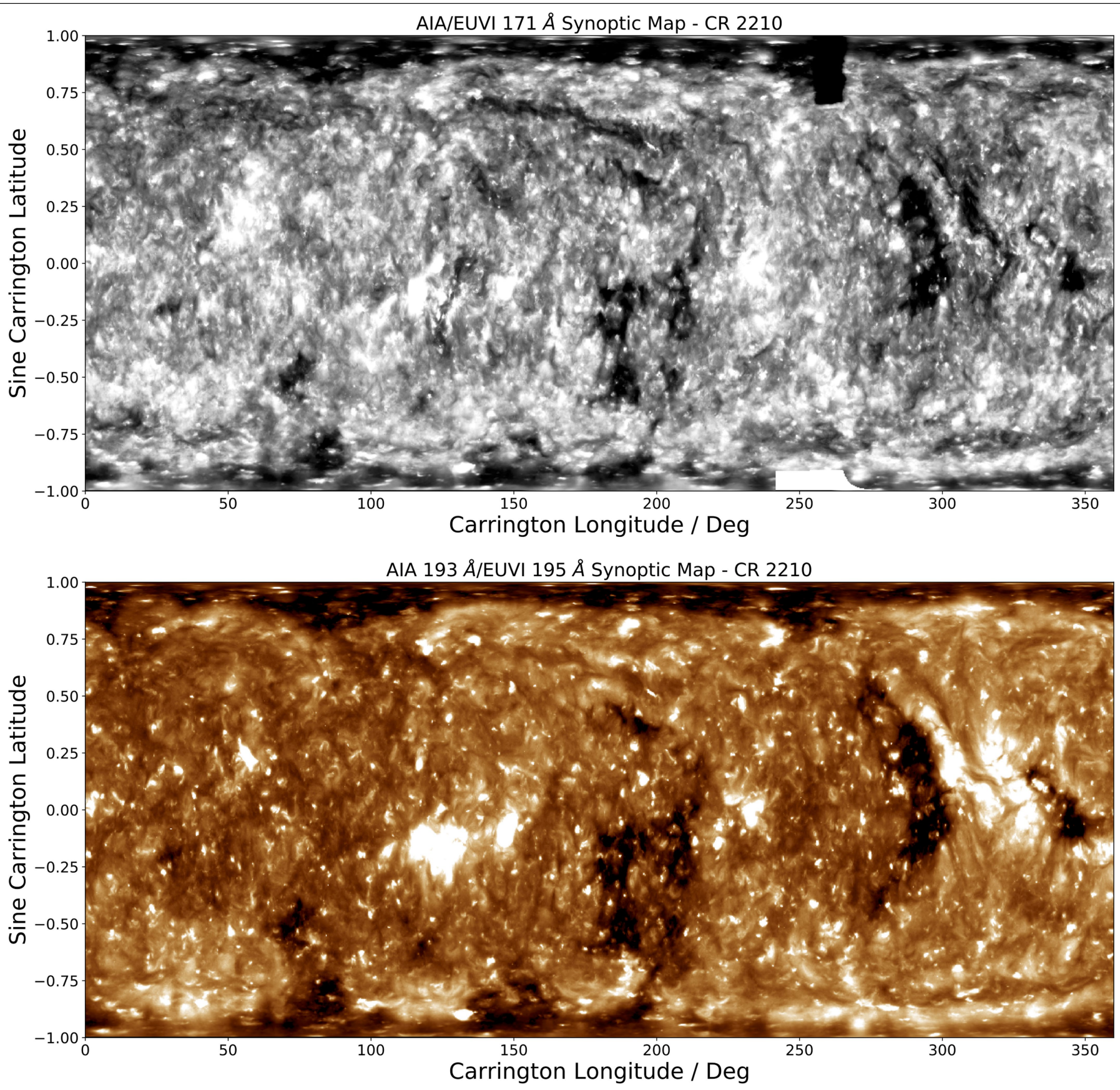

Extended Data Fig. 4 | Synoptic maps of extreme-ultraviolet coronal emission from Carrington rotation 2,210, assembled from the STEREO-A/ EUVI and SDO/AIA instruments. Top, 171-Å data showing coronal Fe IX emission at around $600,000 \mathrm{~K}$. This is the background of Fig. 1c, d. Bottom, 193- $\AA$ (AIA) and 195- $\AA$ (EUVI) data showing emission from coronal Fe XII emission at around $1,000,000 \mathrm{~K}$. The brightness is positively correlated with the integrated plasma density squared along the line of sight. The dark regions in both images are probable locations of coronal holes, which are threaded by open magnetic field lines that allow plasma to evacuate into interplanetary space, resulting in under-dense regions. Carrington rotation 2,210 occurred from 20:5126 October 2018 uT to 04:1123 November 2018 UT. 


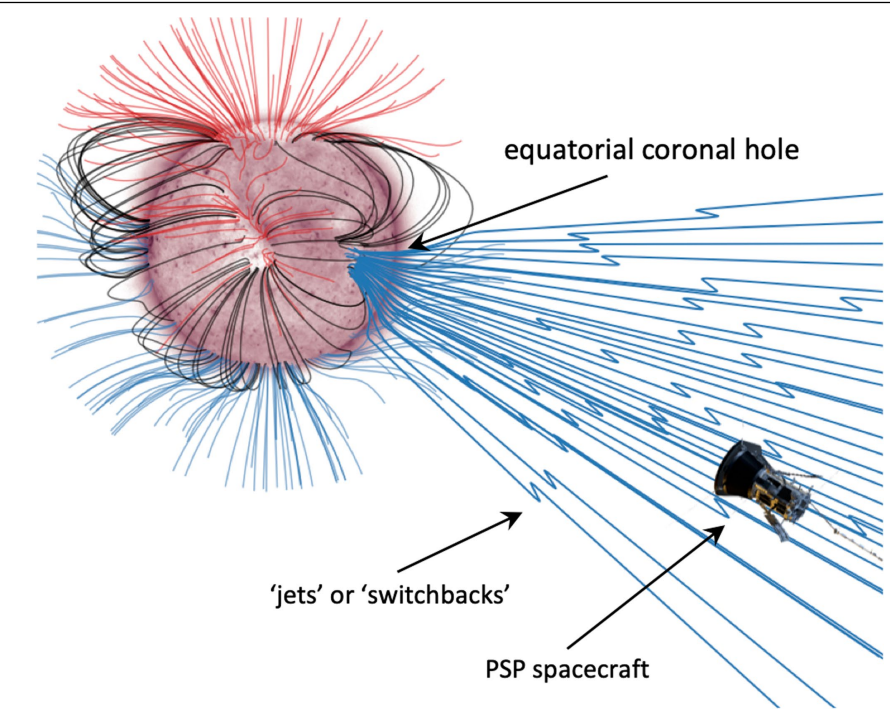

Extended Data Fig. 5 | During encounter 1, PSP connected magnetically to a small negative-polarity equatorial coronal hole. This schematic shows a potential field extrapolation of the solar magnetic field at the time of the first perihelion pass of PSP. The solar surface is shown, coloured by AIA $211-\AA$ extreme-ultraviolet emission (see Extended Data Fig. 4 for other wavelengths). Coronal holes appear as a lighter shade. Superposed are various field lines initialized at the solar disk. Black lines indicate closed loops, blue and red illustrate open field lines with negative and positive polarities, respectively. As depicted here and in Fig. 1c, d, at perihelion PSP connected to a negative equatorial coronal hole. The 'switchbacks' (the jets) observed by PSP (Fig. 1a) are illustrated as kinks in the open field lines emerging from the coronal hole that connect to PSP. (Note that neither the radial distance to the spacecraft nor the scale or amplitude of the jets or switchbacks are to scale.) Spacecraft graphic is courtesy of NASA/Johns Hopkins APL. 